Pacific

Journal of

Mathematics

AN ELEMENTARY CONSTRUCTION OF THE MULTIGRADED HILBERT SCHEME OF POINTS

Mark E. Huibregtse

Volume $223 \quad$ No. 2

February 2006 


\title{
AN ELEMENTARY CONSTRUCTION OF THE MULTIGRADED HILBERT SCHEME OF POINTS
}

\author{
MARK E. Huibregtse
}

\begin{abstract}
We present an elementary construction of the multigraded Hilbert scheme of $d$ points of $A_{k}^{n}=\operatorname{Spec}\left(k\left[x_{1}, \ldots, x_{n}\right]\right)$, where $k$ is an arbitrary commutative and unitary ring. This Hilbert scheme represents the functor from k-schemes to sets that associates to each k-scheme $T$ the set of closed subschemes $Z \subseteq T \times \times_{k} \mathbb{A}_{k}^{n}$ such that the direct image (via the first projection) of the structure sheaf of $Z$ is locally free of rank $d$ on $T$. It is a special case of the general multigraded Hilbert scheme constructed by Haiman and Sturmfels. Our construction proceeds by gluing together affine subschemes representing subfunctors that assign to $T$ the subset of $Z$ such that the direct image of the structure sheaf on $T$ is free with a particular set of $d$ monomials as basis. The coordinate rings of the subschemes representing the subfunctors are concretely described, yielding explicit local charts on the Hilbert scheme.
\end{abstract}

\section{Introduction}

1.1. The multigraded Hilbert scheme of points. The multigraded Hilbert scheme of points is a special case of the multigraded Hilbert scheme introduced by Haiman and Sturmfels [2004]. Let k be an arbitrary commutative and unitary ring, let $S$ be the polynomial ring

$$
S=\mathrm{k}\left[x_{1}, \ldots, x_{n}\right]=\mathrm{k}[\mathbf{x}],
$$

let $\mathbb{A}_{\mathrm{k}}^{n}=\operatorname{Spec}(S)$, and let

$$
H_{\AA_{\mathrm{k}}^{n}}^{d}: \underline{\mathrm{k}-\mathrm{Sch}} \rightarrow \underline{\mathrm{Set}}
$$

be the contravariant functor that assigns to the k-scheme $T$ the set $H_{\AA_{k}^{n}}^{d}(T)$ of subschemes

$$
Z \subseteq T \times{ }_{\mathrm{k}} \mathbb{A}_{\mathrm{k}}^{n}
$$

such that the direct image (via the first projection) of the structure sheaf of $Z$ is locally free of rank $d$ on $T$, and assigns to the map $f: W \rightarrow T$ of k-schemes the

Keywords: Hilbert scheme of points, affine space. 
map

$$
H_{\AA_{k}^{n}}^{d}(f): H_{\AA_{k}^{n}}^{d}(T) \rightarrow H_{\AA_{k}^{n}}^{d}(W), \quad Z \mapsto W \times_{T} Z .
$$

Lemma 1. The subschemes $Z \in H_{\AA_{k}^{n}}^{d}(T)$ are necessarily closed subschemes of $T \times{ }_{\mathrm{k}} \mathbb{A}_{\mathrm{k}}^{n}$.

Proof. Since $\mathbb{A}_{k}^{n}$ is an affine k-scheme, it is separated over $\operatorname{Spec}(\mathrm{k})$; it follows from [Grothendieck and Dieudonné 1971, Prop. 5.3 .1 (iv), p. 279] that the projection $T \times{ }_{\mathrm{k}} \mathbb{A}_{\mathrm{k}}^{n} \rightarrow T$ is separated; moreover, the composite map $Z \subseteq T \times{ }_{\mathrm{k}} \mathbb{A}_{\mathrm{k}}^{n} \rightarrow T$ is universally closed, since $Z$ is finite (hence proper) over $T$. Then [Grothendieck and Dieudonné 1971, Prop. 5.2.8, p. 279] yields that the inclusion $Z \subseteq T \times{ }_{\mathrm{k}} \mathbb{A}_{\mathrm{k}}^{n}$ is universally closed.

The general construction in [Haiman and Sturmfels 2004] implies that the functor $H_{\mathbb{A}_{k}^{n}}^{d}$ is representable by a quasi-projective k-scheme $\mathbf{H}_{\mathrm{A}_{k}^{n}}^{d}$; in other words, $H_{\mathrm{A}_{k}^{n}}^{d}$ is isomorphic to the functor of points of $\mathbf{H}_{\AA_{k}^{n}}^{d}$. In particular, the identity map $\mathbf{H}_{\mathrm{A}_{\mathrm{k}}^{n}}^{d} \rightarrow \mathbf{H}_{\mathrm{A}_{\mathrm{k}}^{n}}^{d}$ corresponds to the universal closed subscheme

$$
Z_{\mathbb{A}_{\mathrm{k}}^{n}}^{d} \subseteq \mathbf{H}_{\mathrm{A}_{\mathrm{k}}^{n}}^{d} \times_{\mathrm{k}} \mathbb{A}_{\mathrm{k}}^{n}
$$

the representability of the functor $H_{\AA_{k}^{n}}^{d}$ can be expressed as the following universal property of the pair $\left(\mathbf{H}_{\mathrm{A}_{\mathrm{k}}^{n}}^{d}, Z_{\mathrm{A}_{\mathrm{k}}^{n}}^{d}\right)$ (see, for example, [Grothendieck and Dieudonné 1971, 0.1.1.8]):

The set of maps $f: T \rightarrow \mathbf{H}_{\mathrm{A}_{k}^{n}}^{d}$ is in natural bijective correspondence with the set of closed subschemes $Z_{f} \subseteq T \times \times_{k} A_{k}^{n}$ such that the direct image of the structure sheaf of $Z_{f}$ is locally free of rank $d$ on $T$; the bijection $f \mapsto Z_{f}$ is defined by $Z_{f}=T \times{ }_{\mathbf{H}_{\mathbb{A}_{k}^{n}}^{d}} Z_{\AA_{k}^{n}}^{d}$.

The goal of this paper is to present an elementary construction of the pair $\left(\mathbf{H}_{\AA_{\mathrm{k}}^{n}}^{d}, Z_{\AA_{\mathrm{k}}^{n}}^{d}\right)$ that uses only polynomial arithmetic - nothing more advanced, such as Grassmannians. It follows easily from our construction that $\mathbf{H}_{\AA_{k}^{n}}^{d}$ is separated and of finite type over $\operatorname{Spec}(k)$, but we do not establish the quasi-projectivity.

Remark 2. Since a k-scheme is determined by the restriction of its functor of points to affine k-schemes (see, for example, [Eisenbud and Harris 2000, Prop. VI-2, p. 253]), the multigraded Hilbert scheme of points $\mathbf{H}_{\AA_{k}^{n}}^{d}$ can be defined as the scheme representing the restriction of the functor $H_{\mathbb{A}_{k}^{n}}^{d}$ to k-algebras $R$, as in [Haiman and Sturmfels 2004]. Precisely, if $R$ is a k-algebra, then

$$
H_{\AA_{\mathrm{k}}^{n}}^{d}(R)=\left\{\begin{array}{l|l}
\text { ideals } I \subseteq R \otimes_{\mathrm{k}} S=R[\mathbf{x}] & \begin{array}{l}
R[\mathbf{x}] / I \text { is locally free of } \\
\operatorname{rank} d \text { over } R
\end{array}
\end{array}\right\} .
$$

In particular, if $\mathrm{k}$ is a field, then $H_{\AA_{\mathrm{k}}^{n}}^{d}(\mathrm{k})$ is the set of ideals $I \subseteq \mathrm{k}\left[x_{1}, \ldots, x_{n}\right]$ such that

$$
\operatorname{dim}_{\mathrm{k}}\left(\mathrm{k}\left[x_{1}, \ldots, x_{n}\right] / I\right)=\text { colength of } I=d .
$$


1.2. The Grothendieck Hilbert scheme of points. $\mathbf{H}_{\mathrm{A}_{\mathrm{k}}^{n}}^{d}$, the multigraded Hilbert scheme of points, is a generalization of the "classical" Grothendieck Hilbert scheme Hilb $_{\AA_{\mathrm{k}}^{n}}^{d}$ of $d$ points of $\mathbb{A}_{\mathrm{k}}^{n}$ over a Noetherian ring $\mathrm{k}$ (often an algebraically closed field). The latter scheme can be constructed as an open subscheme of Hilb $_{\mathbb{P}_{k}^{n}}^{d}$ (a projective scheme the existence of which is a consequence of Grothendieck's general construction given in [Grothendieck 1995]; see also, for example, [Eisenbud and Harris 2000, VI.2.2; Kollár 1996, Ch. 1; Miller and Sturmfels 2005, Ch. 18; Sernesi 1986, Ch. 7]) that arises from the inclusion of $\mathbb{A}_{k}^{n}$ in $\mathbb{P}_{k}^{n}$ as a standard affine. We will briefly recall the basics of Hilb $\mathbb{P}_{k}^{d}$, describe the construction of Hilb $_{\mathbb{A}_{k}^{n}}^{d}$ as an open subscheme of Hilb ${\mathbb{\mathbb { P } _ { \mathrm { k } } ^ { n }}}^{d}$, and show that $\operatorname{Hilb}_{\mathbb{A}_{\mathrm{k}}^{n}}^{d}$ represents the functor $H_{\mathrm{A}_{\mathrm{k}}^{n}}^{d}$.

Basics of $\operatorname{Hilb}_{\mathbb{P}_{\mathrm{k}}^{n}}^{d}$. Recall that $\mathrm{Hilb}_{\mathbb{P}_{\mathrm{k}}^{n}}^{d}$ represents the functor that assigns to any $k$ scheme $T$ the set of all closed (necessarily, by an easy adaptation of the proof of Lemma 1) subschemes $Z \subseteq T \times \times_{\mathrm{k}} \mathbb{P}_{\mathrm{k}}^{n}$ that are flat and proper over $T$, with fibers having constant Hilbert polynomial $d$ (in particular, $Z$ is quasi-finite over $T$ ). The universal closed subscheme $Z_{\mathbb{P}_{\mathrm{k}}^{n}}^{d} \subseteq \operatorname{Hilb}_{\mathbb{P}_{\mathrm{k}}^{n}}^{d} \times_{\mathrm{k}} \mathbb{P}_{\mathrm{k}}^{n}$ corresponds to the identity map Hilb $_{\mathbb{P}_{\mathrm{k}}^{n}}^{d} \rightarrow \operatorname{Hilb}_{\mathbb{P}_{\mathrm{k}}^{n}}^{d}$, and (Hilb ${\mathbb{\mathbb { P } _ { \mathrm { k } } ^ { n }}}^{d}, Z_{\mathbb{P}_{\mathrm{k}}^{n}}^{d})$ satisfies the following universal property:

The set of maps $f: T \rightarrow \operatorname{Hilb}_{\mathbb{P}_{k}^{n}}^{d}$ is in natural bijective correspondence with the set of closed subschemes $Z_{f} \subseteq T \times \times_{\mathrm{k}} \mathbb{P}_{\mathrm{k}}^{n}$ that are flat and proper over $T$, with fibers having constant Hilbert polynomial $d$; the bijection $f \mapsto Z_{f}$ is defined by $Z_{f}=T \times_{\operatorname{Hilb}_{\mathbb{P}_{k}^{n}}^{d}} Z_{\mathbb{P}_{\mathrm{k}}^{n}}^{d}$.

Construction of $\operatorname{Hilb}_{\mathbb{A}_{\mathrm{k}}^{n}}^{d}$. Let $p: Z_{\mathbb{P}_{\mathrm{k}}^{n}}^{d} \rightarrow \operatorname{Hilb}_{\mathbb{P}_{\mathrm{k}}^{n}}^{d}$ denote the flat and proper map induced by the first projection, and define

$$
\begin{aligned}
W & =\operatorname{Hilb}_{\mathbb{P}_{\mathrm{k}}^{n}}^{d} \backslash p\left(\operatorname{Hilb}_{\mathbb{P}_{\mathrm{k}}^{n}}^{d} \times_{\mathrm{k}}\left(\mathbb{P}_{\mathrm{k}}^{n} \backslash \mathbb{A}_{\mathrm{k}}^{n}\right) \cap Z_{\mathbb{P}_{\mathrm{k}}^{n}}^{d}\right), \\
Z_{W}^{d} & =p^{-1}(W) \subseteq Z_{\mathbb{P}_{\mathrm{k}}^{n}}^{d} ;
\end{aligned}
$$

since $p$ is proper, it maps the closed set on the right of the first displayed line (an intersection of two closed sets) to a closed set, and therefore $W$ is open in $\operatorname{Hilb}_{\mathbb{P}_{\mathrm{k}}^{n}}^{d}$. It is then clear that

$$
Z_{W}^{d} \subseteq W \times_{\mathrm{k}} \mathbb{A}_{\mathrm{k}}^{n} \subseteq \operatorname{Hilb}_{\mathbb{P}_{\mathrm{k}}^{n}}^{d} \times_{\mathrm{k}} \mathbb{A}_{\mathrm{k}}^{n} \subseteq \operatorname{Hilb}_{\mathbb{P}_{\mathrm{k}}^{n}}^{d} \times_{\mathrm{k}} \mathbb{P}_{\mathrm{k}}^{n} .
$$

Suppose we are given a closed subscheme

$$
Z \subseteq T \times{ }_{\mathrm{k}} \mathbb{A}_{\mathrm{k}}^{n} \subseteq T \times \times_{\mathrm{k}} \mathbb{P}_{\mathrm{k}}^{n}
$$

such that $Z$ is flat and proper over $T$ with fibers of constant Hilbert polynomial $d$. Then the induced map $f: T \rightarrow$ Hilb $_{\mathbb{P}_{\mathrm{k}}^{n}}^{d}$ must factor through the inclusion $W \subseteq$ Hilb $_{\mathbb{P}_{\mathrm{k}}^{n}}^{d}$, since the fiber of $Z_{\mathbb{P}_{\mathrm{k}}^{n}}^{d}$ over any point of $\mathrm{Hilb}_{\mathbb{P}_{\mathrm{k}}^{n}}^{d} \backslash W$ must contain a point of 
$\operatorname{Hilb}_{\mathbb{P}_{\mathrm{k}}^{n}}^{d} \times_{\mathrm{k}}\left(\mathbb{P}_{\mathrm{k}}^{n} \backslash \mathbb{A}_{\mathrm{k}}^{n}\right)$. Conversely, a map

$$
T \rightarrow W \subseteq \operatorname{Hilb}_{\mathbb{P}_{\mathrm{k}}^{n}}^{d}
$$

induces by pullback a closed subscheme

$$
Z \subseteq T \times{ }_{\mathrm{Hil}_{\mathbb{P}_{\mathrm{k}}^{n}}^{d}} Z_{\mathbb{P}_{\mathrm{k}}^{n}}^{d}=T \times_{W} Z_{W}^{d} \subseteq T \times \times_{\mathrm{k}} \mathbb{A}_{\mathrm{k}}^{n}
$$

that is flat and proper over $T$ with fibers of constant Hilbert polynomial $d$. In short, the pair $\left(W, Z_{W}^{d}\right)$ represents the "classical" Hilbert functor for $\mathbb{A}_{\mathrm{k}}^{n}$; we write $W=\operatorname{Hilb}_{\AA_{\mathrm{k}}^{n}}^{d}$ and $Z_{W}^{d}=Z_{\AA_{\mathrm{k}}^{n}}^{d}$.

Hilb $_{\AA_{\mathrm{k}}^{n}}^{d}$ represents the functor $H_{\AA_{k}^{n}}^{d}$. Recall that we are for the moment assuming that the ring $\mathrm{k}$ is Noetherian; therefore $\mathrm{Hilb}_{\mathbb{P}_{\mathrm{k}}^{n}}^{d}$ and $\mathrm{Hilb}_{\AA_{\mathrm{k}}^{n}}^{d}$ are Noetherian. Moreover, the map $Z_{\mathrm{A}_{\mathrm{k}}^{n}}^{d} \rightarrow \mathrm{Hilb}_{\mathrm{A}_{\mathrm{k}}^{n}}^{d}$ is finite, since it is quasi-finite and proper (see, for example, [Eisenbud and Harris 2000, p. 92] or [Fulton 1984, App. B.2.4]); it follows from [Mumford 1966, Prop. 7, p. 43] that the direct image $2_{\mathbb{A}_{k}^{n}}^{d}$ of the structure sheaf of $Z_{\mathrm{A}_{k}^{n}}^{d}$ is locally free; the rank of $2_{\mathrm{A}_{\mathrm{k}}^{n}}^{d}$ is $d$ since the fibers have Hilbert polynomial $d$. Consequently, a map $f: T \stackrel{A_{k}^{n}}{\rightarrow} \operatorname{Hilb}_{\AA_{k}^{n}}^{d}$ corresponds to a closed subscheme

$$
Z_{f}=T \times_{\operatorname{Hill}_{\mathrm{A}_{\mathrm{k}}^{n}}^{d}} Z_{\mathrm{A}_{\mathrm{k}}^{n}}^{d} \subseteq T \times \times_{\mathrm{k}} \mathbb{A}_{\mathrm{k}}^{n},
$$

and the direct image $2_{f}$ of the structure sheaf of $Z_{f}$ under the map $Z_{f} \rightarrow T$ is locally free of rank $d$, since $2_{f}$ is the pullback of the direct image $2_{\mathbb{A}_{k}^{n}}^{d}$.

Conversely, a closed subscheme $Z \subseteq T \times{ }_{k} \mathbb{A}_{\mathrm{k}}^{n}$ such that the direct image of the structure sheaf $\mathrm{O}_{Z}$ on $T$ is locally free of rank $d$, is flat and proper over $T$, with fibers of Hilbert polynomial $d$, and so gives rise to a map $T \rightarrow \operatorname{Hilb}_{A_{k}^{n}}^{d}$.

This demonstrates that Hilb ${ }_{\AA_{\mathrm{k}}^{n}}^{d}$ represents the "multigraded" Hilbert functor $H_{\mathrm{A}_{\mathrm{k}}^{n}}^{d}$ discussed in Section 1.1.

1.3. The main theorem. As previously stated, the goal of this paper is to present an elementary construction of the universal pair $\left(\mathbf{H}_{\mathrm{A}_{\mathrm{k}}^{n}}^{d}, Z_{\mathrm{A}_{\mathrm{k}}^{n}}^{d}\right)$ that uses only polynomial arithmetic. The construction is outlined in the proof of the following theorem.

Theorem 3. Let $\mathrm{k}$ be an arbitrary commutative and unitary ring. Then the functor $H_{A_{k}^{n}}^{d}$ is represented by a scheme $\mathbf{H}_{A_{k}^{n}}^{d}$ that is separated and of finite type over the ring $\mathrm{k}$, together with a closed subscheme $Z_{\mathbb{A}_{k}^{n}}^{d} \subseteq \mathbf{H}_{A_{k}^{n}}^{d} \times{ }_{k} \mathbb{A}_{k}^{n}$ such that the direct image of the structure sheaf of $Z_{\AA_{k}^{n}}^{d}$ on $\mathbf{H}_{A_{k}^{n}}^{d}$ is locally free of rank $d$. That is, the $\operatorname{pair}\left(\mathbf{H}_{\mathbb{A}_{k}^{n}}^{d}, Z_{\AA_{k}^{n}}^{d}\right)$ satisfies the universal property (1).

Proof. We construct $\mathbf{H}_{\mathrm{A}_{\mathrm{k}}^{n}}^{d}$ by gluing together subschemes that represent open subfunctors of the functor $H_{\AA_{k}^{n}}^{d}$. Each subfunctor corresponds to a basis set $\beta$ of $d$ 
monomials in the variables $x_{1}, \ldots, x_{n}$; that is, $\beta$ has the property that whenever $m \in \beta$ and $m_{1} \mid m$, then $m_{1} \in \beta$. Given a basis set $\beta$, we define the subfunctor

$$
F_{\mathrm{A}_{\mathrm{k}}^{n}}^{\beta}: \underline{\mathrm{k}-\mathrm{Sch}} \rightarrow \underline{\mathrm{Set}}
$$

of $H_{\AA_{k}^{n}}^{d}$ by stipulating that $F_{\AA_{k}^{n}}^{\beta}(T) \subseteq H_{\AA_{k}^{n}}^{d}(T)$ is the subset of closed subschemes $Z \subseteq T \times{ }_{k} A_{k}^{n}$ such that the direct image of the structure sheaf of $Z$ on $T$ is free with basis $\beta$ (see Section 2.1). The technical heart of the paper is the demonstration that the subfunctors $F_{\mathbb{A}_{k}^{n}}^{\beta}$ are representable by pairs $\left(U_{\beta}, Z_{\beta}\right)$; the gluing argument is relatively straightforward.

The theorem is thus an immediate consequence of Lemma 7 (which carries out the gluing) and Theorem 37 (which completes the construction of the universal pairs $\left(U_{\beta}, Z_{\beta}\right)$ that extends over several sections).

Lemma 7. Suppose that for all basis sets $\beta \subseteq \mathrm{k}[\mathbf{x}]$ of size $d$, the functor $F_{\mathbb{A}_{\mathrm{k}}^{n}}^{\beta}$ is represented by an affine $\mathrm{k}$-scheme $U_{\beta}=\operatorname{Spec}\left(R_{\beta}\right)$, and universal closed subscheme $Z_{\beta} \subseteq U_{\beta} \times_{\mathrm{k}} \mathbb{A}_{\mathrm{k}}^{n}$. Furthermore, suppose that the schemes $U_{\beta}$ are of finite type over $\mathrm{k}$ (that is, the rings $R_{\beta}$ are finitely generated $\mathrm{k}$-algebras). Then there exists a kscheme $\mathbf{H}_{\AA_{k}^{n}}^{d}$ that is separated and of finite type over $\mathrm{k}$, and a closed subscheme $Z_{\AA_{k}^{n}}^{d} \subseteq \mathbf{H}_{\mathbb{A}_{k}^{n}}^{d} \times_{k} A_{k}^{n}$, that together satisfy the universal property (1).

Theorem 37. Let $\beta \subseteq \mathrm{k}[x]$ be a basis set of $d$ monomials. Then there exists a pair $\left(U_{\beta}, Z_{\beta}\right)$ consisting of an affine scheme $U_{\beta}$ of finite type over $\mathrm{k}$, and a closed subscheme $Z_{\beta} \subseteq U_{\beta} \times_{\mathrm{k}} \mathbb{A}_{\mathrm{k}}^{n}$, that represents the functor $F_{\mathrm{A}_{\mathrm{k}}^{n}}^{\beta}$.

1.4. Sketch of the construction of $\left(U_{\beta}, Z_{\beta}\right)$. We now present a sketch of the construction of the universal pair $\left(U_{\beta}, Z_{\beta}\right)$ in the special case $\beta=\left\{1, x_{1}\right\} \subseteq \mathrm{k}\left[x_{1}, x_{2}\right]$, to give a more detailed sense of the contents of the paper (notation used is that of later sections). Roughly speaking, we want $U_{\beta}=\operatorname{Spec}\left(R_{\beta}\right)=\operatorname{Spec}(R)$ to be the base scheme of the "universal" closed subscheme $Z_{\beta} \subseteq U_{\beta} \times{ }_{\mathrm{k}} \mathbb{A}_{\mathrm{k}}^{n}$ such that the direct image of the structure sheaf of $Z_{\beta}$ on $U_{\beta}$ is free with basis $\beta$. In other words, if

$$
\widehat{J} \subseteq R \otimes_{\mathrm{k}} \mathrm{k}\left[x_{1}, x_{2}\right]=R\left[x_{1}, x_{2}\right]
$$

is the ideal cutting out $Z_{\beta}$, then the quotient $R\left[x_{1}, x_{2}\right] / \widehat{J}$ should be $R$-free with basis $\beta$, and "universal" among such quotients.

Let $B$ be a k-algebra, and consider a quotient $B\left[x_{1}, x_{2}\right] / I$ that is $B$-free with basis $\beta$. Since the quotient is free, we know that every monomial $x_{1}^{r_{1}} x_{2}^{r_{2}}$ is congruent to a unique $B$-linear combination of 1 and $x_{1}$ modulo the ideal $I$. Put another way, $I$ contains a unique polynomial of the form

$$
F_{\left(r_{1}, r_{2}\right)}=x_{1}^{r_{1}} x_{2}^{r_{2}}-c_{(0,0)}^{\left(r_{1}, r_{2}\right)} \cdot 1-c_{(1,0)}^{\left(r_{1}, r_{2}\right)} \cdot x_{1}
$$


for every monomial $x_{1}^{r_{1}} x_{2}^{r_{2}}$. One sees easily (Proposition 11$)$ that $I$ is generated by these polynomials; in fact, $I$ is generated by the three polynomials $F_{(0,1)}, F_{(1,1)}$, and $F_{(2,0)}$ associated to the boundary monomials of $\beta$ (Proposition 12), which are shown in boldface in the following diagram.

$$
\begin{array}{ccc}
\mathbf{x}_{2} & \mathbf{x}_{1} \mathbf{x}_{2} & \\
1 & x_{1} & \mathbf{x}_{1}^{2} .
\end{array}
$$

From this we see that our quotient $B\left[x_{1}, x_{2}\right] / I$ is determined by a choice of six coefficients

$$
c_{\left(j_{1}, j_{2}\right)}^{\left(r_{1}, r_{2}\right)} \in B, \quad\left(r_{1}, r_{2}\right) \in\{(0,1),(1,1),(2,0)\}, \quad\left(j_{1}, j_{2}\right) \in\{(0,0),(1,0)\} .
$$

However, these coefficients cannot be chosen arbitrarily, for the following reason. Consider the following $B\left[x_{1}, x_{2}\right]$-linear combination of our three ideal generators.

$$
\begin{aligned}
& -x_{1} \cdot F_{(0,1)}+1 \cdot F_{(1,1)}-c_{(1,0)}^{(0,1)} \cdot F_{(2,0)} \\
& \quad\left(-c_{(0,0)}^{(1,1)}+c_{(1,0)}^{(0,1)} c_{(0,0)}^{(2,0)}\right) \cdot 1+\left(c_{(0,0)}^{(0,1)}-c_{(1,0)}^{(1,1)}+c_{(1,0)}^{(0,1)} c_{(1,0)}^{(2,0)}\right) \cdot x_{1}
\end{aligned}
$$

Since the result is a $B$-linear combination of the monomials in $\beta$, we call the tuple of coefficients $\left(-x_{1}, 1,-c_{(1,0)}^{(0,1)}\right)$ a pseudosyzygy of the polynomials $F_{(0,1)}, F_{(1,1)}$, and $F_{(2,0)}$. Since the left-hand side is an element of the ideal $I$, so is the righthand side; that is, the right-hand side is congruent to 0 modulo $I$. But the quotient $B\left[x_{1}, x_{2}\right] / I$ is supposed to be $B$-free with basis $\beta$, which means that the only $B$ linear combination of 1 and $x_{1}$ that can be congruent to 0 modulo $I$ is the trivial one. It follows that the right-hand side is equal to 0 , and therefore the coefficients of 1 and $x_{1}$ must be 0 in $B$, which yields the following polynomial relations on the coefficients (3).

$$
\begin{aligned}
-c_{(0,0)}^{(1,1)}+c_{(1,0)}^{(0,1)} c_{(0,0)}^{(2,0)} & =0, \\
c_{(0,0)}^{(0,1)}-c_{(1,0)}^{(1,1)}+c_{(1,0)}^{(0,1)} c_{(1,0)}^{(2,0)} & =0 .
\end{aligned}
$$

Equivalently, the pseudosyzygy must be a syzygy of the polynomials $F_{(0,1)}$, $F_{(1,1)}$, and $F_{(2,0)}$. (This is a special case of Lemma 13.) The reader can check that the following tuple of coefficients is also a pseudosyzygy — and hence a syzygy of the polynomials:

$$
\left(c_{(0,0)}^{(2,0)}, c_{(1,0)}^{(2,0)}-x_{1},-c_{(1,0)}^{(1,1)}+x_{2}\right) .
$$

The polynomial relations associated to this syzygy are

$$
\begin{aligned}
-c_{(0,0)}^{(0,1)} c_{(0,0)}^{(2,0)}+c_{(1,0)}^{(1,1)} c_{(0,0)}^{(2,0)}-c_{(0,0)}^{(1,1)} c_{(1,0)}^{(2,0)} & =0, \\
c_{(0,0)}^{(1,1)}-c_{(1,0)}^{(0,1)} c_{(0,0)}^{(2,0)} & =0 .
\end{aligned}
$$


The two pseudosyzygies presented are the basic pseudosyzygies in this case. (As syzygies, they in fact generate the full $B\left[x_{1}, x_{2}\right]$-module of syzygies of the three generators of the ideal $I$; this is part of the first statement of Theorem 22.)

So how do we construct the universal free quotient? We want the coefficients (3) to be as "generic" as possible. Therefore, we introduce a set of six indeterminates

$$
\mathbf{C}=\left\{C_{\left(j_{1}, j_{2}\right)}^{\left(r_{1}, r_{2}\right)} \mid\left(r_{1}, r_{2}\right) \in\{(0,1),(1,1),(2,0)\},\left(j_{1}, j_{2}\right) \in\{(0,0),(1,0)\}\right\},
$$

and let the ideal cutting out the universal family be generated by the functions

$$
G_{\left(r_{1}, r_{2}\right)}=x_{1}^{r_{1}} x_{2}^{r_{2}}-C_{(0,0)}^{\left(r_{1}, r_{2}\right)} \cdot 1-C_{(1,0)}^{\left(r_{1}, r_{2}\right)} \cdot x_{1}, \quad\left(r_{1}, r_{2}\right) \in\{(0,1),(1,1),(2,0)\} .
$$

However, we know that to get a free quotient, it is necessary that the basic pseudosyzygies be syzygies, which imposes the polynomial relations (4) and (5) on the indeterminates $C_{j_{1}, j_{2}}^{r_{1}, r_{2}}$ (replace the $c$ 's by the corresponding $C$ 's). Let $\Re \subseteq \mathrm{k}[\mathbf{C}]$ denote the ideal generated by these polynomials. The coordinate ring of the scheme $U_{\beta}$ is then

$$
R=R_{\beta}=\mathrm{k}[\mathbf{C}] / \mathfrak{R},
$$

and the ideal cutting out the universal family $Z_{\beta}$ is

$$
\widehat{J}=\left(G_{(0,1)}, G_{(1,1)}, G_{(2,0)}\right) \subseteq R\left[x_{1}, x_{2}\right] .
$$

To see that this works, one still has to check two details:

(1) That the quotient $R\left[x_{1}, x_{2}\right] / \widehat{J}$ is in fact $R$-free with basis $\beta$.

(2) That the pair $\left(U_{\beta}, Z_{\beta}\right)$ represents the functor $F_{\mathrm{A}_{k}^{n}}^{\beta}$.

To check the first detail, one proves that it is sufficient for a free quotient that the basic pseudosyzygies be syzygies; this is the last statement of Theorem 22 . (The basic pseudosyzygies of the $G$ 's are syzygies over $R$ by construction.) The second detail is checked in general in the proof of Theorem 37; the basic idea can be seen by returning to the free quotient $B\left[x_{1}, x_{2}\right] / I$ considered earlier. If we let $T=\operatorname{Spec}(B)$, then the closed subscheme

$$
Z=\operatorname{Spec}\left(B\left[x_{1}, x_{2}\right] / I\right) \subseteq T \times{ }_{\mathrm{k}} \mathbb{A}_{\mathrm{k}}^{n}
$$

is an element of $F_{\mathrm{A}_{\mathrm{k}}^{n}}^{\beta}(T)$, and so must correspond to a unique map $T \rightarrow U_{\beta}$ such that $Z$ is the pullback of $Z_{\beta}$. One checks that the desired map is induced by the map

$$
\mathrm{k}[\mathbf{C}] \rightarrow B, \quad C_{\left(j_{1}, j_{2}\right)}^{\left(r_{1}, r_{2}\right)} \mapsto c_{\left(s_{1}, s_{2}\right)}^{\left(r_{1}, r_{2}\right)},
$$

under which the ideal $\mathfrak{R}$ maps to 0 , because the basic pseudosyzygies of the three polynomials $F_{(0,1)}, F_{(1,1)}$, and $F_{(2,0)}$ (that generate $I$ ) are all syzygies. 
Before leaving this section, we extend our discussion of the example a little further. One checks that the ideal

$$
\mathfrak{R}=\left(-C_{(0,0)}^{(1,1)}+C_{(1,0)}^{(0,1)} C_{(0,0)}^{(2,0)}, C_{(0,0)}^{(0,1)}-C_{(1,0)}^{(1,1)}+C_{(1,0)}^{(0,1)} C_{(1,0)}^{(2,0)}\right)
$$

is generated by just two of the original generators, as shown. From this it follows that

$$
\mathrm{k}[\mathbf{C}] / \mathfrak{R}=\mathrm{k}\left[C_{(1,0)}^{(0,1)}, C_{(1,0)}^{(1,1)}, C_{(0,0)}^{(2,0)}, C_{(1,0)}^{(2,0)}\right]
$$

is a polynomial ring in the four indicated variables; whence, $U_{\beta}$ is isomorphic to $A_{k}^{4}$. See Section 8.3 for another example of the same phenomenon.

1.5. Connection to previous papers. M. Haiman [1998] introduced and studied the schemes $U_{\beta}$ in the case of the affine plane (that is, $n=2$ variables, and $\mathrm{k}$ an algebraically closed field). In [Huibregtse 2002], we gave a fuller description of the $U_{\beta}$ in this case; in particular, we expressed the coordinate rings of the $U_{\beta}$ concretely as quotients of polynomial rings. As an application, we presented conditions on $\beta$ sufficient to imply that $U_{\beta}$ is isomorphic to a $2 d$-dimensional affine space (the example considered in Section 1.4 is of this type). The orientation in [Huibregtse 2002] was descriptive; that is, we began with the (known) existence and basic properties of the Hilbert scheme, and on that basis, we studied the open subschemes $U_{\beta}$.

By contrast, the orientation of the present paper is constructive. We establish the existence of the schemes $U_{\beta}$ in an elementary fashion, independent of the existence of $\mathbf{H}_{\AA_{\mathrm{k}}^{n}}^{d}$; in so doing, we obtain a concrete description of the coordinate rings of the $U_{\beta}$, as before. As previously explained, we construct the pair $\left(U_{\beta}, Z_{\beta}\right)$ to represent the functor $F_{\mathbb{A}_{k}^{n}}^{\beta}$ over an arbitrary commutative, unitary ring k; the entire Hilbert scheme $\mathbf{H}_{\mathrm{A}_{\mathrm{k}}^{n}}^{d}$ is then constructed by patching, yielding a special case of the multigraded Hilbert scheme of Haiman and Sturmfels [2004]. In addition to the shift from description to construction, the present paper also goes beyond [Huibregtse 2002] by treating all cases $n \geq 1$ in a uniform fashion, rather than just the case $n=2$.

Note 4. Shortly after submitting the final version of this article in June 2005, I learned of a preprint [Gustavsen et al. 2005] that presents another elementary proof of the existence of Hilbert schemes of points.

\section{Reduction to the construction of the pairs $\left(U_{\beta}, Z_{\beta}\right)$}

Let $\mathrm{k}$ be a commutative ring and let

$$
S=\mathrm{k}\left[x_{1}, \ldots, x_{n}\right]=\mathrm{k}[\mathbf{x}], \quad \mathbb{A}_{\mathrm{k}}^{n}=\operatorname{Spec}(\mathrm{k}[\mathbf{x}]) .
$$


Recall from Section 1.1 that $\mathbf{H}_{\mathrm{A}_{k}^{n}}^{d}$, the multigraded Hilbert scheme of $d$ points of $\mathbb{A}_{k}^{n}$, together with the universal closed subscheme $Z_{\mathbb{A}_{k}^{n}}^{d} \subseteq \mathbf{H}_{A_{k}^{n}}^{d} \times{ }_{k} \mathbb{A}_{k}^{n}$, represents the functor $H_{\AA_{k}^{n}}^{d}: \underline{\mathrm{k}-\mathrm{Sch}} \rightarrow \underline{\text { Set}}$, defined by

$$
H_{\AA_{\mathrm{k}}^{n}}^{d}(T)=\left\{\begin{array}{l|l}
\text { closed subschemes } Z \subseteq T \times \times_{\mathrm{k}} \mathbb{A}_{\mathrm{k}}^{n} & \begin{array}{l}
\text { the direct image of } \mathrm{O}_{Z} \text { on } T \\
\text { is locally free of rank } d,
\end{array}
\end{array}\right\},
$$

where $\mathrm{O}_{Z}$ denotes the structure sheaf of $Z$. Our goal in this paper is to construct the pair $\left(\mathbf{H}_{\mathrm{A}_{\mathrm{k}}^{n}}^{d}, Z_{\mathrm{A}_{k}^{n}}^{d}\right)$ in an elementary fashion, using essentially only polynomial arithmetic. We take the first step in this section, by reducing the problem of representing the functor $H_{\AA_{k}^{n}}^{d}$ to the problem of representing certain of its sub-functors.

2.1. Subfunctors of $\boldsymbol{H}_{A_{\mathrm{k}}^{n}}^{\boldsymbol{d}}$. Recall that a basis set $\beta$ of monomials is defined by the property that if $m \in \beta$ and a monomial $m_{1} \mid m$, then $m_{1} \in \beta$. For each basis set $\beta$ of $d$ monomials in $x_{1}, \ldots, x_{n}$, we define the functor $F_{\mathrm{A}_{\mathrm{k}}^{n}}^{\beta}$ as follows.

$$
\begin{aligned}
& F_{\mathrm{A}_{\mathrm{k}}^{n}}^{\beta}: \underline{\mathrm{k}-\mathrm{Sch}} \rightarrow \underline{\mathrm{Set}}, \\
& F_{\mathrm{A}_{\mathrm{k}}^{n}}^{\beta}(T)=\left\{\begin{array}{l|l}
\text { closed subschemes } Z \subseteq T \times{ }_{\mathrm{k}} \mathbb{A}_{\mathrm{k}}^{n} & \begin{array}{l}
\text { the direct image of } \mathrm{O}_{Z} \\
\text { on Tis free with basis } \beta .
\end{array}
\end{array}\right\}
\end{aligned}
$$

Assuming that each functor $F_{\AA_{k}^{n}}^{\beta}$ is represented by a pair $\left(U_{\beta}, Z_{\beta}\right)$, such that $U_{\beta}$ is a k-scheme and $Z_{\beta} \subseteq U_{\beta} \times{ }_{k} A_{k}^{n}$ is the universal closed subscheme, we obtain (in Lemma 7) the scheme $\mathbf{H}_{\mathrm{A}_{k}^{n}}^{d}$ by gluing the pairs $\left(U_{\beta}, Z_{\beta}\right)$ together along the isomorphic open subschemes on which they "overlap." In order to identify these subschemes and to verify the compatibility conditions that permit the gluing, we must consider a generalization of the functors (6). Let

$$
\mathfrak{B}=\left\{\beta_{1}, \beta_{2}, \ldots, \beta_{s}\right\}
$$

be a set of $s \geq 1$ basis sets, each of which has cardinality $d$, and define the functor $F_{\mathbb{A}_{k}^{n}}^{\mathfrak{B}}$ as follows.

$$
\begin{aligned}
& F_{A_{k}^{\mathfrak{B}}}^{\mathfrak{k}}: \underline{\mathrm{k}-\mathrm{Sch}} \rightarrow \underline{\text { Set}},
\end{aligned}
$$

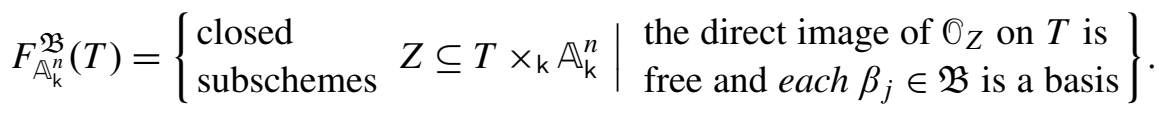

2.2. $\boldsymbol{F}_{\mathrm{A}_{\mathrm{k}}^{n}}^{\boldsymbol{\beta}}$ representable implies $\boldsymbol{F}_{\mathrm{A}_{\mathrm{k}}^{n}}^{\mathfrak{B}}$ representable. Let $\beta \subseteq \mathrm{k}[\mathbf{x}]$ be a basis set consisting of $d$ monomials, and suppose that we have shown that the functor $F_{\mathrm{A}_{\mathrm{k}}^{n}}^{\beta}$ from (6) is represented by an affine k-scheme $U_{\beta}=\operatorname{Spec}(R)$, together with a universal closed subscheme $Z_{\beta} \subseteq U_{\beta} \times_{\mathrm{k}} \mathbb{A}_{\mathrm{k}}^{n}$. Let $2_{\beta}$ denote the direct image on $U_{\beta}$ of the structure sheaf $0_{Z_{\beta}}$, so that $2_{\beta}$ is free with basis $\beta$. Given another basis set $\beta^{\prime} \subseteq \mathrm{k}[\mathbf{x}]$ of size $d$, we can view the monomials in $\beta^{\prime}$ as global sections of 
$2_{\beta}$; therefore, viewing $\beta$ and $\beta^{\prime}$ as column vectors, there is a unique $(d \times d)$-matrix $P=\left(p_{i j}\right)$ of elements of $R$ such that $\beta^{\prime}=P \cdot \beta$. Let $\delta=\operatorname{det}(P)$, and let

$$
W_{\beta^{\prime}}=\operatorname{Spec}\left(R_{\delta}\right) \subseteq U_{\beta}
$$

be the principal open subset on which $\delta$ does not vanish. It is then clear that, as a subset,

$$
W_{\beta^{\prime}}=\left\{\begin{array}{l|l}
x \in U_{\beta} & \begin{array}{l}
\text { the stalk } 2_{\beta}, x \text { is } \mathscr{O}_{U_{\beta}, x} \text {-free } \\
\text { with basis } \beta^{\prime}
\end{array}
\end{array}\right\},
$$

and $W_{\beta^{\prime}}$ is the largest open subscheme on which the restriction of $2_{\beta}$ is free with basis $\beta^{\prime}$. (Note in particular that $W_{\beta}=U_{\beta}$.) Moreover, we have the following lemma.

Lemma 5. Let $T$ be a k-scheme, $f: T \rightarrow U_{\beta}$ a map of $\mathrm{k}$-schemes corresponding to the closed subscheme $Z_{f} \subseteq T \times \times_{k} \mathbb{A}_{k}^{n}$, and $2_{T}$ the direct image of the structure sheaf of $Z_{f}$ on $T$. Then, for any $t \in T$, the stalk $\mathscr{2}_{T, t}$ is $\mathbb{O}_{t}$-free with basis $\beta^{\prime}$ if and only if $f(t) \in W_{\beta^{\prime}}$.

Proof. We denote the residue fields at $t$ and $f(t)$ by $k(t)$ and $k(f(t))$.

$(\Leftarrow)$ : If $f(t) \in W_{\beta^{\prime}}$, then by definition the stalk $2_{\beta, f(t)}$ has the set $\beta^{\prime}$ as $\mathscr{O}_{f(t)^{-}}$ basis. It follows that the fiber of $Z_{\beta}$ over $f(t)$ is $\operatorname{Spec}(A)$, where $A=2_{\beta, f(t)} \otimes_{\mathbb{O}_{f(t)}}$ $k(f(t))$ is an Artin ring that is $k(f(t))$-free with basis $\beta^{\prime}$. Since the fiber of $Z_{f}$ over $t$ is $\operatorname{Spec}\left(A \otimes_{k(f(t))} k(t)\right)$, we have that the latter ring is $k(t)$-free with basis $\beta^{\prime}$. This implies by Nakayama's Lemma that $2_{T, t}$ is $\mathcal{O}_{t}$-free with basis $\beta^{\prime}$.

$(\Rightarrow)$ : Arguing by the contrapositive, suppose that $f(t) \notin W_{\beta^{\prime}}$. Then $\beta^{\prime}$ is not a local basis of $2_{\beta}$ at $f(t)$; therefore, $\beta^{\prime}$ is a $k(f(t))$-linearly dependent set in $A=2_{\beta, f(t)} \otimes_{\mathbb{O}_{f(t)}} k(f(t))$, which has dimension $d$ over $k(f(t))$. Since the map $k(f(t)) \rightarrow k(t)$ is injective, we conclude that $\beta^{\prime}$ is a linearly dependent set in $A \otimes_{k(f(t))} k(t)$, whence $2_{T, t}$ cannot be $\mathscr{O}_{t}$-free with basis $\beta^{\prime}$.

Now let

$$
\mathfrak{B}=\left\{\beta=\beta_{1}, \beta_{2}, \ldots, \beta_{s}\right\}
$$

be a set of $s \geq 1$ basis sets of size $d$, where the functor $F_{\mathrm{A}_{k}^{n}}^{\beta}$ is assumed to be representable as described above. Then the functor $F_{A_{k}^{n}}^{\mathfrak{B}}$ is representable:

Lemma 6. The functor $F_{\mathbb{A}_{k}^{n}}^{\mathfrak{B}}$ is represented by the pair $\left(U_{\mathfrak{B}}, Z_{\mathfrak{B}}\right)$, where

$$
U_{\mathfrak{B}}=\bigcap_{j=1}^{s}\left\{W_{\beta_{j}}\right\} \subseteq U_{\beta},
$$

and $Z_{\mathfrak{B}}$ is the restriction of $Z_{\beta}$ to $U_{\mathfrak{B}}$.

Proof. Suppose given a k-scheme $T$ and a closed subscheme $Z \subseteq T \times{ }_{\mathrm{k}} \mathbb{A}_{\mathrm{k}}^{n}$ such that the direct image $2_{T}$ of $O_{Z}$ on $T$ is free, with each of the bases in $\mathfrak{B}$ as basis. In particular, $2_{T}$ is free with basis $\beta_{1}=\beta$; therefore, the universal property of $\left(U_{\beta}, Z_{\beta}\right)$ yields a unique map $f: T \rightarrow U_{\beta}$ such that $Z=Z_{f}$ is the pullback of 
$Z_{\beta}$ under $f$. Since for every $t \in T$ the stalk $\mathscr{2}_{T, t}$ is $k(t)$-free with basis $\beta_{j}$, for $1 \leq j \leq s$, Lemma 5 implies that the map $f$ factors through $U_{\mathfrak{B}}$; whence, $Z_{f}$ is the pullback of $Z_{\mathfrak{B}}$. Conversely, given a map $f: T \rightarrow U_{\beta}$ with image in $U_{\mathfrak{B}}$, we pull back $Z_{\mathfrak{B}}$ to obtain $Z_{f} \subseteq T \times{ }_{\mathrm{k}} \mathbb{A}_{\mathrm{k}}^{n}$, and the corresponding direct image $\mathscr{2}_{T}$. Lemma 5 now implies that $2_{T}$ is free with each of the basis sets in $\mathfrak{B}$ being a basis. This shows that the pair $\left(U_{\mathfrak{B}}, Z_{\mathfrak{B}}\right)$ represents the functor $F_{\mathbb{A}_{k}^{n}}^{\mathfrak{B}}$.

2.3. $F_{A_{k}^{n}}^{\beta}$ representable implies $\boldsymbol{H}_{\mathrm{A}_{k}^{n}}^{d}$ representable. The following lemma reduces the construction of the multigraded Hilbert scheme of points to the construction of the pairs $\left(U_{\beta}, Z_{\beta}\right)$ that represent the functors $F_{\mathrm{A}_{k}^{n}}^{\beta}$ from (6).

Lemma 7. Suppose that for all basis sets $\beta \subseteq \mathrm{k}[\mathbf{x}]$ of size $d$, the functor $F_{\mathbb{A}_{k}^{n}}^{\beta}$ is represented by an affine $\mathrm{k}$-scheme $U_{\beta}=\operatorname{Spec}\left(R_{\beta}\right)$, and universal closed subscheme $Z_{\beta} \subseteq U_{\beta} \times_{k} A_{k}^{n}$. Furthermore, suppose that the schemes $U_{\beta}$ are of finite type over $\mathrm{k}$ (that is, the rings $R_{\beta}$ are finitely generated $\mathrm{k}$-algebras). Then there exists $a \mathrm{k}-$ scheme $\mathbf{H}_{\AA_{\mathrm{k}}^{n}}^{d}$ that is separated and of finite type over $\mathrm{k}$, and a closed subscheme $Z_{\mathrm{A}_{k}^{n}}^{d} \subseteq \mathbf{H}_{\mathrm{A}_{\mathrm{k}}^{n}}^{d_{\mathrm{A}}} \times_{\mathrm{k}} A_{\mathrm{k}}^{n}$, that together satisfy the universal property (1).

Proof. We proceed to glue the pairs $\left(U_{\beta}, Z_{\beta}\right)$ together to construct $\left(\mathbf{H}_{\mathbb{A}_{k}^{n}}^{d}, Z_{\mathbb{A}_{k}^{n}}^{d}\right)$; we begin by identifying the "overlaps." For each pair $\left(\beta_{i}, \beta_{j}\right)$ of basis sets of size $d$, we let

$$
U_{\left(\beta_{i}, \beta_{j}\right)}=W_{\beta_{j}} \subseteq U_{\beta_{i}},
$$

where $W_{\beta_{j}}$ is the principal open subscheme from (9), and let

$$
Z_{\left(\beta_{i}, \beta_{j}\right)} \subseteq U_{\left(\beta_{i}, \beta_{j}\right)} \times_{\mathrm{k}} \mathbb{A}_{\mathrm{k}}^{n}
$$

denote the restriction of $Z_{\beta_{i}}$ to $U_{\left(\beta_{i}, \beta_{j}\right)}$. By Lemma 6 , the pairs

$$
\left(U_{\left(\beta_{i}, \beta_{j}\right)}, Z_{\left(\beta_{i}, \beta_{j}\right)}\right), \quad\left(U_{\left(\beta_{j}, \beta_{i}\right)}, Z_{\left(\beta_{j}, \beta_{i}\right)}\right)
$$

both represent the functor $F_{\mathrm{A}_{k}^{n}}^{\left\{\beta_{i}, \beta_{j}\right\}}$ in (8). Hence there is a canonical isomorphism

$$
\varphi_{i j}: U_{\left(\beta_{i}, \beta_{j}\right)} \rightarrow U_{\left(\beta_{j}, \beta_{i}\right)}
$$

over which the universal closed subscheme $Z_{\left(\beta_{2}, \beta_{1}\right)}$ pulls back to $Z_{\left(\beta_{1}, \beta_{2}\right)}$; we let $\psi_{i j}: Z_{\left(\beta_{1}, \beta_{2}\right)} \rightarrow Z_{\left(\beta_{2}, \beta_{1}\right)}$ denote the second projection map

$$
\psi_{i j}: Z_{\left(\beta_{1}, \beta_{2}\right)}=U_{\left(\beta_{1}, \beta_{2}\right)} \times_{\left.U_{\left(\beta_{2}, \beta_{1}\right)}\right)} Z_{\left(\beta_{2}, \beta_{1}\right)} \longrightarrow Z_{\left(\beta_{2}, \beta_{1}\right)} ;
$$

it is clear that

$$
\varphi_{i j}^{-1}=\varphi_{j i}, \quad \psi_{i j}^{-1}=\psi_{j i} .
$$

For any triple $\left(\beta_{i}, \beta_{j}, \beta_{k}\right)$ of basis sets of size $d$, note that

$$
U_{\left(\beta_{i}, \beta_{j}, \beta_{k}\right)}=U_{\left(\beta_{i}, \beta_{j}\right)} \cap U_{\left(\beta_{i}, \beta_{k}\right)}=W_{\beta_{i}} \cap W_{\beta_{j}} \cap W_{\beta_{k}}
$$


in the notation of Section 2.2. It follows easily from Lemma 5 that

$$
\varphi_{i j}\left(U_{\left(\beta_{i}, \beta_{j}, \beta_{k}\right)}\right) \subseteq U_{\left(\beta_{j}, \beta_{i}, \beta_{k}\right)} ;
$$

whence, $\varphi_{i j}$ restricts to an isomorphism

$$
\varphi_{i j k}: U_{\left(\beta_{i}, \beta_{j}, \beta_{k}\right)} \rightarrow U_{\left(\beta_{j}, \beta_{i}, \beta_{k}\right)} .
$$

Letting $Z_{\left(\beta_{i}, \beta_{j}, \beta_{k}\right)}$ denote the restriction of $Z_{\beta_{i}}$ to $U_{\left(\beta_{i}, \beta_{j}, \beta_{k}\right)}$, we see that the pullback of $Z_{\left(\beta_{j}, \beta_{i}, \beta_{k}\right)}$ under $\varphi_{i j k}$ is $Z_{\left(\beta_{i}, \beta_{j}, \beta_{k}\right)}$. Lemma 6 implies that for all permutations of $i, j$ and $k$, the pair

$$
\left(U_{\left(\beta_{i}, \beta_{j}, \beta_{k}\right)}, Z_{\left(\beta_{i}, \beta_{j}, \beta_{k}\right)}\right)
$$

represents the functor $F_{\mathrm{A}_{k}^{n}}^{\left\{\beta_{i}, \beta_{j}, \beta_{k}\right\}}$; moreover, we have just seen that the restriction maps $\varphi_{i j k}$ are the canonical isomorphisms, to which are associated the restrictions

$$
\psi_{i j k}: Z_{\left(\beta_{i}, \beta_{j}, \beta_{k}\right)} \rightarrow Z_{\left(\beta_{j}, \beta_{i}, \beta_{k}\right)}
$$

of the isomorphisms $\psi_{i j}$. The compatibility conditions

$$
\varphi_{i k j}=\varphi_{j k i} \circ \varphi_{i j k}, \quad \psi_{i k j}=\psi_{j k i} \circ \psi_{i j k}
$$

are therefore immediate, so we can glue the pairs $\left(U_{\beta}, Z_{\beta}\right)$ along the isomorphisms $\left(\varphi_{i j}, \psi_{i j}\right)$ (see, for example, [Eisenbud and Harris 2000, I.2.4, p. 33] or [Hartshorne 1977, Ex. 2.12, p. 80]). We obtain a scheme $\mathbf{H}_{\mathrm{A}_{k}^{n}}^{d}$ and a subscheme $Z_{\mathrm{A}_{\mathrm{k}}^{n}}^{d} \subseteq \mathbf{H}_{\mathrm{A}_{\mathrm{k}}^{n}}^{d} \times_{\mathrm{k}} \mathbb{A}_{\mathrm{k}}^{n}$ such that the direct image $2_{\mathrm{A}_{\mathrm{k}}^{n}}^{d}$ of the structure sheaf of $Z_{\mathrm{A}_{\mathrm{k}}^{n}}^{d}$ on the scheme $\mathbf{H}_{\mathrm{A}_{\mathrm{k}}^{n}}^{d}$ is locally free of rank $d$ - this holds because the restriction of $2_{\mathbb{A}_{k}^{n}}^{d}$ to the open subscheme $U_{\beta}$ is equal to the direct image $2_{\beta}$ of $Z_{\beta}$, which is (by hypothesis) free with basis $\beta$, a basis set of cardinality $d$. In consequence, we find that $Z_{\AA_{k}^{n}}^{d}$ is in fact a closed subscheme of $\mathbf{H}_{\mathrm{A}_{k}^{n}}^{d} \times{ }_{\mathrm{k}} \mathbb{A}_{\mathrm{k}}^{n}$ by the proof of Lemma 1.

It is evident that the scheme $\mathbf{H}_{\mathrm{A}_{k}^{n}}^{d}$ is of finite type over $\mathrm{k}$, since it is covered by the finitely many open subschemes $U_{\beta}$, each of which is by hypothesis an affine scheme of finite type over k. To show that $\mathbf{H}_{\mathrm{A}_{\mathrm{k}}^{n}}^{d}$ is separated over $\operatorname{Spec}(k)$, we use the criterion given by [Grothendieck and Dieudonné 1971, Prop. 5.3.6, p. 281]: it suffices to show that the intersections $U_{\beta_{i}} \cap U_{\beta_{j}}$ in $\mathbf{H}_{\mathbb{A}_{\mathrm{k}}^{n}}^{d}$ are affine, and that the ring

$$
\Gamma\left(U_{\beta_{i}} \cap U_{\beta_{j}},{ }^{\mathbf{H}_{A_{k}^{n}}}\right)
$$

is generated by the union of the images (under the restriction maps) of the rings

$$
\Gamma_{i}=\Gamma\left(U_{\beta_{i}}, O_{\mathbf{H}_{\mathbb{A}_{k}^{n}}^{d}}\right) \quad \text { and } \quad \Gamma_{j}=\Gamma\left(U_{\beta_{j}}, O_{\mathbf{H}_{\mathbb{k}_{\mathrm{k}}^{n}}}\right) .
$$

However, $U_{\beta_{i}} \cap U_{\beta_{j}}$ is isomorphic to the principal open subscheme in (9),

$$
W_{\beta_{j}}=\operatorname{Spec}\left(\Gamma\left(U_{\beta_{i}}, \mathrm{O}_{\mathbf{H}_{\mathrm{A}_{\mathrm{k}}^{n}}}\right){ }_{\delta}\right) \subseteq U_{\beta_{i}},
$$


where $\delta$ is the determinant of the matrix $P$, with entries in $\Gamma_{i}$, such that $\beta_{j}=P \cdot \beta_{i}$ in the sheaf $2_{\beta_{i}}$. The coordinate ring of $W_{\beta_{j}}$ is generated by quotients of the form $a / \delta$, where $a \in \Gamma_{i}$. Since the sheaf $2_{\beta_{j}}$ is free with basis $\beta_{j}$ on $U_{\beta_{j}}$, there is a unique matrix $P^{\prime}$ with entries in $\Gamma_{j}$ such that $\beta_{i}=P^{\prime} \cdot \beta_{j}$. It is then clear that $\delta^{\prime}=\operatorname{det}\left(P^{\prime}\right)$ restricts to $1 / \delta$ on $W_{\beta_{j}}$; whence, the coordinate ring of the intersection is generated by the images of the coordinate rings of $U_{\beta_{i}}$ and $U_{\beta_{j}}$, as desired.

It remains to show that the pair $\left(\mathbf{H}_{\AA_{k}^{n}}^{d}, Z_{\AA_{k}^{n}}^{d}\right)$ satisfies the universal property (1). To this end, let $T$ be a k-scheme, and $Z \subseteq T \times{ }_{k} A_{k}^{n}$ a closed subscheme such that the direct image $2_{T}$ of the structure sheaf of $Z$ is locally free of rank $d$ on $T$. For each basis set $\beta_{i} \subseteq \mathrm{k}[\mathbf{x}]$ of size $d$, let $V_{\beta_{i}} \subseteq T$ denote the open subscheme such that

$t \in V_{\beta_{i}}$ if and only if the stalk $\mathscr{2}_{T, t}$ is $\mathscr{O}_{T, t}$-free with basis $\beta_{i}$.

The $V_{\beta_{i}}$ cover $T$ : indeed, letting $k(t)$ denote the residue field of $t$, the fiber of $Z$ over $\operatorname{Spec}(k(t))$ has the form $\operatorname{Spec}\left(k(t)[\mathbf{x}] / I_{t}\right)$, where the quotient $k(t)[\mathbf{x}] / I_{t}$ is a $k(t)$ vector space of dimension $d$, and accordingly has at least one of the basis sets $\beta_{i}$ as basis (see, for example, [Haiman 1998, Proof of Prop. 2.1, p. 207]). Nakayama's Lemma then implies that $\beta_{i}$ generates $\mathscr{2}_{T, t}$ over $\mathcal{O}_{T, t}$; whence, $t \in V_{\beta_{i}}$.

Let $Z_{V_{\beta_{i}}}$ denote the restriction of $Z$ to $V_{\beta_{i}}$; it is then clear that the direct image of the structure sheaf of $Z_{V_{\beta_{i}}}$ on $V_{\beta_{i}}$ is free with basis $\beta_{i}$. The universal property of $\left(U_{\beta_{i}}, Z_{\beta_{i}}\right)$ then yields a unique map $f_{i}: V_{\beta_{i}} \rightarrow U_{\beta_{i}}$ such that the pullback of $Z_{\beta_{i}}$ is $Z_{V_{\beta_{i}}}$. It is clear that these maps patch to yield a unique map $f_{Z}: T \rightarrow \mathbf{H}_{\AA_{k}^{n}}^{d}$ such that the pullback of $Z_{\AA_{k}^{n}}^{d}$ is $Z$. Conversely, every map $f: T \rightarrow \mathbf{H}_{A_{k}^{n}}^{d}$ induces, by pulling back $Z_{\AA_{\mathrm{k}}^{n}}^{d}$, a closed subscheme $Z_{f} \subseteq T \times{ }_{\mathrm{k}} \mathbb{A}_{\mathrm{k}}^{n}$ such that the direct image of the structure sheaf of $Z_{f}$ on $T$ is locally free of rank $d$. This completes the proof of the lemma.

2.4. Locus of reduced subschemes in $\boldsymbol{U}_{\boldsymbol{\beta}}$. In this subsection we restrict $k$ to be an algebraically closed field, and assume that the construction of the Hilbert scheme $\left(\mathbf{H}_{\AA_{k}^{n}}^{d}, Z_{\AA_{k}^{n}}^{d}\right)$ has been completed. We study the locus of k-points in $U_{\beta} \subseteq \mathbf{H}_{\AA_{k}^{n}}^{d}$ representing reduced closed subschemes of $\mathbb{A}_{k}^{n}$ : subschemes supported at $d$ distinct points $p_{1}, \ldots, p_{d} \in \mathbb{A}_{\mathrm{k}}^{n}$. We write $\left[p_{1}, \ldots, p_{d}\right] \in \mathbf{H}_{\mathbb{A}_{\mathrm{k}}^{n}}^{d}$ for the point corresponding to the closed subscheme determined by the $d$ points $p_{i}$; of course, the subscheme does not depend on the order in which the points are listed. We have the following

Proposition 8. There is a nonempty principal open subscheme

$$
V_{\beta}^{\circ} \subseteq\left(\mathbb{A}_{\mathrm{k}}^{n}\right)^{d}
$$

such that $\left[p_{1}, \ldots, p_{d}\right] \in U_{\beta}$ if and only if $\left(p_{1}, \ldots, p_{d}\right) \in V_{\beta}^{\circ}$.

Proof. Given distinct points $p_{1}, \ldots, p_{d} \in \mathbb{A}_{\mathrm{k}}^{n}$, let

$$
\mathfrak{m}_{i} \subseteq \mathrm{k}\left[x_{1}, \ldots, x_{n}\right]=\mathrm{k}[\mathbf{x}]
$$


denote the maximal ideal of $p_{i}, 1 \leq i \leq d$, and

$$
I=\bigcap_{i=1}^{d} \mathfrak{m}_{i}
$$

be the ideal defining the subscheme $\left[p_{1}, \ldots, p_{d}\right]$. For any $g \in \mathrm{k}[\mathbf{x}]$, we write $g\left(p_{i}\right) \in \mathrm{k}$ for $g$ evaluated at $p_{i}$. Then, by the Chinese Remainder Theorem, the (k-linear) map

$$
\mathrm{k}[\mathbf{x}] / I \rightarrow \bigoplus_{i=1}^{d} \mathrm{k}[\mathbf{x}] / \mathfrak{m}_{i}, \quad g \mapsto\left(g\left(p_{1}\right), \ldots, g\left(p_{d}\right)\right),
$$

is an isomorphism. The basis set $\beta=\left\{m_{1}, \ldots, m_{d}\right\}$ is a $\mathrm{k}$-basis of the quotient $k[\mathbf{x}] / I$ if and only if the determinant of the matrix

$$
\left[\begin{array}{cccc}
m_{1}\left(p_{1}\right) & m_{2}\left(p_{1}\right) & \ldots & m_{d}\left(p_{1}\right) \\
m_{1}\left(p_{2}\right) & m_{2}\left(p_{2}\right) & \ldots & m_{d}\left(p_{2}\right) \\
\vdots & \vdots & & \vdots \\
m_{1}\left(p_{d}\right) & m_{2}\left(p_{d}\right) & \ldots & m_{d}\left(p_{d}\right)
\end{array}\right]
$$

is nonzero. We now replace the coordinates of each point

$$
p_{i}=\left(p_{i 1}, p_{i 2}, \ldots, p_{i n}\right)
$$

with indeterminates $P_{i 1}, \ldots P_{i n}$, and consider the determinant as a polynomial in the $P_{i j}$. This polynomial is nonzero, since it consists of a sum of $d$ ! terms of the form

$$
\pm 1 \cdot \prod_{i=1}^{d} m_{i}\left(P_{\pi(i) 1}, \ldots, P_{\pi(i) n}\right),
$$

where $\pi$ is a permutation of $\{1, \ldots, d\}$, and a moment's reflection shows that the $d$ ! monomials (in the $P_{i j}$ ) appearing in these terms are all distinct. Letting $V_{\beta}^{\circ}$ be the principal open subscheme whose complement is the zero-locus of the determinant, we see that the proposition holds provided that $V_{\beta}^{\circ}$ is nonempty. However, since $\mathrm{k}$ is infinite (being algebraically closed), and the determinant is nonzero, we can find values $p_{i j} \in \mathrm{k}$ that when substituted into the indeterminates $P_{i j}$ make the determinant nonzero (see, for example, [Eisenbud 1995, Sec. 1.6, p. 31]); this implies that $V_{\beta}^{\circ}$ is nonempty, and we are done.

It is clear that the open subscheme $V_{\beta}^{\circ}$ is invariant under the natural action of the symmetric group $S_{d}$ on $\left(\mathbb{A}_{\mathrm{k}}^{n}\right)^{d}$. The quotient map

$$
\pi_{\beta}: V_{\beta}^{\circ} \rightarrow V_{\beta}^{\circ} / S_{d}=W_{\beta}^{\circ}
$$


is finite-to-one, and the k-points $\pi_{\beta}\left(p_{1}, \ldots, p_{d}\right) \in W_{\beta}^{\circ}$ are in bijective correspondence with the points $\left[p_{1}, \ldots, p_{d}\right] \in U_{\beta}$, where the $p_{i}=\left(p_{i 1}, p_{i 2}, \ldots, p_{i n}\right)$ are distinct k-points of $\mathbb{A}_{\mathrm{k}}^{n}$. Therefore, each open subscheme $U_{\beta} \subseteq \mathbf{H}_{\mathrm{A}_{\mathrm{k}}^{n}}^{d}$ meets the principal component $\mathbf{H}_{\circ}^{d} \subseteq \mathbf{H}_{\AA_{k}^{n}}^{d}$ (the closure of the open set of points representing closed subschemes supported at $d$ distinct points of $\mathbb{A}_{k}^{n}$ - see, for example, [Iarrobino 1972, p. 72]) in at least an $(n \cdot d)$-dimensional locus; in particular,

$$
\mathbf{H}_{\circ}^{d} \cap U_{\beta} \neq \varnothing .
$$

We have the following corollary.

Corollary 9. Let $\mathrm{k}$ be an algebraically closed field, and $\beta, \beta^{\prime}$ two basis sets of $d$ monomials in $\mathrm{k}\left[x_{1}, \ldots, x_{n}\right]$. Then

(1) $U_{\beta} \cap \mathbf{H}_{\circ}^{d}$ is open dense in $\mathbf{H}_{\circ}^{d}$;

(2) $\operatorname{dim}\left(U_{\beta}\right) \geq n \cdot d$; and

(3) $U_{\beta} \cap U_{\beta^{\prime}} \neq \varnothing$.

Proof. It is well-known that the irreducible component $\mathbf{H}_{\circ}^{d}$ has dimension $n \cdot d$. Therefore, the non-empty open subset $\mathbf{H}_{\circ}^{d} \cap U_{\beta}$ is dense in $\mathbf{H}_{\circ}^{d}$, as statement (1) asserts, and has dimension $n \cdot d$. Statement (2) is then immediate, and statement (3) follows since the dense open subsets

$$
\mathbf{H}_{\circ}^{d} \cap U_{\beta} \quad \text { and } \quad \mathbf{H}_{\circ}^{d} \cap U_{\beta^{\prime}}
$$

of $\mathbf{H}_{\circ}^{d}$ must intersect nontrivally.

Remark 10. The discussion in Haiman's article [1998, proof of Prop. 2.6], when generalized from 2 to $n$ variables, shows how to find the values of the coordinate functions $C_{\mathbf{j}}^{\mathbf{b}}$ at the point $\left[p_{1}, \ldots, p_{d}\right] \in U_{\beta}$, given a $d$-tuple of points $\left(p_{1}, \ldots, p_{d}\right) \in V_{\beta}^{\circ}$.

\section{Free quotients of polynomial rings with basis $\beta$}

Recall that $\mathrm{k}$ denotes our commutative and unitary ground ring. Let $\beta \subseteq \mathrm{k}[\mathbf{x}]$ be a basis set of size $d$, and let $B$ be a commutative k-algebra. If the pair $\left(U_{\beta}, Z_{\beta}\right)$ exists and represents the functor $F_{A_{k}^{n}}^{\beta}$ from (6), as assumed in Section 2.2 and Section 2.3, then a map $f: \operatorname{Spec}(B) \rightarrow U_{\beta}$ corresponds to a closed subscheme $Z_{f} \subseteq \operatorname{Spec}(B) \times{ }_{\mathrm{k}} \mathbb{A}_{\mathrm{k}}^{n}$ such that the direct image of the structure sheaf of $Z_{f}$ on $\operatorname{Spec}(B)$ is free with basis $\beta$, or, equivalently, to an ideal $I \subseteq B[\mathbf{x}]$ such that the quotient $B[\mathbf{x}] / I$ is $B$-free with basis $\beta$. In this section, we prepare to construct the pairs $\left(U_{\beta}, Z_{\beta}\right)$ by introducing the boundary monomials of $\beta$ and the associated polynomial generators of the ideal $I$, as exemplified in Section 1.4. 
3.1. Notation for monomials. We have already introduced the shorthand $\mathbf{x}$ to represent the list of indeterminates $x_{1}, x_{2}, \ldots, x_{n}$. We will similarly abbreviate the monomial $x_{1}^{r_{1}} x_{2}^{r_{2}} \ldots x_{n}^{r_{n}}$ by $\mathbf{x}^{\mathbf{r}}$, where $\mathbf{r}$ denotes the tuple of exponents $\left(r_{1}, \ldots, r_{n}\right)$, and will relax the notation further by writing, for example, $\mathbf{j} \in \beta$ instead of $\mathbf{x}^{\mathbf{j}} \in \beta$. We will reserve the letter $\mathbf{j}$ for monomials in $\beta$, the letter $\mathbf{b}$ for monomials $\mathbf{x}^{\mathbf{b}}$ in the boundary of $\beta$ (see the next section), and the letter $\mathbf{r}$ for general monomials $\mathbf{x}^{\mathbf{r}}$.

3.2. Boundary monomials of $\boldsymbol{\beta}$. Let $B$ be a commutative k-algebra, and let $I \subseteq$ $B[\mathbf{x}]$ be an ideal such that the quotient $B[\mathbf{x}] / I$ is $B$-free with basis $\beta$. Then every monomial $x_{1}^{r_{1}} x_{2}^{r_{2}} \ldots x_{n}^{r_{n}}=\mathbf{x}^{\mathbf{r}}$ is congruent modulo $I$ to a unique $B$-linear combination

$$
\mathbf{x}^{\mathbf{r}} \equiv \sum_{\mathbf{j} \in \beta} c_{\mathbf{j}}^{\mathbf{r}} \cdot \mathbf{x}^{\mathbf{j}} \quad(\bmod I)
$$

of monomials in $\beta$; equivalently, $I$ contains, for every monomial $\mathbf{x}^{\mathbf{r}}$, a unique polynomial of the form

$$
F_{\mathbf{r}}=\mathbf{x}^{\mathbf{r}}-\sum_{\mathbf{j} \in \beta} c_{\mathbf{j}}^{\mathbf{r}} \cdot \mathbf{x}^{\mathbf{j}}
$$

Proposition 11. The polynomials $F_{\mathbf{r}}$ generate the ideal $I \in B[\mathbf{x}]$.

Proof. Let $J \subseteq I$ be the ideal generated by the polynomials $F_{\mathbf{r}}$. It is then evident that $B[\mathbf{x}] / J$ is generated as a $B$-module by the monomials $\mathbf{x}^{\mathbf{j}}$ in $\beta$; furthermore, the natural surjection $B[\mathbf{x}] \rightarrow B[\mathbf{x}] / I$ induces a surjection $\sigma: B[\mathbf{x}] / J \rightarrow B[\mathbf{x}] / I$ given by $(g+J) \mapsto(g+I)$ for any $g \in B[\mathbf{x}]$. On the other hand, since $B[\mathbf{x}] / I$ is $B$-free with basis $\beta$, we can define a map $\tau: B[\mathbf{x}] / I \rightarrow B[\mathbf{x}] / J$ of $B$-modules by sending $\left(\mathbf{x}^{\mathbf{j}}+I\right)$ to $\left(\mathbf{x}^{\mathbf{j}}+J\right)$ for each $\mathbf{j} \in \beta$ and extending linearly. Since $\tau \circ \sigma$ maps $\left(\mathbf{x}^{\mathbf{j}}+J\right)$ to itself for each $\mathbf{j} \in \beta$, and these elements generate the source as a $B$-module, it follows that $\tau \circ \sigma$ is the identity; whence, the surjection $\sigma$ is also injective, and hence an isomorphism. It follows that $J=I$, as desired.

In fact, the ideal $I$ is generated by a finite subset of the polynomials $F_{\mathbf{r}}$; we now present one convenient such subset. We say that the monomial $\mathbf{x}^{\mathbf{r}}$ is a boundary monomial of $\beta$ provided that $\mathbf{x}^{\mathbf{r}} \notin \beta$ but $\mathbf{x}^{\mathbf{r}} / x_{j} \in \beta$ for at least one variable $x_{j}$. For example, consider the basis set $\beta=\left\{x_{2}^{2}, x_{2}, 1, x_{1}\right\} \subseteq \mathrm{k}\left[x_{1}, x_{2}\right]$. Then the boundary monomials of $\beta$ are the monomials shown in boldface in the diagram

$$
\begin{array}{ccc}
\mathbf{x}_{2}^{\mathbf{3}} & & \\
x_{2}^{2} & \mathbf{x}_{1} \mathbf{x}_{2}^{2} & \\
x_{2} & \mathbf{x}_{1} \mathbf{x}_{2} & \\
1 & x_{1} & \mathbf{x}_{1}^{2}
\end{array}
$$

We write $\mathfrak{B}(\beta)$ for the set of boundary monomials associated to $\beta$; note that it can be generated by the following simple algorithm: Multiply each monomial in 
$\beta$ by each of the variables, and record the products that fall outside of $\beta$, deleting duplicates.

Proposition 12. The set of polynomials $F_{\mathbf{b}}$ such that $\mathbf{b} \in \mathfrak{B}(\beta)$ is a generating set for the ideal $I \subseteq B[\mathbf{x}]$.

Proof. Let $J^{\prime}$ be the ideal generated by the $F_{\mathbf{b}}$. In light of Proposition 11, it suffices to prove that $F_{\mathbf{r}} \in J^{\prime}$ for all monomials $\mathbf{x}^{\mathbf{r}}$. Clearly this is so for the polynomials $F_{\mathbf{b}}$, and also for the polynomials $F_{\mathbf{j}}$ associated to monomials $\mathbf{j} \in \beta$ (by definition $F_{\mathbf{j}}$ is the zero polynomial, since $\mathbf{x}^{\mathbf{j}}$ is congruent to itself modulo $I$ ). Proceeding by induction on the total degree, we suppose that $\mathbf{x}^{\mathbf{r}}$ is a monomial of minimal total degree (greater than zero) such that $F_{\mathbf{r}} \notin J^{\prime}$. Let $x_{i}$ be a variable that appears with a positive exponent in $\mathbf{x}^{\mathbf{r}}$, and let $\mathbf{x}^{\mathbf{r}^{\prime}}=\mathbf{x}^{\mathbf{r}} / x_{i}$. Then by the induction hypothesis we have that

$$
F_{\mathbf{r}^{\prime}}=\mathbf{x}^{\mathbf{r}^{\prime}}-\sum_{\mathbf{j} \in \beta} c_{\mathbf{j}}^{\mathbf{r}^{\prime}} \cdot \mathbf{x}^{\mathbf{j}}=\sum_{\mathbf{b} \in \mathfrak{B}(\beta)} g_{\mathbf{b}}^{\mathbf{r}^{\prime}} \cdot F_{\mathbf{b}}, \quad g_{\mathbf{b}}^{\mathbf{r}^{\prime}} \in B[\mathbf{x}] .
$$

Multiplying the second of the preceding equalities by $x_{i}$, we find that

$$
\mathbf{x}^{\mathbf{r}}-\sum_{\mathbf{j} \in \beta} c_{\mathbf{j}}^{\mathbf{r}^{\prime}} \cdot\left(\mathbf{x}^{\mathbf{j}} \cdot x_{i}\right)=\sum_{\mathbf{b} \in \mathfrak{B}(\beta)}\left(g_{\mathbf{b}}^{\mathbf{r}^{\prime}} \cdot x_{i}\right) \cdot F_{\mathbf{b}}
$$

Now the monomials $\left(\mathbf{x}^{\mathbf{j}} \cdot x_{i}\right)$ are either in $\beta$ or in $\mathfrak{B}(\beta)$ (equal, say, to $\mathbf{x}^{\mathbf{b}}$ ); if, whenever in the latter case, we add the corresponding $c_{\mathbf{j}}^{\mathbf{r}^{\prime}} \cdot F_{\mathbf{b}}$ to both sides, we eventually achieve an equation of the form

$$
\mathbf{x}^{\mathbf{r}}-\sum_{\mathbf{j} \in \beta} \tilde{c}_{\mathbf{j}} \cdot \mathbf{x}^{\mathbf{j}}=\sum_{\mathbf{b} \in \mathfrak{B}(\beta)} \tilde{g}_{\mathbf{b}} \cdot F_{\mathbf{b}} \in J^{\prime} \subseteq I, \quad \tilde{c}_{\mathbf{j}} \in B, \quad \tilde{g}_{\mathbf{b}} \in B[\mathbf{x}] .
$$

Since the left-hand side has the same form as $F_{\mathbf{r}}$ and lies in $I$, it must be equal to $F_{\mathbf{r}}$, since $I$ contains a unique polynomial of this form for every monomial $\mathbf{x}^{\mathbf{r}}$. Therefore $F_{\mathbf{r}} \in J^{\prime}$, which is a contradiction; whence the proposition.

Let $q$ denote the number of elements in $\mathfrak{B}(\beta)$. Then, by the last proposition, the ideal $I$ corresponds to a $B$-point in affine $(q \cdot d)$-space over $B$, namely, the point whose coordinates are the coefficients $c_{\mathbf{j}}^{\mathbf{b}}$ of the polynomials $F_{\mathbf{b}}$. The locus of $B$-points corresponding in this way to ideals $I$ such that $B[\mathbf{x}] / I$ is $B$-free with basis $\beta$ is closed. Central to this paper is the problem of finding generators of an ideal cutting out this locus.

\section{Syzygies of the polynomials $\boldsymbol{F}_{\mathrm{b}}$}

As in Section 3.2, we let $B$ be a commutative k-algebra, and $I \subseteq B[\mathbf{x}]$ an ideal such that the quotient $B[\mathbf{x}] / I$ is $B$-free with basis $\beta$. By Proposition 12, we know 
that the finite set of polynomials (recall (11))

$$
F_{\mathbf{b}}=\mathbf{x}^{\mathbf{b}}-\sum_{\mathbf{j} \in \beta} c_{\mathbf{j}}^{\mathbf{b}} \cdot \mathbf{x}^{\mathbf{j}}, \quad \mathbf{b} \in \mathfrak{B}(\beta),
$$

generates the ideal $I$. In this section, we study the relations among the $F_{\mathbf{b}}$; more precisely, we describe a set of generators of the first syzygy module of the $F_{\mathbf{b}}$. The ideas of this section (and the next) are generalized from [Huibregtse 2002, Sec. 4], which considered the case of two variables.

4.1. Lemma for finding syzygies. Recall that a syzygy of the polynomials $F_{\mathbf{b}}$ is a $q$-tuple (recall that $q$ denotes the number of boundary monomials) of coefficients $\left(g_{\mathbf{b}}\right)_{\mathbf{b} \in \mathfrak{B}(\beta)}$ such that

$$
\left(\sum_{\mathbf{b} \in \mathfrak{B}(\beta)} g_{\mathbf{b}} \cdot F_{\mathbf{b}}\right)=0, \quad g_{\mathbf{b}} \in B[\mathbf{x}] .
$$

The set of all such syzygies is a $B[\mathbf{x}]$-submodule of $B[\mathbf{x}]^{q}$, the first sygyzy module of the $F_{\mathbf{b}}$.

The key result for finding syzygies is the following lemma.

Lemma 13. If the $B[\mathbf{x}]$-linear combination

$$
L=\sum_{\mathbf{b} \in \mathfrak{B}(\beta)} h_{\mathbf{b}} \cdot F_{\mathbf{b}}
$$

of the $F_{\mathbf{b}}$ is equal to a B-linear combination of the monomials in $\beta$, then in fact $L$ is equal to 0 , and the tuple $\left(h_{\mathbf{b}}\right)$ is a syzygy of the polynomials $F_{\mathbf{b}}$.

Proof. The polynomial $L$ lies in the ideal $I$ generated by the $F_{\mathbf{b}}$, and so $L \equiv 0$ modulo $I$. But by hypothesis $B[\mathbf{x}] / I$ is $B$-free with basis $\beta$, so $L$ must be the trivial $B$-linear combination of the monomials in $\beta$, which implies that $L=0$.

4.2. Construction of syzygies. In view of the lemma, we seek to find $B[\mathbf{x}]$-linear combinations of the $F_{\mathbf{b}}$ that involve only monomials in $\beta$ (that is, basis monomials). For example, let $\beta=\left\{x_{2}^{2}, x_{2}, 1, x_{1}\right\} \subseteq \mathrm{k}\left[x_{1}, x_{2}\right]$, and recall that $\mathfrak{B}(\beta)=$ $\left\{x_{2}^{3}, x_{1} x_{2}^{2}, x_{1} x_{2}, x_{1}^{2}\right\}$ by (12). If $I \subseteq B\left[x_{1}, x_{2}\right]$ is such that $B\left[x_{1}, x_{2}\right] / I$ is $B$-free with basis $\beta$, then by Proposition 12 it is generated by four polynomials of the form

$$
\begin{aligned}
& F_{(0,3)}=x_{2}^{3}-c_{(0,2)}^{(0,3)} \cdot x_{2}^{2}-c_{(0,1)}^{(0,3)} \cdot x_{2}-c_{(0,0)}^{(0,3)} \cdot 1-c_{(1,0)}^{(0,3)} \cdot x_{1}, \\
& F_{(1,2)}=x_{1} x_{2}^{2}-c_{(0,2)}^{(1,2)} \cdot x_{2}^{2}-c_{(0,1)}^{(1,2)} \cdot x_{2}-c_{(0,0)}^{(1,2)} \cdot 1-c_{(1,0)}^{(1,2)} \cdot x_{1}, \\
& F_{(1,1)}=x_{1} x_{2}-c_{(0,2)}^{(1,1)} \cdot x_{2}^{2}-c_{(0,1)}^{(1,1)} \cdot x_{2}-c_{(0,0)}^{(1,1)} \cdot 1-c_{(1,0)}^{(1,1)} \cdot x_{1}, \\
& F_{(2,0)}=x_{1}^{2}-c_{(0,2)}^{(2,0)} \cdot x_{2}^{2}-c_{(0,1)}^{(2,0)} \cdot x_{2}-c_{(0,0)}^{(2,0)} \cdot 1-c_{(1,0)}^{(2,0)} \cdot x_{1} .
\end{aligned}
$$


Consider the product

$$
-x_{1} \cdot F_{(0,3)}=-x_{1} x_{2}^{3}+c_{(0,2)}^{(0,3)} \cdot x_{1} x_{2}^{2}+c_{(0,1)}^{(0,3)} \cdot x_{1} x_{2}+c_{(0,0)}^{(0,3)} \cdot x_{1}+c_{(1,0)}^{(0,3)} \cdot x_{1}^{2} .
$$

With reference to the diagram (12), each monomial in $F_{(0,3)}$ has been shifted one place to the right by this operation. We will call the terms that were shifted from "inside $\beta$ " to "outside $\beta$ " exposed terms; in this case they are

$$
c_{(0,2)}^{(0,3)} \cdot x_{1} x_{2}^{2}, \quad c_{(0,1)}^{(0,3)} \cdot x_{1} x_{2}, \quad c_{(1,0)}^{(0,3)} \cdot x_{1}^{2} .
$$

We can remove the exposed terms by adding the appropriate scalar multiples of the $F$ s corresponding to the exposed terms' boundary monomials; more precisely, in the following linear combination, the only term that is not a scalar times a monomial in $\beta$ is $-x_{1} x_{2}^{3}$ :

$$
-x_{1} \cdot F_{(0,3)}-c_{(0,2)}^{(0,3)} \cdot F_{(1,2)}-c_{(0,1)}^{(0,3)} \cdot F_{(1,1)}-c_{(1,0)}^{(0,3)} \cdot F_{(2,0)} .
$$

To remove the term $-x_{1} x_{2}^{3}$, we add $x_{2} \cdot F_{(1,2)}$, which of course "exposes" the terms

$$
-c_{(0,2)}^{(1,2)} x_{2}^{3}, \quad-c_{(1,0)}^{(1,2)} x_{1} x_{2}
$$

by vertical shifting. We remove these exposed terms as before, and conclude that the expression

$$
\left(-x_{1}+c_{(0,2)}^{(1,2)}\right) \cdot F_{(0,3)}+\left(x_{2}-c_{(0,2)}^{(0,3)}\right) \cdot F_{(1,2)}+\left(c_{(1,0)}^{(1,2)}-c_{(0,1)}^{(0,3)}\right) \cdot F_{(1,1)}-c_{(1,0)}^{(0,3)} \cdot F_{(2,0)}
$$

is equal to a $B$-linear combination of monomials in $\beta$. Therefore, the coefficients in this expression are a syzygy of the $F_{\mathbf{b}}$, by Lemma 13 . Similarly, we can remove the exposed terms from the expressions

$$
\left(x_{2} \cdot F_{(1,1)}-1 \cdot F_{(1,2)}\right) \quad \text { and } \quad\left(-x_{1} \cdot F_{(1,1)}+x_{2} \cdot F_{(2,0)}\right)
$$

to obtain syzygies from the coefficients of the expressions

$$
c_{(0,2)}^{(1,1)} \cdot F_{(0,3)}-1 \cdot F_{(1,2)}+\left(x_{2}+c_{(1,0)}^{(1,1)}\right) \cdot F_{(1,1)}+0 \cdot F_{(2,0)}
$$

and

$c_{(0,2)}^{(2,0)} \cdot F_{(0,3)}-c_{(0,2)}^{(1,1)} \cdot F_{(1,2)}+\left(-x_{1}-c_{(0,1)}^{(1,1)}+c_{(1,0)}^{(2,0)}\right) \cdot F_{(1,1)}+\left(x_{2}-c_{(1,0)}^{(1,1)}\right) \cdot F_{(2,0)}$,

respectively, each of which is equal to a $B$-linear combination of basis monomials.

We claim that the three syzygies found so far (the basic syzygies in this case) generate the full $B\left[x_{1}, x_{2}\right]$-module of syzygies of the polynomials $F_{(0,3)}, F_{(1,2)}, F_{(1,1)}$, and $F_{(2,0)}$. To prove this, and to lay the groundwork for our construction of the pair $\left(U_{\beta}, Z_{\beta}\right)$, we must undertake a more general treatment of the syzygy-making process, beginning with the next section. (Note that in the case of two variables, 
the basic syzygies are in fact free generators of the syzygy module; this is proved in [Huibregtse 2002, Sec. 4]. This is not so in three or more variables.)

\section{Pseudosyzygies}

5.1. Definition of pseudosyzygy. Again we let $B$ be a commutative k-algebra, and $\beta$ a basis set of monomials (in the variables $x_{1}, \ldots, x_{n}$ ) of cardinality $d$. Suppose that one is given arbitrary polynomials

$$
G_{\mathbf{b}}=\mathbf{x}^{\mathbf{b}}-\sum_{\mathbf{j} \in \beta} c_{\mathbf{j}}^{\mathbf{b}} \cdot \mathbf{x}^{\mathbf{j}}, \quad c_{\mathbf{j}}^{\mathbf{b}} \in B,
$$

one for each $\mathbf{b} \in \mathfrak{B}(\beta)$ (the set of boundary monomials), and that these polynomials generate the ideal $J \subseteq B[\mathbf{x}]$.

Lemma 14. The quotient $B[\mathbf{x}] / J$ is generated as a $B$-module by the monomials in $\beta$.

Proof. It suffices to prove that every monomial $m$ is congruent $(\bmod J)$ to a $B$-linear combination of basis monomials. This is immediate for the basis monomials, and is also clearly true for the boundary monomials, by definition of the $G_{\mathbf{b}}$. In particular, $1 \in \beta$, the unique monomial of total degree 0 , has this property. Proceeding by induction on the total degree, suppose that every monomial of total degree less than $s$ is congruent $(\bmod J)$ to a $B$-linear combination of basis monomials, and let $m$ have total degree $s$. Let $x_{j}$ be a variable that appears in $m$, so that $m^{\prime}=m / x_{j}$ is a monomial of total degree less than $s$. Then the induction hypothesis yields

$$
\begin{aligned}
m=x_{j} \cdot m^{\prime} & \equiv x_{j} \cdot\left(\sum_{\mathbf{j} \in \beta} a_{\mathbf{j}} \cdot \mathbf{x}^{\mathbf{j}}\right) \\
& \equiv \sum_{\mathbf{j} \in \beta} a_{\mathbf{j}} \cdot\left(x_{j} \cdot \mathbf{x}^{\mathbf{j}}\right) \quad(\bmod J) .
\end{aligned}
$$

If any of the monomials $x_{j} \cdot \mathbf{x}^{\mathbf{j}}$ is not a basis monomial, then it is a boundary monomial, and can be expanded $(\bmod J)$ as a $B$-linear combination of basis monomials, showing that such a representation also obtains for $m$, as desired.

We define a pseudosyzygy of the polynomials $G_{\mathbf{b}}$ to be a $q$-tuple $\left(f_{1}, \ldots, f_{q}\right)$ of elements of $B[\mathbf{x}]$ such that

$$
\sum_{\mathbf{b} \in \mathfrak{B}(\beta)} f_{\mathbf{b}} \cdot G_{\mathbf{b}}=\left(\begin{array}{l}
\text { a } B \text {-linear combination of } \\
\text { basis monomials }
\end{array}\right) .
$$

We can construct "basic pseudosyzygies" using the syzygy-making process described in Section 4.2 (where the quotient $B[\mathbf{x}] / J$ was $B$-free with basis $\beta$ ). The 
details will be presented below, following some preliminaries on boundary monomials. Note that Lemma 13 immediately yields the following corollary.

Corollary 15. If the quotient $B[\mathbf{x}] / J$ is $B$-free with basis $\beta$, then any pseudosyzygy of the generators $G_{\mathbf{b}}$, in (14), of $J$ is in fact a syzygy of these polynomials.

5.2. Preliminaries on boundary monomials. We begin with the following simple but useful lemma.

Lemma 16. If $\mathbf{x}^{\mathbf{j}}$ is a basis monomial, then $x_{j} \cdot \mathbf{x}^{\mathbf{j}}$ is either a basis monomial or a boundary monomial, for $1 \leq j \leq n$. Furthermore, if $\mathbf{x}^{\mathbf{b}}$ is a boundary monomial that involves the variable $x_{j}$ nontrivially, then $\mathbf{x}^{\mathbf{b}} / x_{j}$ is either a boundary monomial or a basis monomial.

Proof. The first statement is immediate from the definition of boundary monomial. For the second statement, we reason as follows: If $\mathbf{x}^{\mathbf{b}}$ is a boundary monomial, then $\mathbf{x}^{\mathbf{b}} / x_{i}$ is a basis monomial for some $i$. If $i=j$, we are done; otherwise, $\mathbf{x}^{\mathbf{b}} / x_{i} \in \beta$ implies that $\mathbf{x}^{\mathbf{b}} /\left(x_{i} x_{j}\right) \in \beta$. It then follows from the first statement that $\mathbf{x}^{\mathbf{b}} / x_{j}$ is either a basis or a boundary monomial, as desired.

Next we define a relation of "adjacency" among boundary monomials. By definition, two distinct boundary monomials $\mathbf{x}^{\mathbf{b}_{1}}$ and $\mathbf{x}^{\mathbf{b}_{2}}$ are adjacent if they each differ from their least common multiple by at most multiplication by a variable, that is,

$$
\operatorname{deg}\left(\operatorname{lcm}\left(\mathbf{x}^{\mathbf{b}_{1}}, \mathbf{x}^{\mathbf{b}_{2}}\right)\right) \leq \operatorname{deg}\left(\mathbf{x}^{\mathbf{b}_{i}}\right)+1 \quad \text { for } i=1,2 .
$$

For example, the adjacent pairs among the boundary monomials shown in (12) are, up to order, $\left(x_{2}^{3}, x_{1} x_{2}^{2}\right),\left(x_{1} x_{2}^{2}, x_{1} x_{2}\right)$, and $\left(x_{1} x_{2}, x_{1}^{2}\right)$. We will see that a basic pseudosyzygy can be constructed starting with any adjacent pair of boundary monomials; indeed, we have already seen examples of this in Section 4.2.

We conclude our boundary monomial preliminaries with a partition of the set $\mathfrak{B}(\beta)$ that plays an important role in our proofs of the main results on pseudosyzygies. We first define the boundary monomial $\mathbf{x}^{\mathbf{b}}$ to be $x_{j}$-exposable provided that $\mathbf{x}^{\mathbf{b}} / x_{j}$ is a basis monomial. (The idea is that multiplication by $x_{j}$ moves the basis monomial $\mathbf{x}^{\mathbf{b}} / x_{j}$ to the "exposed" position $\mathbf{x}^{\mathbf{b}} \notin \beta$.) We then say that the boundary monomial $\mathbf{x}^{\mathbf{b}}$ is on face $i$ of $\beta$ provided that $\mathbf{x}^{\mathbf{b}}$ is $x_{i}$-exposable but not $x_{j}$-exposable for $n \geq j>i$. For example, observe that the monomial $x_{i}^{w_{i}}$ is on face $i$, where $w_{i}>0$ is the minimum exponent $e$ such that $x_{i}^{e}$ is not a basis monomial. In particular, this shows that every face is nonempty. We will call $w_{i}$ the $x_{i}$-width of the basis set $\beta$. The following result is then immediate.

Lemma 17. The set of faces of $\beta$ defines a partition of $\mathfrak{B}(\beta)$. Furthermore, if $\mathbf{x}^{\mathbf{j}}$ is a basis monomial such that $x_{j} \cdot \mathbf{x}^{\mathbf{j}}$ is a boundary monomial on face $i$, then $i \geq j$.

For example, the first and second faces of the basis set shown in (12) are, respectively, $\left\{x_{1}^{2}, x_{1} x_{2}^{2}\right\}$ and $\left\{x_{1} x_{2}, x_{2}^{3}\right\}$. 
5.3. Basic pseudosyzygies. Suppose given polynomials $G_{\mathbf{b}}$ of the form (14), one for each $\mathbf{b} \in \mathfrak{B}(\beta)$. For each adjacent pair of boundary monomials $\mathbf{b}_{1}, \mathbf{b}_{2}$, we now define a basic pseudosyzygy $P\left(\mathbf{b}_{1}, \mathbf{b}_{2}\right)$ of the polynomials $G_{\mathbf{b}}$, by repeating the sygygy-making process discussed in Section 4.2. To do this, we let

$$
z_{i}=\operatorname{lcm}\left(\mathbf{b}_{1}, \mathbf{b}_{2}\right) / \mathbf{b}_{i}, \quad \text { for } i=1,2,
$$

(each of $z_{1}, z_{2}$ is either a variable or the constant 1 , and at least one of them is a variable) and then compute

$$
-z_{1} \cdot G_{\mathbf{b}_{1}}+z_{2} \cdot G_{\mathbf{b}_{2}} .
$$

The terms $-z_{1} \cdot \mathbf{x}^{\mathbf{b}_{1}}$ and $z_{2} \cdot \mathbf{x}^{\mathbf{b}_{2}}$ cancel out, leaving behind a $B$-linear combination of basis and boundary monomials. We let $a_{\mathbf{b}} \in B$ denote the coefficient of the boundary monomial $\mathbf{x}^{\mathbf{b}}$ in (16), and observe that the expression

$$
-z_{1} \cdot G_{\mathbf{b}_{1}}+z_{2} \cdot G_{\mathbf{b}_{2}}-\sum_{\mathbf{b} \in \mathfrak{B}(\beta)} a_{\mathbf{b}} \cdot G_{\mathbf{b}}
$$

is a $B$-linear combination of basis monomials. By definition, $P\left(\mathbf{b}_{1}, \mathbf{b}_{2}\right)$ is the pseudosyzygy defined by the coefficients of (17); precisely,

$$
\begin{aligned}
P\left(\mathbf{b}_{1}, \mathbf{b}_{2}\right) & =\left(p_{\mathbf{b}}\right)_{\mathbf{b} \in \mathfrak{B}(\beta)}, \quad \text { where } \\
p_{\mathbf{b}_{1}} & =-z_{1}-a_{\mathbf{b}_{1}}, \\
p_{\mathbf{b}_{2}} & =z_{2}-a_{\mathbf{b}_{2}}, \quad \text { and } \\
p_{\mathbf{b}} & =-a_{\mathbf{b}} \quad \text { for } \mathbf{b} \neq \mathbf{b}_{1}, \mathbf{b}_{2} .
\end{aligned}
$$

We can specify the constants $a_{\mathbf{b}}$ more precisely; indeed,

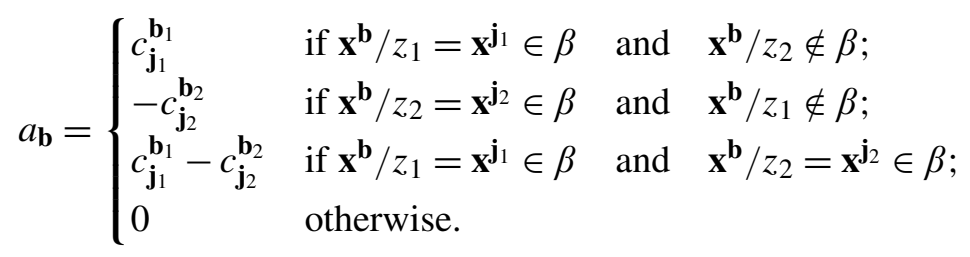

Remark 18. It is clear from the construction that the basic pseudosyzygy $P\left(\mathbf{b}_{1}, \mathbf{b}_{2}\right)$ is the unique pseudosyzygy of the form (18); that is, the constants $a_{\mathbf{b}}$ are uniquely determined. In particular, one has that $P\left(\mathbf{b}_{2}, \mathbf{b}_{1}\right)=-P\left(\mathbf{b}_{1}, \mathbf{b}_{2}\right)$.

Lemma 19. Let $x_{j}$ be the variable of minimum index $j$ in the set $\left\{z_{1}, z_{2}\right\}$. Then the constants $a_{\mathbf{b}}=0$ for all $\mathbf{x}^{\mathbf{b}}$ on faces $1,2, \ldots, j-1$.

Proof. The boundary monomials in the expression (16) are exposed by multiplication by $x_{j}$ and (possibly) by multiplication by $x_{k}$ for $k>j$, and therefore lie on faces $j$ or greater, by Lemma 17 . 
Lemma 20. Suppose that $z_{1}=1$ and $z_{2}=x_{j}$. Then for all boundary monomials $\mathbf{x}^{\mathbf{b}}$ that are not $x_{j}$-exposable, the corresponding constant $a_{\mathbf{b}}$ is zero. In particular, $a_{\mathbf{b}_{1}}=0$, or, equivalently, $p_{\mathbf{b}_{1}}=-1$.

Proof. By (19) and the hypothesis, the constant $a_{\mathbf{b}}$ can only be nonzero if $\mathbf{x}^{\mathbf{b}}$ is $x_{j}$ exposable. If $\mathbf{x}^{\mathbf{b}_{1}}$ were $x_{j}$-exposable, then $\mathbf{x}^{\mathbf{b}_{2}}=\mathbf{x}^{\mathbf{b}_{1}} / x_{j}$ would be a basis monomial, which is a contradiction.

Lemma 21. Let $\delta: B \rightarrow B^{\prime}$ be a ring homomorphism, and let $\hat{\delta}$ denote the map $B[\mathbf{x}] \rightarrow B^{\prime}[\mathbf{x}]$ induced by $\delta$. Then $\hat{\delta}\left(P\left(\mathbf{b}_{1}, \mathbf{b}_{2}\right)\right)$, obtained by applying $\hat{\delta}$ to each component of $P\left(\mathbf{b}_{1}, \mathbf{b}_{2}\right)$, is the basic pseudosyzygy of the polynomials $\hat{\delta}\left(G_{\mathbf{b}}\right)$ corresponding to the adjacent pair $\left(\mathbf{b}_{1}, \mathbf{b}_{2}\right)$.

Proof. It is clear that the polynomials $\hat{\delta}\left(G_{\mathbf{b}}\right)$ are of the form (14), with coefficients lying in $B^{\prime}$. Since $P\left(\mathbf{b}_{1}, \mathbf{b}_{2}\right) \cdot\left(G_{\mathbf{b}}\right)$ is a $B$-linear combination of basis monomials, it is clear that

$$
\hat{\delta}\left(P\left(\mathbf{b}_{1}, \mathbf{b}_{2}\right) \cdot\left(G_{\mathbf{b}}\right)\right)=\hat{\delta}\left(P\left(\mathbf{b}_{1}, \mathbf{b}_{2}\right)\right) \cdot\left(\hat{\delta}\left(G_{\mathbf{b}}\right)\right)
$$

is a $B^{\prime}$-linear combination of basis monomials. Therefore $\hat{\delta}\left(P\left(\mathbf{b}_{1}, \mathbf{b}_{2}\right)\right.$ is a pseudosyzygy having the same form as the basic pseudosyzygy of the $\hat{\delta}\left(G_{\mathbf{b}}\right)$ corresponding to the adjacent pair $\left(\mathbf{b}_{1}, \mathbf{b}_{2}\right)$, and so is equal to it by Remark 18 .

Let $J \subseteq B[\mathbf{x}]$ denote the ideal generated by the polynomials $G_{\mathbf{b}}$. By Corollary 15 , we know that if $B[\mathbf{x}] / J$ is $B$-free with basis $\beta$, then the basic pseudosyzygies $P\left(\mathbf{b}_{1}, \mathbf{b}_{2}\right)$ are syzygies of the polynomials $G_{\mathbf{b}}$. In fact, the basic (pseudo)syzygies generate the full $B[\mathbf{x}]$-module of syzygies in this case. Conversely, if the basic pseudosyzygies are syzygies, then the quotient $B[\mathbf{x}] / J$ is $B$-free with basis $\beta$. The goal of the next section is to prove these statements (in Theorem 22).

\section{Main results on pseudosyzygies}

We continue to use the notation of Section 5 . Let $\mathscr{S}$ denote the $B[\mathbf{x}]$-linear span of the basic pseudosyzygies $P\left(\mathbf{b}_{1}, \mathbf{b}_{2}\right)$ associated to a set of polynomials $G_{\mathbf{b}}$ of the form (14). The two basic technical facts demonstrated in this section can be summarized as follows.

Lemma 33. An arbitrary $q$-tuple $\left(h_{\mathbf{b}}\right)_{\mathbf{b} \in \mathfrak{B}(\beta)}$ of elements of $B[\mathbf{x}]$ can be expressed in the form

$$
\left(h_{\mathbf{b}}\right)=(\text { element of } \mathscr{S})+(\text { "reduced" tuple }) .
$$

In other words, every coset of $\mathscr{Y}$ contains at least one reduced representative.

Lemma 26. A reduced tuple that is a pseudosyzygy of the polynomials $G_{\mathbf{b}}$ must be the zero-tuple. 
Our main result (Theorem 22) on pseudosyzygies follows easily from these two lemmas. We will first state and prove the main theorem, assuming the lemmas and deferring the definition of "reduced" tuple. The proofs of the lemmas and other preliminary results are then presented. These are somewhat complicated and tedious, and can be skipped on first reading, but they rely on nothing more than polynomial arithmetic.

6.1. Main theorem on pseudosyzygies. Recall that $J \subseteq B[\mathbf{x}]$ denotes the ideal generated by the chosen set of polynomials $G_{\mathbf{b}}$, for $\mathbf{b} \in \mathfrak{B}(\beta)$.

Theorem 22. Let the hypotheses be those given at the start of Section 5.1. If the quotient $B[\mathbf{x}] / J$ is $B$-free with basis $\beta$, then the basic pseudosyzygies are syzygies that generate the entire $B[\mathbf{x}]$-module of syzygies of the polynomials $G_{\mathbf{b}}$. Conversely, if the basic pseudosyzygies of the $G_{\mathbf{b}}$ are all syzygies, then the quotient $B[\mathbf{x}] / J$ is $B$-free with basis $\beta$.

Proof. First suppose that the quotient $B[\mathbf{x}] / J$ is $B$-free with basis $\beta$. Corollary 15 implies that the basic pseudosyzygies are syzygies of the polynomials $G_{\mathbf{b}}$. We must prove that an arbitrary syzygy $\left(f_{\mathbf{b}}\right)$ of the $G_{\mathbf{b}}$ can be written as a $B[\mathbf{x}]$-linear combination of the basic pseudosyzygies, that is, $\left(f_{\mathbf{b}}\right) \in \mathscr{Y}$. However, by Lemma 33 , we know that $\left(f_{\mathbf{b}}\right)=\left(f_{\mathbf{b}}^{\prime}\right)+$ (reduced tuple), with $\left(f_{\mathbf{b}}^{\prime}\right) \in \mathscr{Y}$. It follows easily that the reduced tuple is a syzygy, and therefore a pseudosyzygy, of the $G_{\mathbf{b}}$. Lemma 26 now yields that the reduced tuple is the zero-tuple; whence, $\left(f_{\mathbf{b}}\right)=\left(f_{\mathbf{b}}^{\prime}\right) \in \mathscr{Y}$, as desired.

To prove the converse assertion, suppose now that all the basic pseudosyzygies are syzygies of the $G_{\mathbf{b}}$. By Lemma 14 , the $B$-module $B[\mathbf{x}] / J$ is generated by the basis monomials; it remains to prove that the latter are $B$-linearly independent. To this end, we assume given a $B$-linear combination of basis monomials $L$ such that $L \equiv 0(\bmod J)$. Since $L \in J$, there exists a pseudosyzygy $\left(f_{\mathbf{b}}\right)$ such that

$$
L=\left(f_{\mathbf{b}}\right) \cdot\left(G_{\mathbf{b}}\right)=\sum_{\mathbf{b} \in \mathfrak{B}(\beta)}\left(f_{\mathbf{b}} \cdot G_{\mathbf{b}}\right) .
$$

Let $\left(f_{\mathbf{b}}^{\prime}\right) \in\left(f_{\mathbf{b}}\right)+\mathscr{Y}$ be a reduced tuple, the existence of which is a consequence of Lemma 33. Since the basic pseudosyzygies are assumed to be syzygies of the $G_{\mathbf{b}}$, we have

$$
L=\left(f_{\mathbf{b}}\right) \cdot\left(G_{\mathbf{b}}\right)=\left(f_{\mathbf{b}}^{\prime}\right) \cdot\left(G_{\mathbf{b}}\right) ;
$$

that is, $\left(f_{\mathbf{b}}^{\prime}\right)$ is a reduced pseudosyzygy of the $G_{\mathbf{b}}$, and is therefore the zero-tuple, again by Lemma 26 . We conclude that $L=0$, which implies that the basis monomials are $B$-linearly independent $(\bmod J)$. This completes the proof.

Remark 23. The theorem just proved is a direct generalization of [Huibregtse 2002 , Theorem 4.3.6, p. 119] from the case of the affine plane $(n=2)$ to an affine 
space of arbitrary dimension $n$. The proof just presented is essentially the same as the proof of the cited theorem.

As noted earlier, the balance of this section is devoted to the development and proof of the lemmas needed to prove Theorem 22. The reader impatient to see how the theorem is used to construct the universal pairs $\left(U_{\beta}, Z_{\beta}\right)$ can skip to Section 7 without loss of understanding.

6.2. Extreme monomials and reduced tuples. We call a boundary monomial $\mathbf{x}^{\mathbf{b}}$ extreme if it satisfies the following condition: $\mathbf{x}^{\mathbf{b}}$ lies on face $i$ and only involves the variables $x_{1}, x_{2}, \ldots, x_{i}$ (where, of course, $x_{i}$ must be present). We say that a $q$-tuple $\left(h_{\mathbf{b}}\right)_{\mathbf{b} \in \mathfrak{B}(\beta)}$ of elements of $B[\mathbf{x}]$ is reduced if it satisfies the conditions

- When $\mathbf{x}^{\mathbf{b}}$ is extreme and on the $i$-th face, then the tuple entry $h_{\mathbf{b}}$ only involves the variables $x_{i}, x_{i+1}, \ldots, x_{n}$, and

- when $\mathbf{x}^{\mathbf{b}}$ is non-extreme, then the tuple entry $h_{\mathbf{b}}$ is 0 .

For example, consider the boundary monomials in (12). There is only one extreme monomial on face 1 , namely $x_{1}^{2}=\mathbf{x}^{\mathbf{b}_{1}}$, but both of the monomials on face 2 are extreme: $x_{1} x_{2}=\mathbf{x}^{\mathbf{b}_{2}}$ and $x_{2}^{3}=\mathbf{x}^{\mathbf{b}_{4}}$. The monomial $x_{1} x_{2}^{2}=\mathbf{x}^{\mathbf{b}_{3}}$ on face 1 is not extreme. Therefore, the following tuples $\left(h_{\mathbf{b}_{1}}, h_{\mathbf{b}_{2}}, h_{\mathbf{b}_{3}}, h_{\mathbf{b}_{4}}\right) \in(B[\mathbf{x}])^{4}$ are examples of reduced tuples:

$$
(0,0,0,0) ; \quad\left(x_{1}^{2}+x_{1} x_{2}^{2}, x_{2}^{3}, 0, x_{2}^{4}\right) ; \quad\left(1, x_{2}, 0,1\right) .
$$

Remark 24. It is clear that, in general, all monomials on face $n$ are extreme, whereas only $x_{1}^{w_{1}}$ is extreme on face 1 (recall that $w_{1}$ is the $x_{1}$-width of $\beta$, that is, $w_{1}$ is the smallest exponent $e$ such that $x_{1}^{e}$ is not a basis monomial).

6.3. Reduced pseudosyzygies. The purpose of this subsection is to prove Lemma 26 , which states that a reduced $q$-tuple that is a pseudosyzygy of the polynomials $G_{\mathbf{b}}$ must be the zero-tuple. For this we need the following lemma.

Lemma 25. Let $\mathbf{x}^{\mathbf{b}}=x_{1}^{e_{1}} x_{2}^{e_{2}} \ldots x_{j}^{e_{j}}$ be an extreme boundary monomial on face $j$ of $\beta$, and let $\mathbf{x}^{\mathbf{b}^{\prime}}$ be a boundary monomial on face $j^{\prime}>j$. Then any term in the polynomial $G_{\mathbf{b}^{\prime}}$ with monomial

$$
x_{1}^{e_{1}} x_{2}^{e_{2}} \ldots x_{j-1}^{e_{j-1}} x_{j}^{e_{j}^{\prime}} x_{j+1}^{e_{j+1}^{\prime}} \ldots x_{n}^{e_{n}^{\prime}}
$$

(that is, which has the same "prefix" as $\mathbf{x}^{\mathbf{b}}$ through position $j-1$ ) satisfies the inequality $e_{j}^{\prime}<e_{j}$. In particular, any basis monomial $\mathbf{x}^{\mathbf{j}}$ with the same prefix as $\mathbf{x}^{\mathbf{b}}$ through position $j-1$ satisfies this inequality.

Proof. First of all, note that if there were a basis monomial having the prefix $x_{1}^{e_{1}} \ldots x_{j-1}^{e_{j-1}} x_{j}^{e_{j}^{\prime}}$ and $e_{j}^{\prime} \geq e_{j}$, then $\mathbf{x}^{\mathbf{b}}$ would divide a basis monomial, which implies 
that $\mathbf{x}^{\mathbf{b}}$ would be a basis monomial, which is a contradiction. So, if the polynomial $G_{\mathbf{b}^{\prime}}$ is to have a term with prefix $x_{1}^{e_{1}} x_{2}^{e_{2}} \ldots x_{j-1}^{e_{j-1}} x_{j}^{e_{j}^{\prime}}$ with $e_{j}^{\prime} \geq e_{j}$, this term must be the boundary monomial $\mathbf{x}^{\mathbf{b}^{\prime}}$. However, this monomial lies on face $j^{\prime}>j$, by hypothesis; therefore, $\mathbf{x}^{\mathbf{b}^{\prime}} / x_{j^{\prime}}$ is a basis monomial that $\mathbf{x}^{\mathbf{b}}$ divides, so we again obtain a contradiction. It follows that $e_{j}^{\prime}<e_{j}$ must hold, as asserted.

We may now prove the following lemma.

Lemma 26. If $\left(h_{\mathbf{b}}\right)_{\mathbf{b} \in \mathfrak{B}(\beta)}$ is reduced and is a pseudosyzygy of the polynomials $G_{\mathbf{b}}$, then $\left(h_{\mathbf{b}}\right)$ is the zero-tuple.

Proof. By definition, it suffices to prove that all of the tuple components at extreme positions are 0 . We begin with the first face. There is only one extreme monomial on face 1 , namely $x_{1}^{w_{1}}=\mathbf{x}^{\mathbf{b}_{1}}$. Now, the component $h_{\mathbf{b}_{1}}$ can involve all of the variables, but the definition of reduced tuple implies that this is the only component that can involve $x_{1}$ nontrivially. Write $h_{\mathbf{b}_{1}}$ as a polynomial in $x_{1}$ with coefficients in $B\left[x_{2}, \ldots, x_{n}\right]$, and let $a \cdot x_{1}^{t}$ be the term of highest degree. We claim that in the dot product $\left(h_{\mathbf{b}}\right) \cdot\left(G_{\mathbf{b}}\right)$, the term $a \cdot x_{1}^{\left(t+w_{1}\right)}$ coming from

\section{(highest degree term of $h_{\mathbf{b}_{1}}$ ) $\left(\right.$ (boundary monomial of $G_{\mathbf{b}_{1}}$ )}

cannot cancel out. Then, since $x_{1}^{\left(t+w_{1}\right)}$ is not a basis monomial, $\left(h_{\mathbf{b}}\right)$ can't be a pseudosyzygy unless $a=0$, which implies that the component $h_{\mathbf{b}_{1}}=0$. To prove the claim, we note that a cancelling term can only come from $h_{\mathbf{b}^{\prime}} \cdot G_{\mathbf{b}^{\prime}}$ for $\mathbf{x}^{\mathbf{b}^{\prime}}$ an extreme monomial on face $j \geq 1$. If $j>1$, we know by definition of reduced tuple that $h_{\mathbf{b}^{\prime}}$ only involves the variables $x_{j}, \ldots, x_{n}$, and so does not involve $x_{1}$. We therefore cannot produce a cancelling term containing the monomial $x_{1}^{\left(t+w_{1}\right)}$ from $h_{\mathbf{b}}^{\prime} \cdot G_{\mathbf{b}^{\prime}}$, because the polynomial $G_{\mathbf{b}^{\prime}}$ can only involve $x_{1}$ to powers at most $w_{1}-1$, by the preceding lemma. If $j=1$, then a cancelling term can only arise from a product of the form $h_{\mathbf{b}_{1}} \cdot c_{\mathbf{j}}^{\mathbf{b}_{1}} \mathbf{x}^{\mathbf{j}}$ for some $\mathbf{j} \in \beta$ (recall (14) for the notation). However, the preceding lemma implies that the $x_{1}$-degree of $\mathbf{x}^{\mathbf{j}}$ is $\leq w_{1}-1$, and since the maximal $x_{1}$-degree of $h_{\mathbf{b}_{1}}$ is $t$, we again cannot produce a term of $x_{1}$-degree $t+w_{1}$. This completes the proof of the claim.

Working now by induction on $j$, suppose that all the tuple entries $h_{\mathbf{b}}$ associated to extreme monomials $\mathbf{x}^{\mathbf{b}}$ on faces $1, \ldots, j-1$ have been shown to be zero. Consider the extreme monomials on face $j$ : these are the boundary monomials $\mathbf{x}^{\mathbf{b}_{1}}, \mathbf{x}^{\mathbf{b}_{2}}, \ldots, \mathbf{x}^{\mathbf{b}_{r}}$ that involve only the variables $x_{1}, x_{2}, \ldots, x_{j}$, and such that $\mathbf{x}^{\mathbf{b}_{i}} / x_{j}$ is a basis monomial for $i=1,2, \ldots, r$. The corresponding tuple components $h_{\mathbf{b}_{i}}$ can only involve $x_{j}, x_{j+1}, \ldots x_{n}$ nontrivially; moreover, these are the only components that can involve $x_{j}$ nontrivially (a consequence of the definition of a reduced tuple and the induction hypothesis). Let $t$ be the maximum exponent of $x_{j}$ that occurs among the nonzero $h_{\mathbf{b}_{i}}$, and suppose without loss of generality that 
$x_{j}^{t}$ occurs in $h_{\mathbf{b}_{1}}$. Let

$$
h_{\mathbf{b}_{1}}=a \cdot x_{j}^{t}+\left(\text { terms with lower } x_{j}\right. \text {-degree), }
$$

where $a \in B\left[x_{j+1}, \ldots, x_{n}\right]$. Now, the boundary monomial of $G_{\mathbf{b}_{1}}$, namely $\mathbf{x}^{\mathbf{b}_{1}}$, only involves the variables $x_{1}, \ldots, x_{j}$; say $\mathbf{x}^{\mathbf{b}_{1}}=x_{1}^{e_{1}} x_{2}^{e_{2}} \ldots x_{j}^{e_{j}}$. When the boundary monomial of $G_{\mathbf{b}_{1}}$ is multiplied by $h_{\mathbf{b}_{1}}$ (in computing the dot product $\left(h_{\mathbf{b}}\right) \cdot\left(G_{\mathbf{b}}\right)$ ), the terms

$$
a \cdot x_{1}^{e_{1}} \ldots x_{(j-1)}^{e_{(j-1)}} \cdot x_{j}^{\left(e_{j}+t\right)} \quad \text { where } a \in B\left[x_{j+1}, \ldots, x_{n}\right]
$$

are produced, and these terms must cancel out if the tuple $\left(h_{\mathbf{b}}\right)$ is to be a pseudosyzygy.

We first claim that these terms cannot cancel with any terms produced by the products $h_{\mathbf{b}^{\prime}} \cdot G_{\mathbf{b}^{\prime}}$ corresponding to extreme monomials $\mathbf{x}^{\mathbf{b}^{\prime}}$ on faces $j+1, \ldots, n$. To see this, recall that,Lemma 25 implies that any term of $G_{\mathbf{b}^{\prime}}$ with monomial "prefix" $x_{1}^{e_{1}} x_{2}^{e_{2}} \ldots, x_{j-1}^{e_{j-1}} x_{j}^{e_{j}^{\prime}}$ must have $e_{j}^{\prime}<e_{j}$. Moreover, the coefficients $h_{\mathbf{b}^{\prime}}$ (with $\mathbf{x}^{\mathbf{b}^{\prime}}$ on some face $k>j$ ) can only involve variables in the range $x_{k}, \ldots, x_{n}$. A moment's reflection shows that the only terms with monomial prefix $x_{1}^{e_{1}} \ldots x_{(j-1)}^{e_{(j-1)}}$ that occur in $h_{\mathbf{b}^{\prime}} \cdot G_{\mathbf{b}^{\prime}}$ will have $x_{j}$-exponent $<e_{j}+t$, which proves the claim.

We next claim that the terms (23) cannot cancel with any other terms in the product $h_{\mathbf{b}_{1}} \cdot G_{\mathbf{b}_{1}}$, that is, terms of the form $\left(c_{\mathbf{j}}^{\mathbf{b}_{1}} \mathbf{x}^{\mathbf{j}}\right) \cdot h_{\mathbf{b}_{1}}$. Since $h_{\mathbf{b}_{1}}$ only involves the variables $x_{j}, x_{j+1}, \ldots, x_{n}$, with $t$ the maximum $x_{j}$-degree, in order for the latter terms to cancel with the former, the basis monomial $\mathbf{x}^{\mathbf{j}}$ would have to have the monomial prefix $x_{1}^{e_{1}} x_{2}^{e_{2}} \ldots, x_{j-1}^{e_{j-1}} x_{j}^{e_{j}^{\prime}}$ with $e_{j}^{\prime} \geq e_{j}$. However, Lemma 25 implies that $e_{j}^{\prime}<e_{j}$.

Therefore, the terms (23) can only cancel with like terms generated by the products $h_{\mathbf{b}_{i}} \cdot G_{\mathbf{b}_{i}}$ for $1<i \leq k$. Note that the various extreme monomials $\mathbf{x}^{\mathbf{b}_{i}}$ on face $j$ must all differ from one another in the variables $x_{1}, \ldots, x_{(j-1)}$, since otherwise two distinct monomials on face $j$ would differ only by a power of $x_{j}$, but this clearly cannot occur. Hence, since none of the $h_{\mathbf{b}_{i}}$ involve $x_{1}, \ldots, x_{j-1}$, we see that the terms produced by the products $h_{\mathbf{b}_{i}} \cdot \mathbf{x}^{\mathbf{b}_{i}}$ cannot cancel with the terms (23). The last hope for cancelling these terms lies in the products of the form $h_{\mathbf{b}_{i}} \cdot\left(c_{\mathbf{j}}^{\mathbf{b}_{i}} \mathbf{x}^{\mathbf{j}}\right)$. However, since $t$ was chosen as the maximum $x_{j}$ degree among all the $h_{\mathbf{b}_{i}}$, we can repeat the reasoning of the preceding paragraph to see that such cancellation is impossible. We conclude that $h_{\mathbf{b}_{i}}=0$ for $1 \leq i \leq r$, which completes the induction step and the proof of the lemma.

Having proved Lemma 26, we now proceed to the proof of Lemma 33, which states that an arbitrary $q$-tuple $\left(h_{\mathbf{b}}\right)_{\mathbf{b} \in \mathfrak{B}(\beta)}$ can be written as the sum of an element of $\mathscr{Y}$ and a reduced tuple, as in (20). We accomplish this goal by repeated application of certain $q$-tuple rewriting steps called "degree lowering" and "column clearing," which we proceed to discuss. 
6.4. Degree lowering. We say that the basic pseudosyzygy $P\left(\mathbf{b}_{1}, \mathbf{b}_{2}\right)$ lowers $x_{j}$ degree at $\mathbf{b}_{1}$ provided that (recall (18))

$$
p_{\mathbf{b}_{1}}=-x_{j}-a_{\mathbf{b}_{1}} .
$$

The reason for the terminology is the following: Suppose that we have an arbitrary $q$-tuple $\left(h_{\mathbf{b}}\right)_{\mathbf{b} \in \mathfrak{B}(\beta)}$ of elements of $B[\mathbf{x}]$, and that the maximum $x_{j}$-degree of the component $h_{\mathbf{b}_{1}}$ is $t>0$. Write

$$
h_{\mathbf{b}_{1}}=\gamma \cdot x_{j}^{t}+\left(\text { lower } x_{j} \text {-degree terms), } \quad \gamma \in B\left[x_{1}, \ldots, x_{j-1}, x_{j+1}, \ldots, x_{n}\right] .\right.
$$

Then

$$
\left(h_{\mathbf{b}}\right)=-\gamma \cdot x_{j}^{(t-1)} \cdot P\left(\mathbf{b}_{1}, \mathbf{b}_{2}\right)+\left(\left(h_{\mathbf{b}}\right)+\gamma \cdot x_{j}^{(t-1)} \cdot P\left(\mathbf{b}_{1}, \mathbf{b}_{2}\right)\right),
$$

which shows that, modulo the $B[\mathbf{x}]$-linear span of the basic pseudosyzygies $\mathscr{Y}$, the coset of $\left(h_{\mathbf{b}}\right)$ contains a representative

$$
\left(h_{\mathbf{b}}^{\prime}\right)=\left(h_{\mathbf{b}}\right)+\gamma \cdot x_{j}^{(t-1)} \cdot P\left(\mathbf{b}_{1}, \mathbf{b}_{2}\right)
$$

such that the $x_{j}$-degree of component $h_{\mathbf{b}_{1}}^{\prime}$ is $\leq t-1$.

Lemma 27. If the boundary monomial $\mathbf{x}^{\mathbf{b}_{1}}$ is $x_{i}$-exposable, then for all variables $x_{j} \neq x_{i}$, there is a basic pseudosyzygy $P\left(\mathbf{b}_{1}, \mathbf{b}_{2}\right)$ that lowers $x_{j}$-degree at $\mathbf{b}_{1}$. For $\mathbf{b} \neq \mathbf{b}_{1}$, the components $h_{\mathbf{b}}^{\prime}$ of the resulting $q$-tuple differ from the corresponding components $h_{\mathbf{b}}$ of the original $q$-tuple only by terms of $x_{j}$-degree $t-1$, where $t$ is the maximum $x_{j}$-degree of the component $h_{\mathbf{b}_{1}}$. Furthermore, if $\mathbf{x}^{\mathbf{b}_{1}}$ is on face $i$, and $j<i$, then we can choose $P\left(\mathbf{b}_{1}, \mathbf{b}_{2}\right)$ to lower $x_{j}$-degree at $\mathbf{b}_{1}$ and to ensure that the components $h_{\mathbf{b}}$ and $h_{\mathbf{b}}^{\prime}$ are equal for all $\mathbf{x}^{\mathbf{b}}$ on faces $1,2, \ldots, j-1$.

Proof. If $\mathbf{x}^{\mathbf{b}_{1}}$ is $x_{i}$-exposable, then $\mathbf{x}^{\mathbf{b}_{1}} / x_{i}=m$ is a basis monomial. If $x_{j} \cdot m$ is a basis monomial, then we must have that $\left(x_{j} \cdot m\right) \cdot x_{i}=\mathbf{x}^{\mathbf{b}_{2}}$ is a boundary monomial. (If not, it would be a basis monomial, but then by definition its divisor $m \cdot x_{i}=\mathbf{x}^{\mathbf{b}_{1}}$ would have to be a basis monomial, which is a contradiction.) The only other possibility is that $x_{j} \cdot m=\mathbf{x}^{\mathbf{b}_{2}}$ is a boundary monomial. In either case, one sees easily that $\left(\mathbf{b}_{1}, \mathbf{b}_{2}\right)$ is an adjacent pair such that

$$
\begin{aligned}
& z_{1}=\operatorname{lcm}\left(\mathbf{b}_{1}, \mathbf{b}_{2}\right) / \mathbf{b}_{1}=x_{j}, \\
& z_{2}=\operatorname{lcm}\left(\mathbf{b}_{1}, \mathbf{b}_{2}\right) / \mathbf{b}_{2}= \begin{cases}1 & \text { if } x_{j} \cdot m \text { is a basis monomial } \\
x_{i} & \text { if } x_{j} \cdot m \text { is not a basis monomial. }\end{cases}
\end{aligned}
$$

By definition, the basic pseudosyzygy $P\left(\mathbf{b}_{1}, \mathbf{b}_{2}\right)$ lowers $x_{j}$-degree at $\mathbf{b}_{1}$. This proves the first statement.

The second statement follows from the observation that

$$
h_{\mathbf{b}}^{\prime}=h_{\mathbf{b}}+\gamma \cdot x_{j}^{(t-1)} \cdot p_{\mathbf{b}}
$$


(recall (18) and (25)) where, for $\mathbf{b} \neq \mathbf{b}_{1}$, we have that $p_{\mathbf{b}}$ is either a constant (in $B$ ) or a linear polynomial over $B$ involving a variable other than $x_{j}$.

To prove the last statement we will show that the basic pseudosyzygy $P\left(\mathbf{b}_{1}, \mathbf{b}_{2}\right)$ constructed above has the desired property; for this it suffices to prove that the component $p_{\mathbf{b}}$ in equation (26) vanishes for $\mathbf{x}^{\mathbf{b}}$ on face $1,2, \ldots, j-1$. However, since $\mathbf{x}^{\mathbf{b}_{1}}$ and $\mathbf{x}^{\mathbf{b}_{2}}$ lie on faces $j$ or higher, and the associated components $p_{\mathbf{b}_{1}}, p_{\mathbf{b}_{2}}$ of $P\left(\mathbf{b}_{1}, \mathbf{b}_{2}\right)$ are the only ones that can involve a variable, we have that for any $\mathbf{x}^{\mathbf{b}}$ on face $j-1$ or lower, the component $p_{\mathbf{b}}$ in equation (26) is equal to the constant $-a_{\mathbf{b}}$, which is zero by Lemma 19 .

6.5. Column clearing. We begin by partitioning the non-extreme boundary monomials of $\beta$ into subsets called "columns." Let $\mathbf{x}^{\mathbf{b}}$ be a non-extreme boundary monomial belonging to face $i$, for $1 \leq i<n$ (recall from Remark 24 that all monomials on face $n$ are extreme). Since $\mathbf{x}^{\mathbf{b}}$ is not extreme, it must involve at least one of the variables $x_{i+1}, \ldots, x_{n}$ nontrivially; let $j$ denote the smallest index greater than $i$ such that $x_{j}$ divides $\mathbf{x}^{\mathbf{b}}$. Then the column containing $\mathbf{x}^{\mathbf{b}}$ consists of all the nonextreme monomials on face $i$ that are divisible by $x_{j}$ and can be obtained from $\mathbf{x}^{\mathbf{b}}$ by multiplying or dividing by powers of $x_{j}$. We say that this column is in the $x_{j}$-direction.

Lemma 28. The members of the column containing $\mathbf{x}^{\mathbf{b}}$ are

$$
\mathbf{x}^{\mathbf{b}} \cdot x_{j}^{-s}, \mathbf{x}^{\mathbf{b}} \cdot x_{j}^{-s+1}, \ldots, \mathbf{x}^{\mathbf{b}} \cdot x_{j}^{-1}, \mathbf{x}^{\mathbf{b}}, \mathbf{x}^{\mathbf{b}} \cdot x_{j}, \mathbf{x}^{\mathbf{b}} \cdot x_{j}^{2}, \ldots, \mathbf{x}^{\mathbf{b}} \cdot x_{j}^{t}
$$

where $s, t$ are nonnegative integers, and all the listed monomials are boundary monomials on face $i$ that are divisible by $x_{j}$ but not divisible by any $x_{v}$ for $i<v<j$. Furthermore, $\mathbf{x}^{\mathbf{b}} \cdot x_{j}^{t+1}$ (which clearly cannot be a basis monomial) is either not a boundary monomial or else is a boundary monomial on face $l$ for some $l<i$, and $\mathbf{x}^{\mathbf{b}} \cdot x_{j}^{-s-1}$ is a boundary monomial (called the lower bound of the column) such that exactly one of the following holds:

- $\mathbf{x}^{\mathbf{b}} \cdot x_{j}^{-s-1}$ is an extreme monomial on face $i$;

- $\mathbf{x}^{\mathbf{b}} \cdot x_{j}^{-s-1}$ is a non-extreme monomial on face $i$ that is not divisible by $x_{j}$, and so belongs to a column in the $x_{j^{\prime}}$-direction for some $j^{\prime}>j$;

- $\mathbf{x}^{\mathbf{b}} \cdot x_{j}^{-s-1}$ is on face $k$ for some $k$ satisfying $j \leq k \leq n$.

Proof. The first statement being immediate from the definition, we turn to the second. Suppose that $\mathbf{x}^{\mathbf{b}} \cdot x_{j}^{t+1}$ is a boundary monomial. If it were on face $i$, then it would belong to the listed column, which it does not. If it were on face $k>i$, then $\left(\mathbf{x}^{\mathbf{b}} \cdot x_{j}^{t+1}\right) / x_{k}$ would be a basis monomial, hence $\left(\mathbf{x}^{\mathbf{b}} \cdot x_{j}^{t+1}\right) /\left(x_{k} x_{j}\right)=\left(\mathbf{x}^{\mathbf{b}} \cdot x_{j}^{t}\right) / x_{k}$ would be too, and we would conclude that $\mathbf{x}^{\mathbf{b}} \cdot x_{j}^{t}$ is $x_{k}$-exposable for $k>i$, a contradiction. It follows that if $\mathbf{x}^{\mathbf{b}} \cdot x_{j}^{t+1}$ is a boundary monomial, then it lies on face $l<i$, as the second statement asserts. 
To prove the last statement, we first claim that $\mathbf{x}^{\mathbf{b}} \cdot x_{j}^{-s-1}=m$ is a boundary monomial. Indeed, by Lemma $16, m=\mathbf{x}^{\mathbf{b}} \cdot x_{j}^{-s} / x_{j}$ is either a basis or a boundary monomial, but if the former were true, then $\mathbf{x}^{\mathbf{b}} \cdot x_{j}^{-s}$ would be $x_{j}$-exposable for $j>i$, a contradiction. Furthermore, since $m=x_{i} \cdot\left(\left(\mathbf{x}^{\mathbf{b}} \cdot x_{j}^{-s}\right) /\left(x_{i} x_{j}\right)\right)$, it follows that $m$ is $x_{i}$-exposable, and so lies on face $k \geq i$. In addition, $m$ is not divisible by any $x_{v}$ for $i<v<j$, since otherwise all of its multiples (including $\mathbf{x}^{\mathbf{b}}$ ) would be divisible by $x_{v}$, contradicting the hypothesis that the column of $\mathbf{x}^{\mathbf{b}}$ is in the $x_{j}$ direction. Suppose now that $m$ lies on face $k=i$. If $m$ is extreme, we have the first bulleted possibility. If $m$ is not extreme, then it cannot be divisible by $x_{j}$-if it were, then it would belong to the column of $\mathbf{x}^{\mathbf{b}}$, which is a contradiction - nor can it be divisible by $x_{v}$ for $i<v<j$, as already noted. Therefore, $m$ 's column would be in the $x_{j^{\prime}}$-direction for some $j^{\prime}>j$, and we have the second bulleted possibility. The only other possibility is that $m$ lies on face $k>i$. Since again $m$ is not divisible by any $x_{v}$ for $i<v<j$, we see that in fact $k \geq j$ must hold, which is the third bulleted possibility. This completes the proof of the lemma.

For example, there is only one non-extreme monomial in diagram (12), namely, $\mathbf{x}^{\mathbf{b}}=x_{1} x_{2}^{2}$, which is on face $i=1$. Hence there is only one column, consisting of the single monomial $x_{1} x_{2}^{2}$, that is in the $x_{j}=x_{2}$-direction. In this case, $s=t=0$, the monomial $\mathbf{x}^{\mathbf{b}} \cdot x_{2}^{t+1}=x_{1} x_{2}^{3}$ is not a boundary monomial, and $\mathbf{x}^{\mathbf{b}} \cdot x_{2}^{-s-1}=x_{1} x_{2}$ is on face $2=j$.

We say that the basic syzygy $P\left(\mathbf{b}_{1}, \mathbf{b}_{2}\right)=\left(p_{\mathbf{b}}\right)_{\mathbf{b} \in \mathfrak{B}(\beta)}$ clears the $\mathbf{b}_{1}$-entry provided that

$$
p_{\mathbf{b}_{1}}=-1 \quad \text { and } \quad p_{\mathbf{b}_{2}}=x_{j}-a_{\mathbf{b}_{2}}
$$

for some $x_{j}$ (recall that $z_{1}=1$ implies that $p_{\mathbf{b}_{1}}=-1$ by Lemma 20). The reason for the terminology is the following: Suppose that we have a $q$-tuple $\left(h_{\mathbf{b}}\right)_{\mathbf{b} \in \mathfrak{B}(\beta)}$. Then we may write

$$
\left(h_{\mathbf{b}}\right)=-h_{\mathbf{b}_{1}} \cdot P\left(\mathbf{b}_{1}, \mathbf{b}_{2}\right)+\left(\left(h_{\mathbf{b}}\right)+h_{\mathbf{b}_{1}} \cdot P\left(\mathbf{b}_{1}, \mathbf{b}_{2}\right)\right),
$$

which shows that, modulo the $B[\mathbf{x}]$-linear span of the basic pseudosyzygies $\mathscr{Y}$, the coset of $\left(h_{\mathbf{b}}\right)$ contains a representative

$$
\left(h_{\mathbf{b}}^{\prime}\right)=\left(h_{\mathbf{b}}\right)+h_{\mathbf{b}_{1}} \cdot P\left(\mathbf{b}_{1}, \mathbf{b}_{2}\right)
$$

such that $h_{\mathbf{b}_{1}}^{\prime}=0$.

The basic idea of column clearing is to compose a series of these operations to clear the components of a $q$-tuple $\left(h_{\mathbf{b}}\right)$ that correspond to an entire column of boundary monomials. In fact, by clearing columns in the right order, we can clear all of the non-extreme positions in the $q$-tuple $\left(h_{\mathbf{b}}\right)$ that correspond to monomials on faces $i$ and higher without disturbing any of the positions corresponding to monomials on faces lower than $i$. The details are given in the following lemmas. 
Lemma 29. Let $\left(h_{\mathbf{b}}\right)_{\mathbf{b} \in \mathfrak{B}(\beta)}$ be an arbitrary $q$-tuple of elements of $B[\mathbf{x}]$, and $\mathbf{x}^{\mathbf{b}_{0}}$ a non-extreme boundary monomial on face $i$, for $1 \leq i<n$, whose column is in the $x_{j}$-direction, where $i<j \leq n$. Then the coset $\left(h_{\mathbf{b}}\right)+\mathscr{Y}$ contains a $q$-tuple $\left(h_{\mathbf{b}}^{\prime}\right)$ such that $h_{\mathbf{b}}^{\prime}=0$ for all $\mathbf{x}^{\mathbf{b}}$ in the column of $\mathbf{x}^{\mathbf{b}_{0}}$, and $h_{\mathbf{b}}=h_{\mathbf{b}}^{\prime}$ for all $\mathbf{x}^{\mathbf{b}}$ on faces $1,2, \ldots, j-1$ except for those in the column of $\mathbf{x}^{\mathbf{b}_{0}}$ and the column's lower bound $\mathbf{x}^{\mathbf{b}_{0}} \cdot x_{j}^{-s-1}$, if the latter is on face $i$. (Recall from Lemma 28 that the lower bound of the column will either lie on face $i$ or on some face $k \geq j$.) In addition, for $x_{j^{\prime}} \neq x_{j}$, the differences $h_{\mathbf{b}}^{\prime}-h_{\mathbf{b}}$ have $x_{j^{\prime}}$-degree at most equal to the maximum $x_{j^{\prime}}$-degree among the components $h_{\mathbf{b}_{k}}$ corresponding to monomials $\mathbf{x}^{\mathbf{b}_{k}}$ in the cleared column. Proof. Using the notation of Lemma 28, we let $\mathbf{x}^{\mathbf{b}_{k}}=\mathbf{x}^{\mathbf{b}_{0}} \cdot x_{j}^{t-(k-1)}$ for $1 \leq k \leq$ $t+s+2$, so $\mathbf{x}^{\mathbf{b}_{1}}=\mathbf{x}^{\mathbf{b}_{0}} \cdot x_{j}^{t}$ is the "top" of the column, $\mathbf{x}^{\mathbf{b}_{t+s+2}}=\mathbf{x}^{\mathbf{b}_{0}} \cdot x_{j}^{-s-1}$ is the lower bound of the column, and the remaining $\mathbf{x}^{\mathbf{b}_{l}}$ are intermediate members of the column. Beginning at the top of the column, we note that $\mathbf{x}^{\mathbf{b}_{1}}$ and $\mathbf{x}^{\mathbf{b}_{2}}$ are an adjacent pair of boundary monomials such that

$$
z_{1}=\operatorname{lcm}\left(\mathbf{x}^{\mathbf{b}_{1}}, \mathbf{x}^{\mathbf{b}_{2}}\right) / \mathbf{x}^{\mathbf{b}_{1}}=1 \quad \text { and } \quad z_{2}=\operatorname{lcm}\left(\mathbf{x}^{\mathbf{b}_{1}}, \mathbf{x}^{\mathbf{b}_{2}}\right) / \mathbf{x}^{\mathbf{b}_{2}}=x_{j},
$$

so the basic pseudosyzygy $P\left(\mathbf{b}_{1}, \mathbf{b}_{2}\right)$ clears the $\mathbf{b}_{1}$-entry; that is, the $\mathbf{b}_{1}$-entry in the $q$-tuple

$$
\left(h_{\mathbf{b}}^{1}\right)=\left(h_{\mathbf{b}}\right)+h_{\mathbf{b}_{1}} \cdot P\left(\mathbf{b}_{1}, \mathbf{b}_{2}\right)
$$

is zero. Moreover, the constants $a_{\mathbf{b}}$ (18) corresponding to $\mathbf{x}^{\mathbf{b}}$ in faces $1,2, \ldots, j-1$ are all zero by Lemma 19. Therefore, since the entries $p_{\mathbf{b}}$ of $P\left(\mathbf{b}_{1}, \mathbf{b}_{2}\right)$ are equal to the corresponding $a_{\mathbf{b}}$ except at $\mathbf{b}_{1}$ and $\mathbf{b}_{2}$, we conclude that $h_{\mathbf{b}}^{1}=h_{\mathbf{b}}$ for all $\mathbf{x}^{\mathbf{b}}$ on faces $1,2, \ldots, j-1$, except for $\mathbf{x}^{\mathbf{b}_{1}}$ and $\mathbf{x}^{\mathbf{b}_{2}}$, if the latter lies on face $i$. (Note that if $\mathbf{x}^{\mathbf{b}_{2}}$ is not on face $i$, then it is the lower bound of the column and lies on some face $k \geq j$, by Lemma 28.) Since

$$
h_{\mathbf{b}}^{1}-h_{\mathbf{b}}=h_{\mathbf{b}_{1}} \cdot p_{\mathbf{b}},
$$

where $p_{\mathbf{b}}$ is either a constant (possibly 0 ) or, when $\mathbf{b}=\mathbf{b}_{2}$, a linear polynomial in $x_{j}$, we have that the $x_{j^{\prime}}$-degree of this difference is at most equal to the $x_{j^{\prime}}$-degree of $h_{\mathbf{b}_{1}}$, for $x_{j^{\prime}} \neq x_{j}$.

If the column consists of only one monomial $\mathbf{x}^{\mathbf{b}_{1}}$ (that is, $s=t=0$ ), then we are done. Otherwise, we proceed by induction. Suppose that for some $l$, where $1<l<t+s+2$, we have constructed a $q$-tuple $\left(h_{\mathbf{b}}^{l-1}\right) \in\left(h_{\mathbf{b}}\right)+\mathscr{Y}$ such that

$$
\begin{aligned}
& h_{\mathbf{b}_{1}}^{l-1}=h_{\mathbf{b}_{2}}^{l-1}=\cdots=h_{\mathbf{b}_{l-1}}^{l-1}=0, \\
& h_{\mathbf{b}}^{l-1}=h_{\mathbf{b}}
\end{aligned}
$$

for all $\mathbf{x}^{\mathbf{b}}$ on faces $1,2, \ldots, j-1$, except for $\mathbf{x}^{\mathbf{b}_{1}}, \mathbf{x}^{\mathbf{b}_{2}}, \ldots, \mathbf{x}^{\mathbf{b}_{l}}$ (all of which are in the column of $\mathbf{x}^{\mathbf{b}_{0}}$, and hence on face $i$ ); and the differences $h_{\mathbf{b}}^{l-1}-h_{\mathbf{b}}$ have maximum $x_{j^{\prime}}$-degree at most equal to the maximum $x_{j^{\prime}}$-degree among the components 
$h_{\mathbf{b}_{1}}, h_{\mathbf{b}_{2}}, \ldots, h_{\mathbf{b}_{l-1}}$. We then use the basic pseudosyzygy $P\left(\mathbf{b}_{l}, \mathbf{b}_{l+1}\right)$ to clear the $\mathbf{b}_{l}$-entry of the tuple $\left(h_{\mathbf{b}}^{l-1}\right)$ and obtain the tuple

$$
\left(h_{\mathbf{b}}^{l}\right)=\left(h_{\mathbf{b}}^{l-1}\right)+h_{\mathbf{b}_{l}}^{l-1} \cdot P\left(\mathbf{b}_{l}, \mathbf{b}_{l+1}\right),
$$

in which $h_{\mathbf{b}_{l}}^{l}=0$. Since the entries $p_{\mathbf{b}}$ of $P\left(\mathbf{b}_{l}, \mathbf{b}_{l+1}\right)$ are equal to the corresponding $a_{\mathbf{b}}$ except at $\mathbf{b}_{l}$ and $\mathbf{b}_{l+1}$, we find as before that $h_{\mathbf{b}}^{l}=h_{\mathbf{b}}^{l-1}$ for all $\mathbf{x}^{\mathbf{b}}$ on faces $1,2, \ldots, j-1$, except for $\mathbf{x}^{\mathbf{b}_{l}}$ and $\mathbf{x}^{\mathbf{b}_{l+1}}$, if the latter is on face $i$. In view of the induction hypothesis, this implies that

$$
\begin{aligned}
h_{\mathbf{b}_{1}}^{l} & =h_{\mathbf{b}_{2}}^{l}=\cdots=h_{\mathbf{b}_{l-1}}^{l}=h_{\mathbf{b}_{l}}^{l}=0, \\
h_{\mathbf{b}}^{l} & =h_{\mathbf{b}}
\end{aligned}
$$

for all $\mathbf{x}^{\mathbf{b}}$ on faces $1, \ldots, j-1$ except for $\mathbf{x}^{\mathbf{b}_{1}}, \ldots, \mathbf{x}^{\mathbf{b}_{l}}$, and for $\mathbf{x}^{\mathbf{b}_{l+1}}$ if the latter is on face $i$ (it might not be if it were the lower bound of the column). As before, the differences $h_{\mathbf{b}}^{l}-h_{\mathbf{b}}^{l-1}$ have $x_{j^{\prime}}$-degree at most equal to the $x_{j^{\prime}}$-degree of

$$
h_{\mathbf{b}_{l}}^{l-1}=h_{\mathbf{b}_{l}}+\left(h_{\mathbf{b}_{l}}^{l-1}-h_{\mathbf{b}_{l}}\right) \text {. }
$$

Since the $x_{j^{\prime}}$-degree of the difference $h_{\mathbf{b}_{l}}^{l-1}-h_{\mathbf{b}_{l}}$ is at most equal to the maximum $x_{j^{\prime}}$-degree among the components $h_{\mathbf{b}_{1}}, h_{\mathbf{b}_{2}}, \ldots, h_{\mathbf{b}_{l-1}}$, by the induction hypothesis, we see that the differences $h_{\mathbf{b}}^{l}-h_{\mathbf{b}}^{l-1}$ have maximum $x_{j^{\prime}}$-degree at most equal to the maximum $x_{j^{\prime}}$-degree among the components $h_{\mathbf{b}_{1}}, h_{\mathbf{b}_{2}}, \ldots, h_{\mathbf{b}_{l}}$. It follows that the same estimate applies to the differences

$$
h_{\mathbf{b}}^{l}-h_{\mathbf{b}}=\left(h_{\mathbf{b}}^{l}-h_{\mathbf{b}}^{l-1}\right)+\left(h_{\mathbf{b}}^{l-1}-h_{\mathbf{b}}\right),
$$

since, by the induction hypothesis, the $x_{j^{\prime}}$-degree of the second summand is at most equal to the maximum $x_{j^{\prime}}$-degree among the components $h_{\mathbf{b}_{1}}, h_{\mathbf{b}_{2}}, \ldots, h_{\mathbf{b}_{l-1}}$. It now follows that the desired coset representative is obtained after the entire column is cleared, that is, $\left(h_{\mathbf{b}}^{\prime}\right)=\left(h_{\mathbf{b}}^{t+s+1}\right)$.

Corollary 30. Let $\left(h_{\mathbf{b}}^{\prime}\right)$ be the result of clearing a column in the $x_{j}$-direction on face $i$, as in the lemma, of a given $q$-tuple $\left(h_{\mathbf{b}}\right)$. Then

- $h_{\mathbf{b}}^{\prime}=h_{\mathbf{b}}$ for all $\mathbf{x}^{\mathbf{b}}$ on faces $1,2, \ldots, i-1$, and

- $h_{\mathbf{b}}^{\prime}=h_{\mathbf{b}}$ for all $\mathbf{x}^{\mathbf{b}}$ in any column in the $x_{v}$-direction for $2 \leq v \leq j$, other than the cleared column.

Proof. The lemma ensures that $h_{\mathbf{b}}=h_{\mathbf{b}}^{\prime}$ for all monomials $\mathbf{x}^{\mathbf{b}}$ on faces $1,2, \ldots, j-1$ except for those in the cleared column and its lower bound $m$, if the latter is on face $i$. It suffices to show that this set of monomials includes the monomials named in each bulleted statement. Since $i<j$, we know that the faces $i-1$ and lower are included among the faces $j-1$ and lower. Moreover, an "excepted" monomial either belongs to the cleared column, and is therefore on face $i$, or is equal to $m$, 
which is either on face $i$ or a face $j$ or higher, by Lemma 28. This shows that there are no "exceptions" among the monomials on faces $i-1$ or lower, which establishes the first bulleted statement. Now suppose that $\mathbf{x}^{\mathbf{b}}$ is a monomial in a column in the $x_{v}$-direction for $2 \leq v \leq j$, other than the cleared column. It follows by definition that $\mathbf{x}^{\mathbf{b}}$ lies on a face $\leq v-1 \leq j-1$. Since $\mathbf{x}^{\mathbf{b}}$ does not belong to the cleared column, by hypothesis, the only way that it can be "excepted" is for $\mathbf{x}^{\mathbf{b}}=m$ to belong to face $i$, in which case, by Lemma $28, \mathbf{x}^{\mathbf{b}}$ is either extreme or a member of a column in the $x_{j^{\prime}}$-direction for $j^{\prime}>j$, both of which are contradictions. This establishes the second bulleted statement and completes the proof.

We will call two distinct columns of $\mathfrak{B}(\beta)$ that lie in the same direction parallel columns.

Remark 31. The preceding corollary shows that if $\left(h_{\mathbf{b}}^{\prime}\right)$ is the result of clearing a column of a tuple $\left(h_{\mathbf{b}}\right)$ on face $i$ in the $x_{j}$-direction, then parallel columns are unaffected, that is, $h_{\mathbf{b}}^{\prime}=h_{\mathbf{b}}$ for all $\mathbf{x}^{\mathbf{b}}$ in any column parallel to the cleared column. In particular, this means that if we then clear any column of $\left(h_{\mathbf{b}}^{\prime}\right)$ parallel to the first cleared column, to obtain a third tuple $\left(h_{\mathbf{b}}^{\prime \prime}\right)$, then the components of $\left(h_{\mathbf{b}}^{\prime \prime}\right)$ corresponding to the first cleared column are still all equal to 0 (that is, still cleared). Furthermore, if the parallel column is on face $i$ or higher, then $h_{\mathbf{b}}=h_{\mathbf{b}}^{\prime}=h_{\mathbf{b}}^{\prime \prime}$ for all $\mathbf{x}^{\mathbf{b}}$ on faces $i-1$ or lower, by the first statement of the corollary. Finally, for $j^{\prime} \neq j$, (in particular, for $\left.1 \leq j^{\prime} \leq i\right)$, the differences

$$
h_{\mathbf{b}}^{\prime \prime}-h_{\mathbf{b}}=\left(h_{\mathbf{b}}^{\prime \prime}-h_{\mathbf{b}}^{\prime}\right)+\left(h_{\mathbf{b}}^{\prime}-h_{\mathbf{b}}\right)
$$

have $x_{j^{\prime}}$-degree at most equal to the maximum $x_{j^{\prime}}$-degree among the components $h_{\mathbf{b}_{k}}$ corresponding to monomials $\mathbf{x}^{\mathbf{b}_{k}}$ in the cleared columns. (This follows easily from the last statement of Lemma 29, together with the fact that $h_{\mathbf{b}}^{\prime}=h_{\mathbf{b}}$ for all $\mathbf{x}^{\mathbf{b}}$ in the second column to be cleared, since clearing the first column leaves parallel columns unaffected.) These observations enable us to clear parallel columns in succession, a technique that we exploit in the following lemma.

Lemma 32. Let $\left(h_{\mathbf{b}}\right)_{\mathbf{b} \in \mathfrak{B}(\beta)}$ be an arbitrary $q$-tuple of elements of $B[\mathbf{x}]$, and let $1 \leq i<n$. Then the coset $\left(h_{\mathbf{b}}\right)+\mathscr{S}$ contains a $q$-tuple $\left(h_{\mathbf{b}}^{*}\right)$ such that $h_{\mathbf{b}}^{*}=0$ for all non-extreme $\mathbf{x}^{\mathbf{b}}$ on faces $i$ and higher, and $h_{\mathbf{b}}^{*}=h_{\mathbf{b}}$ for all $\mathbf{x}^{\mathbf{b}}$ on faces lower than $i$. Furthermore, for $1 \leq j^{\prime} \leq i$, the differences $h_{\mathbf{b}}^{*}-h_{\mathbf{b}}$ have $x_{j^{\prime}}$-degree at most equal to the maximum $x_{j^{\prime}}$-degree among the components $h_{\mathbf{b}}$ corresponding to the non-extreme monomials $\mathbf{x}^{\mathbf{b}}$ on faces $i$ and higher - that is, the components $h_{\mathbf{b}}$ that are cleared.

Proof. We begin by clearing all the columns on face $i$ that are in the $(i+1)$ direction, one at a time. In light of Remark 31 , the result is a tuple $\left(h_{\mathbf{b}}^{1}\right) \in\left(h_{\mathbf{b}}\right)+\mathscr{Y}$ such that: $h_{\mathbf{b}}^{1}=0$ for all non-extreme $\mathbf{x}^{\mathbf{b}}$ on the $i$-th face that lie in a column in the $x_{i+1}$-direction; $h_{\mathbf{b}}^{1}=h_{\mathbf{b}}$ for all $\mathbf{x}^{\mathbf{b}}$ on faces lower than $i$; and the differences $h_{\mathbf{b}}^{1}-h_{\mathbf{b}}$ 
have $x_{j^{\prime}}$-degree at most equal to the maximum $x_{j^{\prime}}$-degree among the components of $\left(h_{\mathbf{b}}\right)$ that were cleared in the operation. If $i=n-1$, we are done. If $i<n-1$, we proceed by induction, and assume that we have constructed a tuple $\left(h_{\mathbf{b}}^{k}\right) \in\left(h_{\mathbf{b}}\right)+\mathscr{S}$ such that: $k<n-i ; h_{\mathbf{b}}^{k}=0$ for all non-extreme $\mathbf{x}^{\mathbf{b}}$ on faces $i, i+1, \ldots, i+k-1$ that lie in a column in the $x_{j}$-direction for $i+1 \leq j \leq i+k ; h_{\mathbf{b}}^{k}=h_{\mathbf{b}}$ for all $\mathbf{x}^{\mathbf{b}}$ on faces lower than $i$; and the differences $h_{\mathbf{b}}^{k}-h_{\mathbf{b}}$ have $x_{j^{\prime}}$-degree at most equal to the maximum $x_{j^{\prime}}$-degree among the components of $\left(h_{\mathbf{b}}\right)$ that have been cleared so far. We then clear all the columns of $\left(h_{\mathbf{b}}^{k}\right)$ in the $x_{i+k+1}$-direction on faces $i, i+1, \ldots, i+k$, to obtain a $q$-tuple $\left(h_{\mathbf{b}}^{k+1}\right) \in\left(h_{\mathbf{b}}\right)+\mathscr{Y}$. Corollary 30 and Remark 31 ensure that clearing these columns will leave unchanged all positions associated to monomials in columns that have already been cleared, and on faces lower than $i$. They also ensure that the differences $h_{\mathbf{b}}^{k+1}-h_{\mathbf{b}}^{k}$ have $x_{j^{\prime}}$-degree at most equal to the maximum $x_{j^{\prime}}$-degree among the components $h_{\mathbf{b}_{l}}^{k}$ corresponding to monomials $\mathbf{x}^{\mathbf{b}_{l}}$ belonging to the columns in the $x_{i+k+1}$-direction that we just cleared. The induction hypothesis on the $x_{j^{\prime}}$-degree of the differences $h_{\mathbf{b}}^{k}-h_{\mathbf{b}}$ holds in particular for the differences $h_{\mathbf{b}_{l}}^{k}-h_{\mathbf{b}_{l}}$, whence we obtain that the $x_{j^{\prime}}$-degree of the components

$$
h_{\mathbf{b}_{l}}^{k}=\left(h_{\mathbf{b}_{l}}^{k}-h_{\mathbf{b}_{l}}\right)+h_{\mathbf{b}_{l}}
$$

is at most equal to the maximum $x_{j^{\prime}}$-degree among the components of $\left(h_{\mathbf{b}}\right)$ corresponding to all of the cleared positions including those on the parallel columns in the $x_{i+k+1}$-direction. It now follows easily that the same is true for the differences

$$
h_{\mathbf{b}}^{k+1}-h_{\mathbf{b}}=\left(h_{\mathbf{b}}^{k+1}-h_{\mathbf{b}}^{k}\right)-\left(h_{\mathbf{b}}^{k}-h_{\mathbf{b}}\right) .
$$

The desired tuple is therefore obtained after the $(n-i)$-th iteration, which clears the columns in the $x_{n}$-direction on faces $i, i+1, \ldots, n-1$; that is, $\left(h_{\mathbf{b}}^{*}\right)=\left(h_{\mathbf{b}}^{n-i}\right)$.

We will call the process described in the preceding lemma (for producing $\left(h_{\mathbf{b}}^{*}\right)$ from $\left.\left(h_{\mathbf{b}}\right)\right)$ clearing all columns on faces $i$ or higher.

6.6. A reduced normal form for q-tuples. We can now establish the last technical result needed for the proof of Theorem 22. It is convenient to define a $q$-tuple $\left(h_{\mathbf{b}}\right)$ to be $i$-reduced provided that

- when $\mathbf{x}^{\mathbf{b}}$ is extreme and on the $j$-th face, then the tuple entry $h_{\mathbf{b}}$ only involves the variables $x_{l}, x_{l+1}, \ldots, x_{n}$, for $l=\min (i, j)$, and

- when $\mathbf{x}^{\mathbf{b}}$ is non-extreme, then the tuple entry $h_{\mathbf{b}}$ is 0 .

It is then clear that $\left(h_{\mathbf{b}}\right)$ is reduced (21) if and only if $\left(h_{\mathbf{b}}\right)$ is $n$-reduced.

Lemma 33. Let $\left(h_{\mathbf{b}}\right)_{\mathbf{b} \in \mathfrak{B}(\beta)}$ be an arbitrary $q$-tuple of elements of $B[\mathbf{x}]$. Then we can express $\left(h_{\mathbf{b}}\right)$ in the form

$$
\left(h_{\mathbf{b}}\right)=(\text { element of } \mathscr{S})+(\text { reduced tuple }) .
$$


In other words, every $\mathscr{S}$-coset contains at least one reduced representative.

Proof. We begin by clearing all columns on faces 1 or higher, to obtain a tuple $\left(h_{\mathbf{b}}^{c}\right) \in\left(h_{\mathbf{b}}\right)+\mathscr{Y}$ that is zero at all non-extreme positions. Let $t$ be the maximum $x_{1^{-}}$ degree among the components $h_{\mathbf{b}}^{c}$ corresponding to extreme monomials $x_{\mathbf{b}}$ on faces 2 or higher. If $t>0$, apply the operation of lowering the $x_{1}$-degree at each $\mathbf{b}_{1}$ such that the $x_{1}$-degree of $h_{\mathbf{b}_{1}}^{c}$ is $t$. Recalling Lemma 27, we see that the result is a tuple $\left(h_{\mathbf{b}}^{d}\right) \in\left(h_{\mathbf{b}}\right)+\mathscr{Y}$ such that the $x_{1}$-degree of every component $h_{\mathbf{b}}^{d}$, with the possible exception of the component corresponding to the unique extreme monomial $x_{1}^{w_{1}}$ on face 1 , is at most $t-1$. We again clear all columns on faces 1 and higher to obtain a tuple $\left(h_{\mathbf{b}}^{c c}\right)$ such that $h_{\mathbf{b}}^{c c}=0$ at all non-extreme positions and the maximum $x_{1}$-degree among the extreme positions on faces 2 and higher is still at most $t-1$, by Lemma 32. It is now clear that we can repeat the operations of lowering the maximal $x_{1}$-degree and column clearing as often as necessary until we construct a tuple $\left(h_{\mathbf{b}}^{1}\right) \in\left(h_{\mathbf{b}}\right)+\mathscr{S}$ that is zero in all non-extreme positions and has $x_{1}$-degree zero at every extreme position on faces 2 and higher - that is, $\left(h_{\mathbf{b}}^{1}\right)$ is 2-reduced.

If $n=2$, we are done: the tuple $\left(h_{\mathbf{b}}^{1}\right)$ is reduced. If $n>2$, we proceed by induction. Suppose that we have constructed a $q$-tuple $\left(h_{\mathbf{b}}^{k}\right) \in\left(h_{\mathbf{b}}\right)+\mathscr{Y}$ such that $\left(h_{\mathbf{b}}^{k}\right)$ is $(k+1)$-reduced for $k+1<n$. We then carry out on $\left(h_{\mathbf{b}}^{k}\right)$ the alternation of reduction of $x_{k+1}$-degree at extreme positions on faces $k+2$ and higher having maximum $x_{k+1}$-degree, followed by the clearing of all columns on faces $k+1$ and higher, until the maximum $x_{k+1}$-degree on faces $k+2$ and higher has dropped to zero. The result is a tuple

$$
\left(h_{\mathbf{b}}^{k+1}\right) \in\left(h_{\mathbf{b}}\right)+\mathscr{Y},
$$

which we claim is $(k+2)$-reduced. Assuming the claim, we see that the desired reduced tuple is produced after $n-1$ iterations, since $\left(h_{\mathbf{b}}^{n-1}\right)$ is $n$-reduced. It remains to prove the claim, which we proceed to do.

We first remark that $h_{\mathbf{b}}^{k}=h_{\mathbf{b}}^{k+1}$ for all $\mathbf{x}^{\mathbf{b}}$ on faces $k$ and lower, because both the degree reduction and the column clearing steps leave these faces unaffected: Indeed, we lower $x_{k+1}$-degree on faces $k+2$ and higher, and such operations (can be chosen to) preserve faces $k$ and lower by Lemma 27, and we clear all columns on faces $k+1$ and higher, and this operation preserves faces $k$ and lower by Lemma 32. In particular, $h_{\mathbf{b}}^{k+1}=0$ for all non-extreme monomials $\mathbf{x}^{\mathbf{b}}$ on faces $k$ and lower, and $h_{\mathbf{b}}^{k+1}$ involves only the variables $x_{l}, x_{l+1}, \ldots, x_{n}$ for extreme $\mathbf{x}^{\mathbf{b}}$ on faces $1 \leq l \leq k$. By construction, $h_{\mathbf{b}}^{k+1}=0$ at all non-extreme positions on faces $k+1, \ldots, n-1$, and the extreme positions on faces $k+2$ and higher do not involve the variable $x_{k+1}$. To show that $\left(h_{\mathbf{b}}^{k+1}\right)$ is $(k+2)$-reduced, it now suffices to show that the extreme positions on faces $k+1$ and higher in this tuple cannot involve $x_{1}, \ldots, x_{k}$. However, the induction hypothesis shows that this condition is true for $\left(h_{\mathbf{b}}^{k}\right)$, and neither the degree reduction nor the column clearing steps can introduce 
these variables. For degree reduction, this follows from equation (25): We are lowering $x_{k+1}$-degree at $\mathbf{b}_{1}$ on face $k+2$ and higher, and we can choose the basic pseudosyzygy $P\left(\mathbf{b}_{1}, \mathbf{b}_{2}\right)$ so that its components $p_{\mathbf{b}}$ are either constants or involve a variable, either $x_{k+1}$ or $x_{l}$, where $l>k+1$ is the face that $\mathbf{x}^{\mathbf{b}_{1}}$ lies on (see the proof of Lemma 27). Since we are assuming that $h_{\mathbf{b}_{1}}$ and (hence) $\gamma$ do not involve the variables $x_{1}, \ldots, x_{k}$, we see from (25) that the tuple components that result from the operation cannot involve $x_{1}, \ldots, x_{k}$ if the corresponding original components do not. For column clearing, recall that we are clearing all the columns on faces $k+1$ and higher, assuming that the components of the original tuple corresponding to monomials on faces $k+1$ and higher do not involve the variables $x_{1}, \ldots, x_{k}$; in particular, the maximal degree of any of these variables appearing among the components that are to be cleared is 0. It now follows from Lemma 32 that the resulting components can differ from the original components by at most degree 0 in $x_{1}, \ldots, x_{k}$, showing that if any original component lacks these variables, then so does the corresponding resulting component. This completes the proof that $\left(h_{\mathbf{b}}^{k+1}\right)$ is $(k+2)$-reduced, and we are done.

Remark 34. The base case of the inductive argument just presented was used (in slightly different form) to prove [Huibregtse 2002, Lemma 4.3.3, p. 116].

\section{Construction of $\left(U_{\beta}, Z_{\beta}\right)$}

Let $\mathrm{k}$ be any commutative and unitary ring, and $\beta$ a basis set of $d$ monomials in the variables $x_{1}, x_{2}, \ldots, x_{n}$. In this section of the paper we construct an affine scheme $U_{\beta}$ of finite type over $\operatorname{Spec}(\mathrm{k})$, and a family of subschemes $Z_{\beta} \subseteq U_{\beta} \times{ }_{\mathrm{k}} A_{\mathrm{k}}^{n}$ such that the direct image $2_{\beta}$ of the structure sheaf of $Z_{\beta}$ on $U_{\beta}$ is free with basis $\beta$, and the pair $\left(U_{\beta}, Z_{\beta}\right)$ represents the functor $F_{\mathrm{A}_{\mathrm{k}}^{n}}^{\beta}$ from (6). By Lemma 7, the pairs $\left(U_{\beta}, Z_{\beta}\right)$ can be glued to yield the multigraded Hilbert scheme of points $\left(\mathbf{H}_{\AA_{k}^{n}}^{d}, Z_{A_{k}^{n}}^{d}\right)$. We present several examples of the schemes $U_{\beta}$ in Section 8 .

7.1. Preliminary definitions and notation. We introduce the set of indeterminates

$$
\mathbf{C}=\left\{C_{\mathbf{j}}^{\mathbf{b}} \mid \mathbf{b} \in \mathfrak{B}(\beta), \mathbf{j} \in \beta\right\},
$$

and define the polynomials

$$
G_{\mathbf{b}}=\mathbf{x}^{\mathbf{b}}-\sum_{\mathbf{j} \in \beta} C_{\mathbf{j}}^{\mathbf{b}} \cdot \mathbf{x}^{\mathbf{j}}, \quad \mathbf{b} \in \mathfrak{B}(\beta),
$$

which are of the form (14). For each basic pseudosyzygy $P\left(\mathbf{b}_{1}, \mathbf{b}_{2}\right)$ of the $G_{\mathbf{b}}$, we write (recalling (15))

$$
P\left(\mathbf{b}_{1}, \mathbf{b}_{2}\right) \cdot\left(G_{\mathbf{b}}\right)=\sum_{\mathbf{j} \in \beta} \rho_{\mathbf{j}}^{\left(\mathbf{b}_{1}, \mathbf{b}_{2}\right)} \mathbf{x}^{\mathbf{j}}, \quad \rho_{\mathbf{j}}^{\left(\mathbf{b}_{1}, \mathbf{b}_{2}\right)} \in \mathrm{k}[\mathbf{C}] .
$$


For example, recall the discussion in Section 1.4 of the basis set $\beta=\left\{1, x_{1}\right\} \subseteq$ $\mathrm{k}\left[x_{1}, x_{2}\right]$. The boundary monomials are

$$
\mathbf{x}^{\mathbf{b}_{1}}=x_{2}, \quad \mathbf{x}^{\mathbf{b}_{2}}=x_{1} x_{2}, \quad \mathbf{x}^{\mathbf{b}_{3}}=x_{1}^{2},
$$

and the adjacent pairs (up to order) are

$$
\left(\mathbf{b}_{1}, \mathbf{b}_{2}\right) \text { and }\left(\mathbf{b}_{2}, \mathbf{b}_{3}\right) \text {. }
$$

We can re-express (4) and (5) in our current notation as

$$
\begin{aligned}
& \rho_{(0,0)}^{\left(\mathbf{b}_{1}, \mathbf{b}_{2}\right)}=-C_{(0,0)}^{(1,1)}+C_{(1,0)}^{(0,1)} C_{(0,0)}^{(2,0)}, \\
& \rho_{(1,0)}^{\left(\mathbf{b}_{1}, \mathbf{b}_{2}\right)}=C_{(0,0)}^{(0,1)}-C_{(1,0)}^{(1,1)}+C_{(1,0)}^{(0,1)} C_{(1,0)}^{(2,0)}, \\
& \rho_{(0,0)}^{\left(\mathbf{b}_{2}, \mathbf{b}_{3}\right)}=-C_{(0,0)}^{(0,1)} C_{(0,0)}^{(2,0)}+C_{(1,0)}^{(1,1)} C_{(0,0)}^{(2,0)}-C_{(0,0)}^{(1,1)} C_{(1,0)}^{(2,0)}, \\
& \rho_{(1,0)}^{\left(\mathbf{b}_{2}, \mathbf{b}_{3}\right)}=C_{(0,0)}^{(1,1)}-C_{(1,0)}^{(0,1)} C_{(0,0)}^{(2,0)} .
\end{aligned}
$$

Remark 35. By equating coefficients in equation (30), while recalling the form of the entries of $P\left(\mathbf{b}_{1}, \mathbf{b}_{2}\right)$ given in equations (18) and (19), one sees that the coefficients $\rho_{\mathbf{j}}^{\left(\mathbf{b}_{1}, \mathbf{b}_{2}\right)}$ can involve only linear and quadratic terms in the indeterminates $C_{\mathbf{j}}^{\mathbf{b}}$ (possibly reducing to zero, as in Section 8.2). Moreover, the only way that the indeterminate $C_{\mathbf{j}^{\prime}}^{\mathbf{b}^{\prime}}$ can occur linearly in $\rho_{\mathbf{j}}^{\left(\mathbf{b}_{1}, \mathbf{b}_{2}\right)}$ is as the coefficient of one of the two following products on the left-hand side of (30), if present.

$$
\begin{array}{rr}
-z_{1} \cdot\left(-C_{\mathbf{j}^{\prime}}^{\mathbf{b}_{1}} \mathbf{x}^{\mathbf{j}^{\prime}}\right), & \text { where } z_{1} \cdot \mathbf{x}^{\mathbf{j}^{\prime}}=\mathbf{x}^{\mathbf{j}} ; \\
z_{2} \cdot\left(-C_{\mathbf{j}^{\prime}}^{\mathbf{b}_{2}} \mathbf{x}^{\mathbf{j}^{\prime}}\right), & \text { where } z_{2} \cdot \mathbf{x}^{\mathbf{j}^{\prime}}=\mathbf{x}^{\mathbf{j}} .
\end{array}
$$

In other words, in order for $C_{\mathbf{j}^{\prime}}^{\mathbf{b}^{\prime}}$ to occur linearly in $\rho_{\mathbf{j}}^{\left(\mathbf{b}_{1}, \mathbf{b}_{2}\right)}$, it is necessary that $\mathbf{b}^{\prime}=\mathbf{b}_{i}$ for $i=1$ or 2 , and that $\mathbf{x}^{\mathbf{j}} / z_{i}=\mathbf{x}^{\mathbf{j}^{\prime}}$ (in particular, there can be at most two linear terms in any of the polynomials $\left.\rho_{\mathbf{j}}^{\left(\mathbf{b}_{1}, \mathbf{b}_{2}\right)}\right)$. These observations will be useful in Section 8.4 and Section 8.5.

7.2. The coordinate ring $\boldsymbol{R}_{\boldsymbol{\beta}}$ of $\boldsymbol{U}_{\boldsymbol{\beta}}$. Let $\mathfrak{R} \subseteq \mathrm{k}[\mathbf{C}]$ denote the ideal generated by the polynomials $\rho_{\mathbf{j}}^{\left(\mathbf{b}_{1}, \mathbf{b}_{2}\right)}$, and

$$
R=R_{\beta}=\mathrm{k}[\mathbf{C}] / \Re
$$

the quotient ring. We write $\hat{\delta}: \mathrm{k}[\mathbf{C}][\mathbf{x}] \rightarrow R[\mathbf{x}]$ for the natural map induced by the quotient map $\delta: \mathrm{k}[\mathbf{C}] \rightarrow R$, and let $\widehat{G}_{\mathbf{b}}=\hat{\delta}\left(G_{\mathbf{b}}\right)$ for $\mathbf{b} \in \mathfrak{B}(\beta)$. We denote by $\widehat{J} \subseteq R[\mathbf{x}]$ the ideal generated by the $\widehat{G}_{\mathbf{b}}$.

Lemma 36. The quotient $R[\mathbf{x}] / \widehat{J}$ is $R$-free with basis $\beta$. 
Proof. Lemma 21 shows that $\hat{\delta}\left(P\left(\mathbf{b}_{1}, \mathbf{b}_{2}\right)\right)=\widehat{P}\left(\mathbf{b}_{1}, \mathbf{b}_{2}\right)$ is the basic pseudosyzygy of the $\widehat{G}_{\mathbf{b}}$ corresponding to the adjacent pair $\left(\mathbf{b}_{1}, \mathbf{b}_{2}\right)$. Moreover, using (30), we see that

$\widehat{P}\left(\mathbf{b}_{1}, \mathbf{b}_{2}\right) \cdot\left(\widehat{G}_{\mathbf{b}}\right)=\hat{\delta}\left(P\left(\mathbf{b}_{1}, \mathbf{b}_{2}\right) \cdot\left(G_{\mathbf{b}}\right)\right)=\hat{\delta}\left(\sum_{\mathbf{j} \in \beta} \rho_{\mathbf{j}}^{\left(\mathbf{b}_{1}, \mathbf{b}_{2}\right)} \mathbf{x}^{\mathbf{j}}\right)=\sum_{\mathbf{j} \in \beta} \delta\left(\rho_{\mathbf{j}}^{\left(\mathbf{b}_{1}, \mathbf{b}_{2}\right)}\right) \mathbf{x}^{\mathbf{j}}=0$,

since the coefficients $\rho_{\mathbf{j}}^{\left(\mathbf{b}_{1}, \mathbf{b}_{2}\right)}$ all map to 0 under $\delta$. In other words, the basic pseudosyzygies of the $\widehat{G}_{\mathbf{b}}$ are syzygies; therefore, Theorem 22 applies and yields the result.

\subsection{The universal pair $\left(U_{\beta}, Z_{\beta}\right)$.}

Theorem 37. Let $\beta \subseteq \mathrm{k}[x]$ be a basis set of $d$ monomials. Then there exists a pair $\left(U_{\beta}, Z_{\beta}\right)$ consisting of an affine scheme $U_{\beta}$ of finite type over $\mathrm{k}$, and a closed subscheme $Z_{\beta} \subseteq U_{\beta} \times_{\mathrm{k}} \mathbb{A}_{\mathrm{k}}^{n}$, that represents the functor $F_{\mathrm{A}_{\mathrm{k}}^{n}}^{\beta}$ from (6).

Proof. We define

$$
\begin{aligned}
U_{\beta} & =\operatorname{Spec}(R), \\
Z_{\beta} & =\operatorname{Spec}(R[\mathbf{x}] / \widehat{J}) \subseteq U_{\beta} \times{ }_{\mathrm{k}} A_{A}^{n}=\operatorname{Spec}(R[\mathbf{x}]) .
\end{aligned}
$$

It is clear that $U_{\beta}$ is an affine scheme of finite type over $\mathrm{k}$, and $Z_{\beta}$ is a closed subscheme of $U_{\beta} \times_{k} \mathbb{A}_{k}^{n}$; furthermore, by Lemma 36 , the direct image $2_{\beta}$ of the structure sheaf of $Z_{\beta}$ on $U_{\beta}$ is free with basis $\beta$.

Most importantly, the pair $\left(U_{\beta}, Z_{\beta}\right)$ represents the functor $F_{\mathrm{A}_{k}^{n}}^{\beta}$. Indeed, let $T$ be a k-scheme. We must show that a map $f: T \rightarrow U_{\beta}$ corresponds uniquely to a closed subscheme $Z_{f} \subseteq T \times \times_{k} \mathbb{A}_{A}^{n}$ whose direct image $2_{f}$ on $T$ is free with basis $\beta$, the correspondence being defined by $f \mapsto f^{*}\left(Z_{\beta}\right)$.

Given $f: T \rightarrow U_{\beta}$, it is clear that $Z_{f}=f^{*}\left(Z_{\beta}\right)$ has the desired property, since $Z_{\beta}$ does. So, let $Z \subseteq T \times{ }_{\mathrm{k}} \mathbb{A}_{A}^{n}$ be such that its direct image 2 on $T$ is free with basis $\beta$. In particular, this means that the module of global sections of 2 is a quotient of $\Gamma\left(\mathrm{O}_{T}\right)[\mathbf{x}]$ that is $\Gamma\left(\mathrm{O}_{T}\right)$-free with basis $\beta$; therefore, by Proposition 12, there are unique global functions $t_{\mathbf{j}}^{\mathbf{b}}$ on $T$ such that

$$
\Gamma(2)=\Gamma\left(\mathcal{O}_{T}\right)[\mathbf{x}] /\left(F_{\mathbf{b}}\right),
$$

where

$$
F_{\mathbf{b}}=\mathbf{x}^{\mathbf{b}}-\sum_{\mathbf{j} \in \beta} t_{\mathbf{j}}^{\mathbf{b}} \mathbf{x}^{\mathbf{j}}, \quad \mathbf{b} \in \mathfrak{B}(\beta) .
$$

Furthermore, the basic pseudosyzygies of the $F_{\mathbf{b}}$ are all syzygies, by Theorem 22 .

Let $z: \mathrm{k}[\mathbf{C}] \rightarrow \Gamma\left(\mathbb{O}_{T}\right)$ be the ring homomorphism defined by $C_{\mathbf{j}}^{\mathbf{b}} \mapsto t_{\mathbf{j}}^{\mathbf{b}}$, and let $\hat{z}$ denote the induced map $\mathrm{k}[\mathbf{C}][\mathbf{x}] \rightarrow \Gamma\left(\mathbb{O}_{T}\right)[\mathbf{x}]$. By Lemma 21, the basic pseudosyzygies of the $F_{\mathbf{b}}=\hat{z}\left(G_{\mathbf{b}}\right)$ are of the form $\hat{z}\left(P\left(\mathbf{b}_{1}, \mathbf{b}_{2}\right)\right)$ (applied componentwise), 
where the $P\left(\mathbf{b}_{1}, \mathbf{b}_{2}\right)$ are the basic pseudosyzygies of the $G_{\mathbf{b}}$. Accordingly, since the basic pseudosyzygies of the $F_{\mathbf{b}}$ are syzygies, we see (again using (30)) that

$$
\begin{aligned}
0=\hat{z}\left(P\left(\mathbf{b}_{1}, \mathbf{b}_{2}\right)\right) \cdot\left(F_{\mathbf{b}}\right) & =\hat{z}\left(P\left(\mathbf{b}_{1}, \mathbf{b}_{2}\right)\right) \cdot\left(\hat{z}\left(G_{\mathbf{b}}\right)\right)=\hat{z}\left(P\left(\mathbf{b}_{1}, \mathbf{b}_{2}\right) \cdot\left(G_{\mathbf{b}}\right)\right) \\
& =\hat{z}\left(\sum_{\mathbf{j} \in \beta} \rho_{\mathbf{j}}^{\left(\mathbf{b}_{1}, \mathbf{b}_{2}\right)} \mathbf{x}^{\mathbf{j}}\right)=\sum_{\mathbf{j} \in \beta} z\left(\rho_{\mathbf{j}}^{\left(\mathbf{b}_{1}, \mathbf{b}_{2}\right)}\right) \mathbf{x}^{\mathbf{j}} \in \Gamma\left(\mathcal{O}_{T}\right)[\mathbf{x}],
\end{aligned}
$$

which implies that $z\left(\rho_{\mathbf{j}}^{\left(\mathbf{b}_{1}, \mathbf{b}_{2}\right)}\right)=0$ for all adjacent pairs $\left(\mathbf{b}_{1}, \mathbf{b}_{2}\right)$ and all $\mathbf{x}^{\mathbf{j}} \in \beta$. It follows that $z$ induces a map $R \rightarrow \Gamma\left(\mathcal{O}_{T}\right)$ which in turn yields a map $f: T \rightarrow U_{\beta}$ that one sees is the unique possible map such that $f^{*}\left(Z_{\beta}\right)=Z=Z_{f}$.

Remark 38. Since $P\left(\mathbf{b}_{2}, \mathbf{b}_{1}\right)=-P\left(\mathbf{b}_{1}, \mathbf{b}_{2}\right)$, as noted in Remark 18 , we have that $\rho_{\mathbf{j}}^{\left(\mathbf{b}_{2}, \mathbf{b}_{1}\right)}=-\rho_{\mathbf{j}}^{\left(\mathbf{b}_{1}, \mathbf{b}_{2}\right)}$. Therefore, in computing generators of the ideal $\mathfrak{R}$, we only need to use one of the members of each subset

$$
\left\{\left(\mathbf{b}_{1}, \mathbf{b}_{2}\right),\left(\mathbf{b}_{2}, \mathbf{b}_{1}\right)\right\}
$$

of adjacent pairs. Furthermore, it suffices to consider only the adjacent pairs that are needed for the degree lowering and column clearing operations used to prove Lemma 33.

\section{Examples}

To conclude this paper, we present a series of examples of the schemes $U_{\beta}$. Recall that we are working over a commutative and unitary base ring $k$, unless otherwise stated.

8.1. Example 1. Let $n=1$, let $d$ be any positive integer, and let

$$
\beta=\left\{1, x_{1}, x_{1}^{2}, \ldots, x_{1}^{d-1}\right\} .
$$

In one variable, there is only one basis set $\beta$ for each colength. The set of boundary monomials is the singleton set $\mathfrak{B}(\beta)=\left\{x_{1}^{d}\right\}$; therefore, there are no adjacent pairs of boundary monomials, and hence no basic pseudosyzygies. Consequently, there are no relations $\rho_{\mathbf{j}}^{\left(\mathbf{b}_{1}, \mathbf{b}_{2}\right)}$ among the coefficients of the (single, in this case) polynomial (29)

$$
G_{(d)}=x_{1}^{d}-C_{(d-1)}^{(d)} x_{1}^{d-1}-\cdots-C_{(1)}^{(d)} x_{1}^{1}-C_{(0)}^{(d)} ;
$$

whence, the coefficient set $\mathbf{C}=\left\{C_{(d-1)}^{(d)}, \ldots, C_{(0)}^{(d)}\right\}$, and the ideal $\mathfrak{R}=0$. It follows that (recall (31))

$$
\begin{aligned}
\mathbf{H}_{\mathrm{A}_{\mathrm{k}}^{1}}^{d} & =U_{\beta}=\operatorname{Spec}(R)=\operatorname{Spec}(\mathrm{k}[\mathbf{C}] / \mathfrak{R})=\operatorname{Spec}(\mathrm{k}[\mathbf{C}]), \\
Z_{\mathrm{A}_{\mathrm{k}}^{1}}^{d} & =Z_{\beta}=\mathrm{k}[\mathbf{C}]\left[x_{1}\right] /\left(G_{(d)}\right) .
\end{aligned}
$$


Now let $t_{1}, t_{2}, \ldots, t_{d}$ be indeterminates, let $T=\operatorname{Spec}\left(\mathrm{k}\left[t_{1}, \ldots, t_{d}\right]\right)$, let $\mathbb{A}_{\mathrm{k}}^{1}=$ $\operatorname{Spec}\left(\mathrm{k}\left[x_{1}\right]\right)$, and let

$$
\Delta=\prod_{j=1}^{d}\left(x_{1}-t_{j}\right)=x_{1}^{d}-\sum_{i=1}^{d}(-1)^{i-1} s_{i} x_{i}^{d-i} \in \mathrm{k}\left[t_{1}, \ldots, t_{d}\right]\left[x_{1}\right],
$$

where the $s_{i}$ are the elementary symmetric polynomials in the $t_{j}$. It is clear that the quotient $\mathrm{k}\left[t_{1}, \ldots, t_{d}\right]\left[x_{1}\right] /(\Delta)$ is $\mathrm{k}\left[t_{1}, \ldots, t_{d}\right]$-free with basis $\beta$; therefore, the universal property of $U_{\beta}$ yields a map $T \rightarrow U_{\beta}$ with comorphism $\mathrm{k}[\mathbf{C}] \rightarrow \mathrm{k}\left[t_{1}, \ldots, t_{d}\right]$ given by $C_{(d-i)}^{(d)} \mapsto(-1)^{i-1} s_{i}$, which is evidently an isomorphism onto the ring of invariants

$$
\mathrm{k}\left[s_{1}, \ldots, s_{d}\right]=\mathrm{k}\left[t_{1}, \ldots, t_{d}\right]^{S_{d}} \subseteq \mathrm{k}\left[t_{1}, \ldots, t_{d}\right]
$$

under the natural action of the symmetric group on $d$ letters. In this way we recover the fact that the Hilbert scheme of $d$ points of the affine line is isomorphic to the $d$ fold symmetric product of the affine line, with the universal subscheme cut out by the polynomial $\Delta$. Skjelnes [2002] gives an alternative demonstration of this fact, and, more generally, constructs the Hilbert schemes of $d$ points of localizations of the affine line.

8.2. Example 2. Let $n=2$ (for simplicity; any positive integer would work), let $d=1$, and let $\beta=\{1\}$.

If the colength is 1 , there is again only one possible basis set. The set of boundary monomials is $\mathfrak{B}(\beta)=\left\{x_{1}, x_{2}\right\}$. The polynomials (29) are then

$$
G_{(1,0)}=x_{1}-C_{(0,0)}^{(1,0)}, \quad G_{(0,1)}=x_{2}-C_{(0,0)}^{(0,1)} .
$$

There is only one adjacent pair of boundary monomials (up to order), namely $\left(x_{1}, x_{2}\right)$, or $\left(\mathbf{b}_{1}, \mathbf{b}_{2}\right)=((1,0),(0,1))$ in exponent-list notation. The associated basic pseudosyzygy $P\left(\mathbf{b}_{1}, \mathbf{b}_{2}\right)$ is easily computed to be

$$
P\left(\mathbf{b}_{1}, \mathbf{b}_{2}\right)=\left(-x_{2}+C_{(0,0)}^{(0,1)}, x_{1}-C_{(0,0)}^{(1,0)}\right) .
$$

The $\rho_{\mathbf{j}}^{\left(\mathbf{b}_{1}, \mathbf{b}_{2}\right)}$ are then the coefficients of the basis monomials in the expression

$$
\begin{aligned}
P\left(\mathbf{b}_{1}, \mathbf{b}_{2}\right) \cdot\left(G_{(1,0)}, G_{(0,1)}\right) & =\left(-x_{2}+C_{(0,0)}^{(0,1)}, x_{1}-C_{(0,0)}^{(1,0)}\right) \cdot\left(x_{1}-C_{(0,0)}^{(1,0)}, x_{2}-C_{(0,0)}^{(0,1)}\right) \\
& =0 \cdot 1,
\end{aligned}
$$

of which there is only one, namely

$$
\rho_{(0,0)}^{((1,0),(0,1))}=0 .
$$

Therefore, the set of coefficients $\mathbf{C}=\left\{C_{(0,0)}^{(1,0)}, C_{(0,0)}^{(0,1)}\right\}$, and the ideal $\Re=0$. It follows that 


$$
\begin{aligned}
U_{\beta} & =\operatorname{Spec}(R)=\operatorname{Spec}(\mathrm{k}[\mathbf{C}] / \Re)=\operatorname{Spec}(\mathrm{k}[\mathbf{C}]), \\
Z_{\beta} & =\mathrm{k}[\mathbf{C}]\left[x_{1}, x_{2}\right] /\left(x_{1}-C_{(0,0)}^{(1,0)}, x_{2}-C_{(0,0)}^{(0,1)}\right) .
\end{aligned}
$$

This confirms the general fact that Hilb $_{X}^{1}$ is isomorphic to $X$, with the universal subscheme $Z_{X}^{1} \subseteq X \times_{\mathrm{k}} X$ being the diagonal, when $X$ is the affine plane.

8.3. Example 3. Let $n=2$, let $d=3$, and let $\beta=\left\{1, x_{1}, x_{2}\right\}$.

The basis and boundary monomials are shown in the following diagram, with the latter in bold face.

$$
\begin{array}{ccc}
\mathbf{x}_{2}^{2} & & \\
x_{2} & \mathbf{x}_{1} \mathbf{x}_{2} & \\
1 & x_{1} & \mathbf{x}_{1}^{2}
\end{array}
$$

The set of polynomials (29) in this case contains the three members

$$
\begin{aligned}
& G_{(2,0)}=x_{1}^{2}-C_{(0,0)}^{(2,0)}-C_{(1,0)}^{(2,0)} x_{1}-C_{(0,1)}^{(2,0)} x_{2}, \\
& G_{(1,1)}=x_{1} x_{2}-C_{(0,0)}^{(1,1)}-C_{(1,0)}^{(1,1)} x_{1}-C_{(0,1)}^{(1,1)} x_{2}, \\
& G_{(0,2)}=x_{2}^{2}-C_{(0,0)}^{(0,2)}-C_{(1,0)}^{(0,2)} x_{1}-C_{(0,1)}^{(0,2)} x_{2} .
\end{aligned}
$$

Up to order, there are two adjacent pairs of boundary monomials, namely $\left(x_{1}^{2}, x_{1} x_{2}\right)$ and $\left(x_{1} x_{2}, x_{2}^{2}\right)$, or in exponent-list notation, $((2,0),(1,1))$ and $((1,1),(0,2))$. The associated basic pseudosyzygies are

$$
\begin{aligned}
& P((2,0),(1,1))=\left(-x_{2}+C_{(1,0)}^{(1,1)}, x_{1}-C_{(1,0)}^{(2,0)}+C_{(0,1)}^{(1,1)},-C_{(0,1)}^{(2,0)}\right) \\
& P((1,1),(0,2))=\left(C_{(1,0)}^{(0,2)},-x_{2}-C_{(1,0)}^{(1,1)}+C_{(0,1)}^{(0,2)}, x_{1}-C_{(0,1)}^{(1,1)}\right) .
\end{aligned}
$$

The two dot products $P\left(\mathbf{b}_{1}, \mathbf{b}_{2}\right) \cdot\left(G_{\mathbf{b}}\right)$ yield six relations $\rho_{\mathbf{j}}^{\left(\mathbf{b}_{1}, \mathbf{b}_{2}\right)}$ among the $C_{\mathbf{j}}^{\mathbf{b}}$ that we do not list here; see [Huibregtse 2002, Section 5.2, p. 125], where this example is discussed in detail (using slightly different notation). The relations imply that

$$
R=\mathrm{k}[\mathbf{C}] / \Re=\mathrm{k}\left[C_{(1,0)}^{(2,0)}, C_{(0,1)}^{(2,0)}, C_{(1,0)}^{(1,1)}, C_{(0,1)}^{(1,1)}, C_{(1,0)}^{(0,2)}, C_{(0,1)}^{(0,2)}\right]
$$

is a polynomial ring in six indeterminates, so that

$$
U_{\beta}=\operatorname{Spec}(R) \approx \mathbb{A}_{\mathrm{k}}^{6}
$$

This example is generalized in [Huibregtse 2002], where sufficient conditions for $U_{\beta}$ to be an affine space (in the case of $n=2$ variables) are established; in particular, these conditions hold for all basis sets in two variables of the form

$$
\beta=\{\text { monomials } m \mid \text { total degree of } m \leq s\}, \quad s=0,1,2, \ldots
$$


8.4. Example 4. Let $n=3$, let $d=4$, and let $\beta=\left\{1, x_{1}, x_{2}, x_{3}\right\}$.

In this case the boundary monomials are the six quadratic monomials in three variables:

$$
\mathfrak{B}(\beta)=\left\{x_{1}^{2}, x_{1} x_{2}, x_{1} x_{3}, x_{2}^{2}, x_{2} x_{3}, x_{3}^{2}\right\} .
$$

There are accordingly six polynomials $G_{\mathbf{b}}$ from (29), and twenty-four indeterminates $C_{\mathbf{j}}^{\mathbf{b}}$. An adjacent pair consists in this case of two distinct boundary monomials that share a variable. There are 9 such pairs in all, up to order; for example, here are the three in which $x_{1}$ is the shared variable, in exponent-list notation:

$$
((2,0,0),(1,1,0)), \quad((2,0,0),(1,0,1)), \quad((1,1,0),(1,0,1)) .
$$

Accordingly, there are $4 \cdot 9=36$ coefficients $\rho_{\mathbf{j}}^{\left(\mathbf{b}_{1}, \mathbf{b}_{2}\right)}$ that generate the ideal $\Re \subseteq$ $k[\mathbf{C}]$.

We remark that for any adjacent pair $\left(\mathbf{b}_{1}, \mathbf{b}_{2}\right)$, the values $z_{1}$ and $z_{2}$ used in constructing the basic pseudosyzygy $P\left(\mathbf{b}_{1}, \mathbf{b}_{2}\right)$ (see Section 5.3) are both variables. Recalling now the necessary condition for the indeterminate $C_{\mathbf{j}^{\prime}}^{\mathbf{b}^{\prime}}$ to appear linearly in $\rho_{\mathbf{j}}^{\left(\mathbf{b}_{1}, \mathbf{b}_{2}\right)}$ that we observed in Remark 35 , we find that the indeterminate $C_{\mathbf{j}^{\prime}}^{\mathbf{b}^{\prime}}$ can appear linearly in $\rho_{\mathbf{j}}^{\left(\mathbf{b}_{1}, \mathbf{b}_{2}\right)}$ only if $\mathbf{j}^{\prime}=(0,0,0)$. From this it follows that the $6 \cdot 3=18$ indeterminates

$$
C_{\mathbf{j}}^{\mathbf{b}} \quad \text { with } \mathbf{j} \neq(0,0,0),
$$

must be k-linearly independent modulo the square of the ideal generated by the set of indeterminates $\mathbf{C}$, from (28), in the coordinate ring $\mathrm{k}[\mathbf{C}] / \mathfrak{R}$ of $U_{\beta}$.

Now restrict to the case of an algebraically closed field $\mathrm{k}$. Let $p$ denote the "origin" of $U_{\beta} \subseteq \mathbf{H}_{\mathrm{A}_{\mathrm{k}}^{3}}^{4}$; that is, the k-point at which all the coordinate functions $C_{\mathbf{j}}^{\mathbf{b}}$ vanish. One sees easily that $p$ corresponds to the monomial ideal generated by the six boundary monomials (32). Let $\mathfrak{m} \subseteq \mathrm{k}[\mathbf{C}] / \mathfrak{R}$ denote the maximal ideal of $p$; it is clear that $\mathfrak{m}$ is generated by the set of indeterminates $\mathbf{C}$. In light of the last paragraph, we observe that the $\mathrm{k}$-vector space $\mathfrak{m} / \mathfrak{m}^{2}$ has dimension at least 18. If $p$ were a smooth point of $\mathbf{H}_{\mathrm{A}_{k}^{3}}^{d}$, then the 18 indeterminates (33) would be algebraically independent in the coordinate ring of $U_{\beta}$. However, this is not the case; for example,

$$
\begin{aligned}
\rho_{(1,0,0)}^{((1,1,0),(1,0,1))} & =-C_{(1,0,0)}^{(0,2,0)} C_{(0,1,0)}^{(1,0,1)}+C_{(1,0,0)}^{(0,0,2)} C_{(0,0,1)}^{(1,1,0)}+C_{(1,0,0)}^{(0,1,1)} \cdot\left(-C_{(0,0,1)}^{(1,0,1)}+C_{(0,1,0)}^{(1,1,0)}\right) \\
& =0 \in \mathrm{k}[\mathbf{C}] / \Re .
\end{aligned}
$$

We conclude that the origin is a singular point on $\mathbf{H}_{\mathrm{A}_{k}^{3}}^{4}$. It is known (see, for example, [Iarrobino 1985, Sec. 1, p. 147; Notari and Spreafico 2000, Sec. 5.1, p. 443]) that $\mathbf{H}_{\mathrm{A}_{k}^{3}}^{4}$ is irreducible and of dimension $3 \cdot 4=12$; it follows that the same is true of the open subscheme $U_{\beta}$. With a little more work, one can show that the 
$\mathrm{k}$-dimension of the tangent space at $p$ is equal to 18 (see, for example, [Miller and Sturmfels 2005, Ex. 18.31, p. 370]). Well-known examples such as this one (and Section 8.5) show that the nonsingularity and irreducibility of the Hilbert scheme of points of a nonsingular surface (first proved by Fogarty [1968]) do not carry over to $\mathbb{A}_{\mathrm{k}}^{n}$ for $n \geq 3$.

Connection to the Notari-Spreafico stratification. We continue for the moment to restrict $\mathrm{k}$ to an algebraically closed field. Before leaving this example, we note that the open subscheme $U_{\beta}$ under consideration can be obtained as the dense stratum in a stratification of Hilb $\mathbb{P}^{3}$ constructed by Notari and Spreafico [2000]. In general, they stratify $\mathrm{Hilb}_{\mathbb{P}_{\mathrm{k}}^{n}}^{p(z)}$ by locally closed subschemes of the form

$$
\left\{x \in \operatorname{Hilb}_{\mathbb{P}_{\mathrm{k}}^{n}}^{p(z)} \mid \operatorname{in}\left(I_{x}\right)=I_{0}\right\},
$$

where $x$ is a closed point, where $\operatorname{in}\left(I_{x}\right)$ denotes the initial ideal of the saturated ideal $I_{x}$ corresponding to $x$, and $I_{0}$ is a fixed monomial ideal. They treat the case of Hilb ${\mathbb{\mathbb { P } _ { k } ^ { 3 }}}_{\mathrm{k}}^{4}$ in [Notari and Spreafico 2000, Sec. 5.1, p. 443], writing as follows:

Using the rlex order, there is only one Borel-fixed, saturated monomial ideal with Hilbert function equal to $(1,4,4, \ldots)$ and it is the monomial ideal $I_{0}$ generated by $x_{0}^{2}, x_{0} x_{1}, x_{1}^{2}, x_{0} x_{2}, x_{1} x_{2}, x_{2}^{2}$. Following the construction of $\widetilde{V}_{I_{0}}$ [an affine scheme isomorphic to the stratum under construction in this case], we set

$$
\begin{aligned}
f_{1} & =x_{0}^{2}+a_{1,1} x_{0} x_{3}+a_{1,2} x_{1} x_{3}+a_{1,3} x_{2} x_{3}+a_{1,4} x_{3}^{2} \\
f_{2} & =x_{0} x_{1}+a_{2,1} x_{0} x_{3}+a_{2,2} x_{1} x_{3}+a_{2,3} x_{2} x_{3}+a_{2,4} x_{3}^{2} \\
& \vdots \\
f_{6} & =x_{2}^{2}+a_{6,1} x_{0} x_{3}+a_{6,2} x_{1} x_{3}+a_{6,3} x_{2} x_{3}+a_{6,4} x_{3}^{2}
\end{aligned}
$$

and, by imposing that $\left\{f_{1}, \ldots, f_{6}\right\}$ is a reduced Gröbner basis, we get the generators of an ideal $J$ in $\mathrm{k}\left[a_{1,1}, \ldots, a_{6,4}\right] \ldots$ The scheme $V$ [defined by $J$ ] has dimension 12 and its singularity is a dimension 3 subscheme, corresponding to the fat points ... Moreover, $V$ is irreducible because it is an open subscheme of the irreducible and generically smooth scheme Hilb ${\mathbb{\mathbb { P } _ { k } ^ { 3 }}}_{\mathrm{k}}^{4}$.

To "impose that $\left\{f_{1}, \ldots, f_{6}\right\}$ is a reduced Gröbner basis," we must require that the various $\mathrm{S}$-polynomials reduce to the zero polynomial modulo $\left\{f_{1}, \ldots, f_{6}\right\}$. For example, the S-polynomial involving $f_{1}$ and $f_{2}$ is

$$
\begin{aligned}
x_{1} \cdot f_{1}-x_{0} \cdot f_{2}= & x_{1} \cdot\left(a_{1,1} x_{0} x_{3}+a_{1,2} x_{1} x_{3}+a_{1,3} x_{2} x_{3}+a_{1,4} x_{3}^{2}\right) \\
& -x_{0} \cdot\left(a_{2,1} x_{0} x_{3}+a_{2,2} x_{1} x_{3}+a_{2,3} x_{2} x_{3}+a_{2,4} x_{3}^{2}\right) \\
= & a_{1,1} x_{3}\left(x_{0} x_{1}\right)+a_{1,2} x_{3}\left(x_{1}^{2}\right)+a_{1,3} x_{3}\left(x_{1} x_{2}\right)+a_{1,4} x_{1} x_{3}^{2} \\
& -a_{2,1} x_{3}\left(x_{0}^{2}\right)-a_{2,2} x_{3}\left(x_{0} x_{1}\right)-a_{2,3} x_{3}\left(x_{0} x_{2}\right)-a_{2,4} x_{0} x_{3}^{2} .
\end{aligned}
$$


To reduce this polynomial modulo the $f_{i}$, we add appropriate multiples of the $f_{i}$ to eliminate the "exposed terms", which are indicated by the parentheses in the last two displayed lines. That is, the expression

$$
\begin{aligned}
x_{1} \cdot f_{1}-x_{0} \cdot f_{2}+\left(a_{2,1} x_{3}\right) \cdot & f_{1}+\left(a_{2,2} x_{3}-a_{1,1} x_{3}\right) \cdot f_{2} \\
& -\left(a_{1,2} x_{3}\right) \cdot f_{3}+\left(a_{2,3} x_{3}\right) \cdot f_{4}-\left(a_{1,3} x_{3}\right) \cdot f_{5}+(0) \cdot f_{6}
\end{aligned}
$$

simplifies to a $\mathrm{k}\left[a_{i, j}\right]$-linear combination of the monomials $x_{0} x_{3}^{2}, x_{1} x_{3}^{2}, x_{2} x_{3}^{2}$, and $x_{3}^{3}$. The coefficients of this linear combination (and others similarly obtained) are then the generators of the ideal $J$. It should be clear that the de-homogenized version of the computation just outlined (set $x_{3}=1$ throughout) is identical to the construction of a pseudosyzygy and the corresponding coefficients $\rho_{\mathbf{j}}^{\left(\mathbf{b}_{1}, \mathbf{b}_{2}\right)}$ for the basis set $\beta$, with the variables indexed from 0 rather than 1 , and the indeterminates $C_{\mathbf{j}}^{\mathbf{b}}$ re-coded as $a_{i, j}$. Therefore, the variety $V$ is defined by an ideal that contains all the generators of the ideal cutting out $U_{\beta}$, and possibly more (since S-polynomials can be formed using all pairs of functions, not just the "adjacent" pairs); that is, $V$ is a closed subscheme of $U_{\beta}$. However, both $U_{\beta}$ and $V$ are irreducible of dimension 12 , hence equal. The other strata in this case will be lower-dimensional locally closed subschemes of $\mathrm{Hilb}_{\mathbb{P}_{k}^{3}}^{4}$.

Remark 39. The subscheme $U_{\beta} \subseteq \mathbf{H}_{\AA_{\mathrm{k}}^{n}}^{d}$ is not always a Notari-Spreafico stratum as just described. Indeed, they "prove that, on every irreducible component $\bar{H}$ of $\mathrm{Hilb}_{\mathbb{P}_{\mathrm{k}}^{r}}^{p(z)}$, the initial ideal corresponding to the element of the stratification that contains an open subset of $\bar{H}$ is Borel-fixed ..." [Notari and Spreafico 2000, p. 430]. Since $U_{\beta}$ is open in $\mathbf{H}_{\mathbb{A}_{k}^{n}}^{d}$, it cannot be a stratum unless the monomial ideal associated to $\beta$ is Borel-fixed.

8.5. Example 5. Let $n=4$, let $d=8$, and let

$$
\beta=\left\{1, x_{1}, x_{2}, x_{3}, x_{4}, x_{3}^{2}, x_{3} x_{4}, x_{4}^{2}\right\} .
$$

We again restrict $k$ to be an algebraically closed field. Iarrobino and Emsalem [1978] show that Hilb ${\mathbb{\mathbb { P } _ { k } ^ { 4 }}}^{8}$ is reducible, by exhibiting k-points $x \in \mathrm{Hilb}_{\mathbb{P}_{k}^{4}}^{8}$ at which the tangent space has dimension less than $4 \cdot 8=32$, the dimension of the principal component $\mathbf{H}_{\circ}^{d} \subseteq \mathbf{H}_{\AA_{\mathrm{k}}^{n}}^{d}$ (see Section 2.4). Here we observe that the same example shows that the open subscheme $U_{\beta}$ is reducible.

One can check that there are seven boundary monomials of degree 2:

$$
x_{1}^{2}, \quad x_{1} x_{2}, \quad x_{1} x_{3}, \quad x_{1} x_{4}, \quad x_{2}^{2}, \quad x_{2} x_{3}, \quad x_{2} x_{4},
$$

and ten boundary monomials of degree 3 :

$x_{1} x_{3}^{2}, \quad x_{2} x_{3}^{2}, \quad x_{3}^{3}, \quad x_{3}^{2} x_{4}, \quad x_{1} x_{3} x_{4}, \quad x_{2} x_{3} x_{4}, \quad x_{3} x_{4}^{2}, \quad x_{1} x_{4}^{2}, \quad x_{2} x_{4}^{2}, \quad x_{4}^{3}$. 
Therefore, there are $7 \cdot 3=21$ indeterminates $C_{\mathbf{j}^{\prime}}^{\mathbf{b}^{\prime}}$ such that both the boundary monomial $\mathbf{x}^{\mathbf{b}^{\prime}}$ and the basis monomial $\mathbf{x}^{\mathbf{j}^{\prime}}$ are of degree 2; we will call these indeterminates special.

Lemma 40. A special indeterminate $C_{\mathbf{j}^{\prime}}^{\mathbf{b}^{\prime}}$ cannot occur linearly in any of the polynomials $\rho_{\mathbf{j}}^{\left(\mathbf{b}_{1}, \mathbf{b}_{2}\right)}$. Furthermore, if $C_{\mathbf{j}^{\prime}}^{\mathbf{b}^{\prime}}$ occurs in a quadratic term $\pm C_{\mathbf{j}^{\prime}}^{\mathbf{b}^{\prime}} \cdot C_{\mathbf{j}^{*}}^{\mathbf{b}^{*}}$ of $\rho_{\mathbf{j}}^{\left(\mathbf{b}_{1}, \mathbf{b}_{2}\right)}$, then the other factor $C_{\mathbf{j}^{*}}^{\mathbf{b}^{*}}$ is not special.

Proof. By Remark 35, $C_{\mathbf{j}^{\prime}}^{\mathbf{b}^{\prime}}$ can occur linearly in $\rho_{\mathbf{j}}^{\left(\mathbf{b}_{1}, \mathbf{b}_{2}\right)}$ only if $\mathbf{b}^{\prime}=\mathbf{b}_{i}$ for $i=1$ or 2 , and $z_{i} \cdot \mathbf{x}^{\mathbf{j}^{\prime}}=\mathbf{x}^{\mathbf{j}}$. However, if we multiply any of the quadratic basis monomials $\mathbf{x}^{\mathbf{j}^{\prime}}$ by a variable, we land outside of the basis set; therefore, $z_{i}$ must equal \pm 1 (notation is that of Section 5.3). On the other hand, this means that $\mathbf{x}^{\mathbf{b}^{\prime}}=\mathbf{x}^{\mathbf{b}_{i}}$ is the least common multiple of the adjacent pair $\left(\mathbf{x}^{\mathbf{b}_{1}}, \mathbf{x}^{\mathbf{b}_{2}}\right)$, but this is impossible because $\mathbf{x}^{\mathbf{b}^{\prime}}$ is a boundary monomial of minimal degree 2 . Therefore, $C_{\mathbf{j}^{\prime}}^{\mathbf{b}^{\prime}}$ cannot occur linearly in $\rho_{\mathbf{j}}^{\left(\mathbf{b}_{1}, \mathbf{b}_{2}\right)}$.

Recall that $\rho_{\mathbf{j}}^{\left(\mathbf{b}_{1}, \mathbf{b}_{2}\right)}$ is the coefficient of $\mathbf{x}^{\mathbf{j}}$ in the dot product of the basic pseudosyzygy $P\left(\mathbf{b}_{1}, \mathbf{b}_{2}\right)$ and the tuple of functions $\left(G_{\mathbf{b}}\right)$ (see (30)). Consequently, there are two ways that $C_{\mathbf{j}^{\prime}}^{\mathbf{b}^{\prime}}$ can occur quadratically in $\rho_{\mathbf{j}}\left(\mathbf{b}_{1}, \mathbf{b}_{2}\right)$ :

- $C_{\mathbf{j}^{\prime}}^{\mathbf{b}^{\prime}}$ can occur in the $\mathbf{x}^{\mathbf{b}^{*}}$-component of $P\left(\mathbf{b}_{1}, \mathbf{b}_{2}\right)$, whence it multiplies $G_{\mathbf{b}^{*}}$ to yield the term $\pm C_{\mathbf{j}^{\prime}}^{\mathbf{b}^{\prime}} \cdot C_{\mathbf{j}}^{\mathbf{b}^{*}}$ in $\rho_{\mathbf{j}}^{\left(\mathbf{b}_{1}, \mathbf{b}_{2}\right)}$. In this case, $C_{\mathbf{j}^{\prime}}^{\mathbf{b}^{\prime}}=C_{\mathbf{j}^{\prime}}^{\mathbf{b}_{i}}$ (for $i=1$ or 2) is "exposed" when $G_{\mathbf{b}_{i}}$ is multiplied by $\pm z_{i}$, where $z_{i}$ is a variable. In turn, this implies that

$z_{i} \cdot \mathbf{x}^{\mathbf{j}^{\prime}}=\mathbf{x}^{\mathbf{b}^{*}}$ implies $\operatorname{deg}\left(\mathbf{x}^{\mathbf{b}^{*}}\right)=3$, which implies $C_{\mathbf{j}}^{\mathbf{b}^{*}}$ is not special.

- $C_{\mathbf{j}^{\prime}}^{\mathbf{b}^{\prime}}=C_{\mathbf{j}}^{\mathbf{b}^{\prime}}$ can occur as $(-1 \times)$ the coefficient of $\mathbf{x}^{\mathbf{j}}$ in the polynomial $G_{\mathbf{b}^{\prime}}$, which is then multiplied by $\pm C_{\mathbf{j}^{*}}^{\mathbf{b}^{*}}$ residing in the $\mathbf{x}^{\mathbf{b}^{\prime}}$-component of the basic pseudosyzygy $P\left(\mathbf{b}_{1}, \mathbf{b}_{2}\right)$ to yield the term $\pm C_{\mathbf{j}}^{\mathbf{b}^{\prime}} \cdot C_{\mathbf{j}^{*}}^{\mathbf{b}^{*}}$ in $\rho_{\mathbf{j}}^{\left(\mathbf{b}_{1}, \mathbf{b}_{2}\right)}$. In this case, $C_{\mathbf{j}^{*}}^{\mathbf{b}^{*}}=C_{\mathbf{j}^{*}}^{\mathbf{b}_{i}}($ for $i=1$ or 2$)$ is "exposed" when $G_{\mathbf{b}_{i}}$ is multiplied by $\pm z_{i}$, where $z_{i}$ is a variable. In turn, this implies that

$$
\mathbf{x}^{\mathbf{b}^{\prime}}=z_{i} \cdot \mathbf{x}^{\mathbf{j}^{*}} \text { implies } \operatorname{deg}\left(\mathbf{x}^{\mathbf{j}^{*}}\right)=1 \text {, which implies } C_{\mathbf{j}^{*}}^{\mathbf{b}^{*}} \text { is not special. }
$$

Since both possibilities imply that $C_{\mathbf{j}^{*}}^{\mathbf{b}^{*}}$ is not special, we are done.

Recall that $\mathbf{C}$ denotes the set of all the indeterminates $C_{\mathbf{j}}^{\mathbf{b}}$, and let $\mathbf{C}^{\prime}$ denote the subset of special indeterminates. Consider the surjection of polynomial rings

$$
\mathrm{k}[\mathbf{C}] \rightarrow \mathrm{k}\left[\mathbf{C}^{\prime}\right], \quad C_{\mathbf{j}}^{\mathbf{b}} \mapsto \begin{cases}C_{\mathbf{j}}^{\mathbf{b}} & \text { if } C_{\mathbf{j}}^{\mathbf{b}} \text { is special }, \\ 0 & \text { otherwise. }\end{cases}
$$

Corollary 41. The surjection (34) factors through the coordinate ring $\mathrm{k}[\mathbf{C}] / \Re$ of $U_{\beta}$, where $\Re$ is the ideal generated by the polynomials $\rho_{\mathbf{j}}^{\left(\mathbf{b}_{1}, \mathbf{b}_{2}\right)}$. Consequently, $U_{\beta}$ contains a closed subscheme $T$ isomorphic to 21-dimensional affine space, and 
whose k-points are obtained by assigning arbitrary scalars to the special indeterminates $C_{\mathbf{j}}^{\mathbf{b}}$, and 0 to the non-special $C_{\mathbf{j}}^{\mathbf{b}}$.

Proof. From Lemma 40, it is clear that if each non-special indeterminate is replaced with 0 , then the polynomials $\rho_{\mathbf{j}}^{\left(\mathbf{b}_{1}, \mathbf{b}_{2}\right)}$ all reduce to 0 .

Iarrobino and Emsalem [1978] prove that sufficiently general k-points in the closed subscheme $T$ have "small" tangent space as points of $\mathbf{H}_{\mathrm{A}^{4}}^{8}$; that is, the $\mathrm{k}$ dimension of the tangent space is less than $n \cdot d=4 \cdot 8=32$. They show this specifically for the point with coordinates

$$
\begin{aligned}
& C_{(0,0,2,0)}^{(2,0,0,0)}=-1, \quad C_{(0,0,0,2)}^{(1,1,0,0)}=-1, \\
& C_{(0,0,2,0)}^{(0,2,0,0)}=-1, \quad C_{(0,0,0,2)}^{(0,1,1,0)}=-1, \\
& C_{(0,0,1,1)}^{(1,0,1,0)}=-1 \text {, } \\
& C_{\mathbf{j}}^{\mathbf{b}}=0 \text { otherwise. }
\end{aligned}
$$

In fact, the k-dimension of the tangent space at this point is equal to $25=21+4$ : 21 is the dimension of $T$, and 4 is the number of degrees of freedom in $\mathbb{A}_{\mathrm{k}}^{4}$ in which the closed subschemes represented by the points of $T$ can be translated. (It is not hard to verify that all the subschemes of $\mathbb{A}_{\mathrm{k}}^{4}$ represented by points of $T$ are concentrated at the origin.) Using computer algebra, the author has confirmed that the (co)tangent space dimension at the displayed point is 25 by translating the scheme $U_{\beta}$ so that the point in question is at the origin, and then directly computing the k-dimension of (maximal ideal) $/(\text { maximal ideal })^{2}$. Since the dimension of $U_{\beta}$ is at least $4 \cdot 8=32$, by Corollary 9 , the presence of such points in $U_{\beta}$ implies that $U_{\beta}$ is reducible.

\section{Acknowledgements}

The author thanks the referee for many helpful questions and suggestions.

\section{References}

[Eisenbud 1995] D. Eisenbud, Commutative algebra, Graduate Texts in Mathematics 150, Springer, New York, 1995. MR 97a:13001 Zbl 0819.13001

[Eisenbud and Harris 2000] D. Eisenbud and J. Harris, The geometry of schemes, Graduate Texts in Mathematics 197, Springer, New York, 2000. MR 2001d:14002 Zbl 0960.14002

[Fogarty 1968] J. Fogarty, "Algebraic families on an algebraic surface", Amer. J. Math 90 (1968), 511-521. MR 38:5778 Zbl 0176.18401

[Fulton 1984] W. Fulton, Intersection theory, Ergebnisse der Mathematik und ihrer Grenzgebiete (3) 2, Springer, Berlin, 1984. MR 85k:14004 Zbl 0885.14002

[Grothendieck 1995] A. Grothendieck, "Techniques de construction et théorèmes d'existence en géométrie algébrique. IV. Les schémas de Hilbert”, pp. Exp. No. 221, 249-276 in Séminaire Bourbaki, Vol. 6, Soc. Math. France, Paris, 1995. MR 1611822 Zbl 0236.14003

[Grothendieck and Dieudonné 1971] A. Grothendieck and J. A. Dieudonné, Éléments de géométrie algébrique I, Grundlehren der mathematischen Wissenschaften 166, Springer, Berlin, 1971. MR 36:177a Zbl 0203.23301 
[Gustavsen et al. 2005] T. S. Gustavsen, D. Laksov, and R. M. Skjelnes, "An elementary, explicit proof of the existence of Hilbert schemes of points", 2005. math.AG/0506161

[Haiman 1998] M. Haiman, " $t, q$-Catalan numbers and the Hilbert scheme”, Discrete Math. 193:1-3 (1998), 201-224. MR 2000k:05264 Zbl 1061.05509

[Haiman and Sturmfels 2004] M. Haiman and B. Sturmfels, "Multigraded Hilbert schemes", J. Algebraic Geom. 13:4 (2004), 725-769. MR 2005d:14006 Zbl 02148302

[Hartshorne 1977] R. Hartshorne, Algebraic geometry, Springer, New York, 1977. MR 57:3116 Zbl 0367.14001

[Huibregtse 2002] M. E. Huibregtse, "A description of certain affine open subschemes that form an open covering of Hilb ${ }_{\mathbf{A}_{\mathrm{k}}^{2}}^{n}$ ”, Pacific J. Math. 204:1 (2002), 97-143. MR 2003h:14008 Zbl 1068.14007

[Iarrobino 1972] A. Iarrobino, Jr., "Reducibility of the families of 0-dimensional schemes on a variety”, Invent. Math. 15 (1972), 72-77. MR 46:170 Zbl 0227.14006

[Iarrobino 1985] A. Iarrobino, Jr., "Compressed algebras and components of the punctual Hilbert scheme", pp. 146-165 in Algebraic geometry, Sitges (Barcelona), 1983, Lecture Notes in Math. 1124, Springer, Berlin, 1985. MR 86k:14001 Zbl 0567.14001

[Iarrobino and Emsalem 1978] A. Iarrobino and J. Emsalem, "Some zero-dimensional generic singularities; finite algebras having small tangent space", Compositio Math. 36:2 (1978), 145-188. MR 81c:14004 Zbl 0393.14002

[Kollár 1996] J. Kollár, Rational curves on algebraic varieties, Ergebnisse der Mathematik und ihrer Grenzgebiete (3) 32, Springer, Berlin, 1996. MR 98c:14001 Zbl 0877.14012

[Miller and Sturmfels 2005] E. Miller and B. Sturmfels, Combinatorial commutative algebra, Graduate Texts in Mathematics 227, Springer, New York, 2005. MR 2110098 Zbl 1066.13001

[Mumford 1966] D. Mumford, Lectures on curves on an algebraic surface, Annals of Mathematics Studies 59, Princeton University Press, Princeton, 1966. MR 35:187 Zbl 0187.42701

[Notari and Spreafico 2000] R. Notari and M. L. Spreafico, "A stratification of Hilbert schemes by initial ideals and applications", Manuscripta Math. 101:4 (2000), 429-448. MR 2001b:14008 Zbl 0985.13006

[Sernesi 1986] E. Sernesi, Topics on families of projective schemes, Queen's Papers in Pure and Applied Mathematics 73, Queen's University, Kingston, ON, 1986. MR 88b:14006

[Skjelnes 2002] R. M. Skjelnes, "Resultants and the Hilbert scheme of points on the line", Ark. Mat. 40:1 (2002), 189-200. MR 2004g:14006 Zbl 1022.14003

Received June 20, 2004. Revised July 11, 2005.

MARK E. HUIBREgTSE

DePARTMENT OF MATHEMATics AND COMPUTER SCIENCE

SKIDMORE COLLEGE

SARATOGA SPRINGS

NEW YORK 12866

USA

mhuibreg@skidmore.edu

http://www.skidmore.edu/ mhuibreg/ 